\title{
Provenance and production technology of late medieval 'Besztercebánya/Banská Bystrica-type' high-quality stove tiles
}

\author{
Dorottya Györkös ${ }^{1,2,3}$ (D) Bernadett Bajnóczi ${ }^{2}$ (D) $\cdot$ György Szakmány ${ }^{3}$ (D) $\cdot$ Máté Szabó $^{2} \cdot$ Ralf Milke $^{4}$. \\ László Elöd Aradi $^{3}$ (D) Mária Tóth ${ }^{2}$
}

Received: 8 April 2020 / Accepted: 7 October 2020 / Published online: 28 November 2020

(C) The Author(s) 2020

\begin{abstract}
A unique collection of high-quality late medieval (fifteenth-sixteenth century) glazed and unglazed stove tiles from the northern part of the Carpathian Basin is of great interest to archaeologists and art historians. It is yet to be determined if these products, which are characterised by similar features, were produced in a single workshop, perhaps in Besztercebánya/Banská Bystrica (in present-day Slovakia), or in several workshops throughout the region. The first systematic multi-analytical investigation was carried out on the ceramic body and glaze of one hundred and seventeen tile fragments from six sites (Besztercebánya/Banská Bystrica, Fülek/Fil'akovo, and Csábrág/Čabrad' in Slovakia; Salgó, Eger, and Szécsény in Hungary) using polarising microscopy, $\mathrm{X}$-ray diffraction, electron microprobe, and Raman microspectroscopy analyses to determine the raw materials and production techniques used. Based on the petrographic characteristics, phase and chemical composition of the ceramic body, and the chemical composition and colourants of the glazes, the stove tiles can be classified into three primary groups. Tiles from different sites are different to each other, only the tiles from the Hungarian sites and from Fülek/Fil'akovo are similar. Thus, it is probable that the tiles were produced in several (at least three) workshops in the region from where they were then dispersed. The technological knowledge of the master(s) producing the polychrome Csábrág/Čabrad' tiles with tin-opacified glazes was higher than that of the master(s) producing the other tiles. However, the exact location of the workshops as well as their existence through time is still in unknown.
\end{abstract}

Keywords Stove tile $\cdot$ Late medieval $\cdot$ Lead glaze $\cdot$ Provenance $\cdot$ Production technology

Dorottya Györkös

dorkagyorkos@gmail.com

Bernadett Bajnóczi

bajnoczi.bernadett@csfk.mta.hu

1 Isotope Climatology and Environmental Research Centre, Institute for Nuclear Research, Bem tér 18/c, Debrecen H-4026, Hungary

2 Institute for Geological and Geochemical Research, Research Centre for Astronomy and Earth Sciences, Budaörsi út 45, Budapest H-1112, Hungary

3 Department of Petrology and Geochemistry, Eötvös Loránd University, Pázmány Péter sétány 1/C, Budapest H-1117, Hungary

4 Institute of Geological Sciences, Freie Universität, Malteserstr 74-100, D-12249 Berlin, Germany

\section{Introduction}

After the Roman hypocaustrum system, only ovens and open fireplaces were used for heating, until stoves were invented around the twelfth century in the region of Switzerland (de Langhe et al. 2015; Kocsis 2018). The first stoves in medieval Hungary were initially used in the royal court, and then by various other social classes from the fifteenth century. Stoves were the beating heart of homes from peasant houses to castles, and made winters a lot more bearable. The greatest benefit of the stoves is that it does not produce smoke inside the homes. Stoves were built up from clay plasters that were complemented with stove tiles to expand the heating surface (Roth-Heege 2018). Nevertheless, stoves were not only used for heating but also as an important symbol of the owner's wealth and the fashion of the time. Thus, from the end of the fourteenth century, the stoves were decorated with diverse ornamentations, such as ornamental motifs, coats of arms, 
and motifs or scenes from the Bible. Due to the wide range of decorative elements unique to a given time period, archaeologists and art historians can typify the characteristic symbols and scenes illustrated on the front of the tiles. At the end of the fourteenth century, coloured glazes were a new decorative element on high-quality stove tiles, and at the end of the fifteenth century, polychromy (the art of decorating a tile with various colours) became common. With the spread of quantity production, the use of negatives (created by a master or from the motifs on a standing stove) had begun (Kocsis et al. 2003; Kocsis 2018). Stove tiles are therefore one of the most important tools to investigate contemporary art, social and commercial networks, and technological advancements.

Despite the wide range of preserved stove tiles (e.g. in royal palaces, castles, noble mansions, cloisters, and town houses) and their prevalence among the archaeological assemblages, they garnered only limited attention from an archaeometric point of view. Thus far, only a few archaeometric studies have dealt with medieval and early modern stove tiles, such as Ernée et al. (2004); Vecstaudža et al. (2013); de Langhe et al. (2015); Kreiter and Pánczél (2016); Maggetti et al. (2014); Maggetti (2016, 2019); Maggetti and Serneels (2016); and Bauer et al. in press. Additionally, comprehensive archaeometric studies on stove tiles from the Carpathian Basin still lack detailed microstructural, chemical, and phase analyses of the ceramic body and the glaze. Thorough studies are required to determine the raw material, the maximum firing temperature, and the type of glazes and their colourants, so that the production technology, as well as the products of the different centuries, regions, and workshops/masters, may be determined.

At the end of the nineteenth century, decorative glazed stove tiles, waste fragments, and negatives (moulds) were discovered in the city of Besztercebánya (Banská Bystrica, present-day Slovakia), in the courtyard of the 'Bothár' house. Over 10 years later, in the nearby 'Ébner' house, similar glazed tile fragments were found in a secondary position (S. Cserey, 1974; Parádi 1984) (Fig. 1). The importance of the findings is not solely the unique, high-quality nature of the tiles, but also the fact that a stove tile from the 'Ébner' house perfectly fits into one of the moulds unearthed from a pit at the 'Bothár' house (Fig. 1c, d). The archaeological and art historical investigations showed that the stove tiles belong to a special late medieval (fifteenth-sixteenth centuries) collection that spanned in the northern part of the Carpathian Basin and its surrounding areas (Fig. 2). These tiles illustrate both religious (e.g. portraits of saints and prophets) and profane (e.g. the scenes of Aristoteles and Phyllis) motifs (Gruia 2007, 2013). The circumstantial motifs and the illustrated topics are exceptional in the region in this period. In total, over 200 fragments of stove tiles from the northern part of medieval Hungary (present-day Hungary, Slovakia, and Czech Republic) can be associated with this collection (Bodnár 1988; Mácelová 2005, 2006, 2009; László 2012;
Kvietok and Mácelová 2013; Mordovin 2015; Rakonczay 2018, 2020; Anderko in press). The unique, ornamental stoves were ordered by the nobility; therefore, most of the artefacts were found in castles or fortresses. However, some of them were excavated in the cities of Besztercebánya/Banská Bystrica (present-day Slovakia) and Szécsény (Hungary). In Besztercebánya/Banská Bystrica, the tiles were found throughout the medieval city and, in several cases, were discovered among waste fragments which suggests that a local production centre existed somewhere in the city (Hoššo 2005; Mezei 2013, 2016). Although contemporaneous written sources are scarce, combined with detailed investigations of the motifs, they lead archaeological and art historical researchers to suggest that these stove tiles were manufactured between the second half of the fifteenth century to the first half of the sixteenth century (Balogh-László et al. 2018). The precise motifs of the tiles can be connected to wood carvers, who worked in the region at this time on the altarpiece of the parish church of Besztercebánya/ Banská Bystrica (Mezei 2016). It is proposed that they created the wooden moulds that later became ceramic moulds to be used for the decoration of the tiles. The small number of findings, as well as the iconographical similarities, suggests a local trend. However, there are differences between the elaboration of the motifs found at each site. Lack of contemporaneous written evidence and dated archaeological context makes it difficult to determine if these products were made in workshop(s) situated in Besztercebánya/Banská Bystrica or in several workshops in the region that influenced each other.

Paralleling with the ongoing archaeological and art historical studies, the primary goal of this first systematic archaeometric research is to determine the raw materials and production technology of the ceramic body, as well as of the glaze of the stove tiles from various archaeological sites using a multi-analytical methodology. Through examination of similarities and differences in the applied materials and technology, we endeavour to make a distinction between the products of the masters and to determine the number of possible workshops.

Petrographic characteristics and the phase composition of the ceramic body were already analysed in detail (Györkös et al. 2018, in press). In this paper, we present a comprehensive analysis of the late medieval stove tiles, focusing on the chemical composition of the ceramic body, as well as the microstructural and microchemical characterisation of the glazes.

\section{Materials}

One hundred and seventeen tile samples were examined from six locations of the northern part of the Carpathian Basin and its surrounding areas (see Online Resource 1). The investigated sites are the two aforementioned houses in the city of 
Fig. 1 Late medieval stove tiles from Besztercebánya/Banská Bystrica a Adam and Eve, Ébner house b Portrait of a Bearded Man, Ébner house. Stove tile and negative designed by scales $\mathbf{c}$ BB 52.3757.1, Bothár house, d BB_52.3758.1, Ébner house (Museum of Applied Arts, Budapest, processed by Emese Balogh-László)

\section{a}

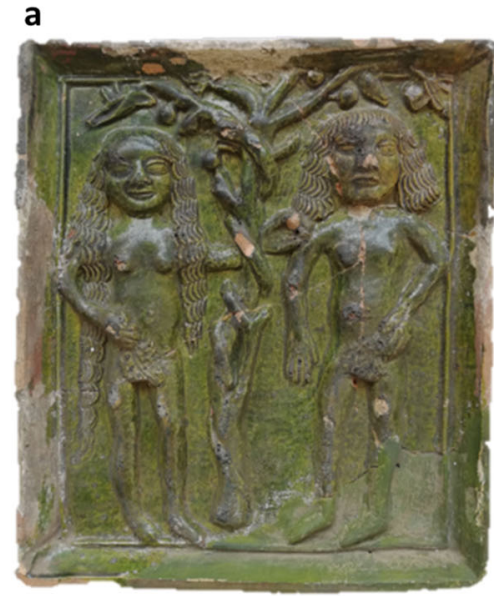

b

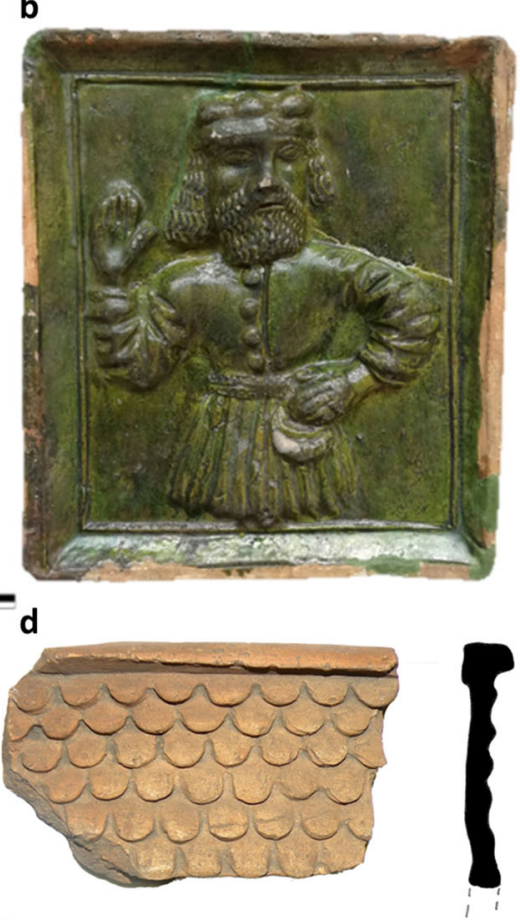

Besztercebánya/Banská Bystrica (21 pieces, N $48^{\circ} 44^{\prime} 3.85^{\prime \prime}$, E $19^{\circ} 8^{\prime} 37.89^{\prime \prime}{ }^{1}$ _Bothár' house and N 48 44' 7.21", E $19^{\circ}$ 8' 39.59"_ 'Ébner' house), as well as the castles of Csábrág/ Čabrad' (12 pieces, N $48^{\circ} 14^{\prime} 38.59^{\prime \prime}$, E $19^{\circ} 6^{\prime} 22.62^{\prime \prime}$ ) and Fülek/Fil'akovo (10 pieces, N 48 $16^{\prime} 17.48^{\prime \prime}$, E $19^{\circ} 49^{\prime}$ $\left.30.45^{\prime \prime}\right)$ from present-day Slovakia. In present-day Hungary, the analysed tiles were excavated in the fortress of Salgó (50 pieces, $\mathrm{N} 48^{\circ} 8^{\prime} 41.04^{\prime \prime}$, E $19^{\circ} 50^{\prime} 49.14^{\prime \prime}$ ) and the castle of Eger (13 pieces, N $47^{\circ} 54^{\prime} 14.30^{\prime \prime}$, E $20^{\circ} 22^{\prime} 47.52^{\prime \prime}$ ), as well as in the city of Szécsény (11 pieces, N 48 $4^{\prime} 55.65^{\prime \prime}$, E $19^{\circ}$ $31^{\prime} 11.02^{\prime \prime}$ and N 48 4' 56.90", E $19^{\circ} 31^{\prime} 15.55^{\prime \prime}$ ) (Fig. 2). With the exception of Csábrág/Čabrad' (Rakonczay 2018, 2020) and Salgó (László 2012; Feld et al. 2013), the tiles cannot be connected to a precise archaeological context, because they were found scattered throughout the archaeological sites. The analysed samples belong to the Museum of Applied Arts (Budapest, Hungary), the Hungarian National Museum (Budapest, Hungary), the István Dobó Castle Museum (Eger, Hungary), the Ferenc Kubinyi Castle Museum (Szécsény, Hungary), the Slovak National Museum (Bratislava, Slovakia), and the Institute of Archaeology of the Slovak Academy of Sciences (Nitra, Slovakia).

Macroscopically, the ceramic bodies of the stove tiles from Hungary and Fülek/Fil'akovo are white or, rather, light pink, in which white, grey, and black grains occur. In contrast, the body of the tiles from Besztercebánya/Banská Bystrica and Csábrág/Čabrad' are reddish and sparsely contain white, red,

${ }^{1}$ The coordinates of the archaeological sites are provided by Google Earth and grey grains. Two-thirds of the assemblage (79 pieces) is glazed. Various tones of green glaze dominate, but yellow, and rarely white, blue, and brown glaze also appear (Fig. 3).

\section{Methods}

Petrographic analysis of the ceramic body of all the stove tiles was conducted with a Nikon Eclipse E600 polarising microscope to determine texture, non-plastic components, and raw materials used. Photomicrographs and the grain size of the particles were recorded by the SPOT software (v4.6.4.2). The number of inclusions and the degree of sorting and roundness of the non-plastic components were determined in accordance with the guidelines of the Prehistoric Ceramics Research Group (PCRG 2010). The guidelines in Adams et al. (1984) were considered for the size categories.

Characteristic mineral assemblages and accessories of the ceramic bodies, as well as microstructure, inclusions, and chemical composition of the glazes, were studied on carboncoated, polished thin sections and cross sections using a JEOL Superprobe-733 electron microprobe (operating conditions: $20-\mathrm{kV}$ acceleration voltage, 6-nA beam current). The instrument was equipped with an Oxford Instruments INCA Energy 2000 and an Oxford Instruments AZtec X-ACT Premium SDD type energy-dispersive X-ray spectrometers (EDS), respectively. For the glazes, well-known artificial glasses (NMNH 117218-4, -1, -2, -3, i.e. Corning archaeological reference glasses A, B, C, and D, Adlington 2017), provided by 
the Department of Mineral Sciences, Smithsonian Institution (Washington, DC, USA), were used as reference materials for the main elements. Furthermore, $\mathrm{SnO}_{2}$ for $\mathrm{Sn}$, pure metal for $\mathrm{Co}$, chalcopyrite for $\mathrm{Cu}$, and antimony telluride for $\mathrm{Sb}$ were applied (provided by the Taylor Co., Stanford, CA, USA). This method, however, cannot distinguish between oxidation states of polyvalent elements; thus, all iron is expressed as $\mathrm{FeO}$ and all manganese as $\mathrm{MnO}$. $\mathrm{Cl}$ was also measured but fell below the detection limit for all samples. Typical detection limits are $<0.2 \%$ for $\mathrm{Mg}, \mathrm{Al}, \mathrm{Si}, \mathrm{P}, \mathrm{S}, \mathrm{Cl}, \mathrm{Ti}, \mathrm{Mn}, \mathrm{Fe}, \mathrm{Cu}, \mathrm{Ba}$, and $\mathrm{Pb}$ and $<0.5 \%$ for $\mathrm{Na}, \mathrm{K}$, and $\mathrm{Ca}$, respectively. For the accuracy and precision of the EDS measurements, see Online Resource 2. The chemical composition of the glaze was determined using area measurements (areas of up to $150 \times$ $150 \mu \mathrm{m}, 100$-s acquisition time) including colourants and avoiding crystals at the ceramic body-glaze interface, fractures, pores, and weathered areas. At least three area measurements were performed on each glaze. From the ceramic bodyglaze interface to the outer edge of the glaze, spot and line measurements were taken (40- and 60-s acquisition time, respectively) to determine if elements transferred between the ceramic body and the glaze, thus indicating whether single or double firing was used. The analytical totals of the glaze vary in the range of 98 and $102 \mathrm{wt} \%$. Area and spot measurements were averaged. Additionally, spot measurements (40-s acquisition time) were performed on the newly formed crystals at the body-glaze interface and in the glaze. During the EDS analysis of these crystals and the analysed components of the ceramic body, natural and artificial materials provided by the Taylor Co. (Stanford, CA, USA) were used as reference materials.

The quantitative chemical composition of the matrix of the selected ceramic bodies (15 samples) was determined on carbon-coated, polished thin sections using a JEOL JXA-8200 Superprobe to determine the raw materials. The instrument was equipped with WDS spectrometers and operated at 15kv accelerating voltage, 20-nA beam current using a beam diameter of $20 \mu \mathrm{m}$. The used reference materials were $\mathrm{P}$ apatite, $\mathrm{Na}$ - andesine, $\mathrm{Fe}$ - olivine, $\mathrm{Mn}$ - pure metal, $\mathrm{Mg}$ olivine, $\mathrm{K}$-sanidine, $\mathrm{Ca}$ - andesine, $\mathrm{Si}$ - sillimanite, and $\mathrm{Ti}$ - pure metal. For each sample, 100 spots were measured in a $2000 \times 2000-\mu \mathrm{m}$ area. Analyses referring to the matrix

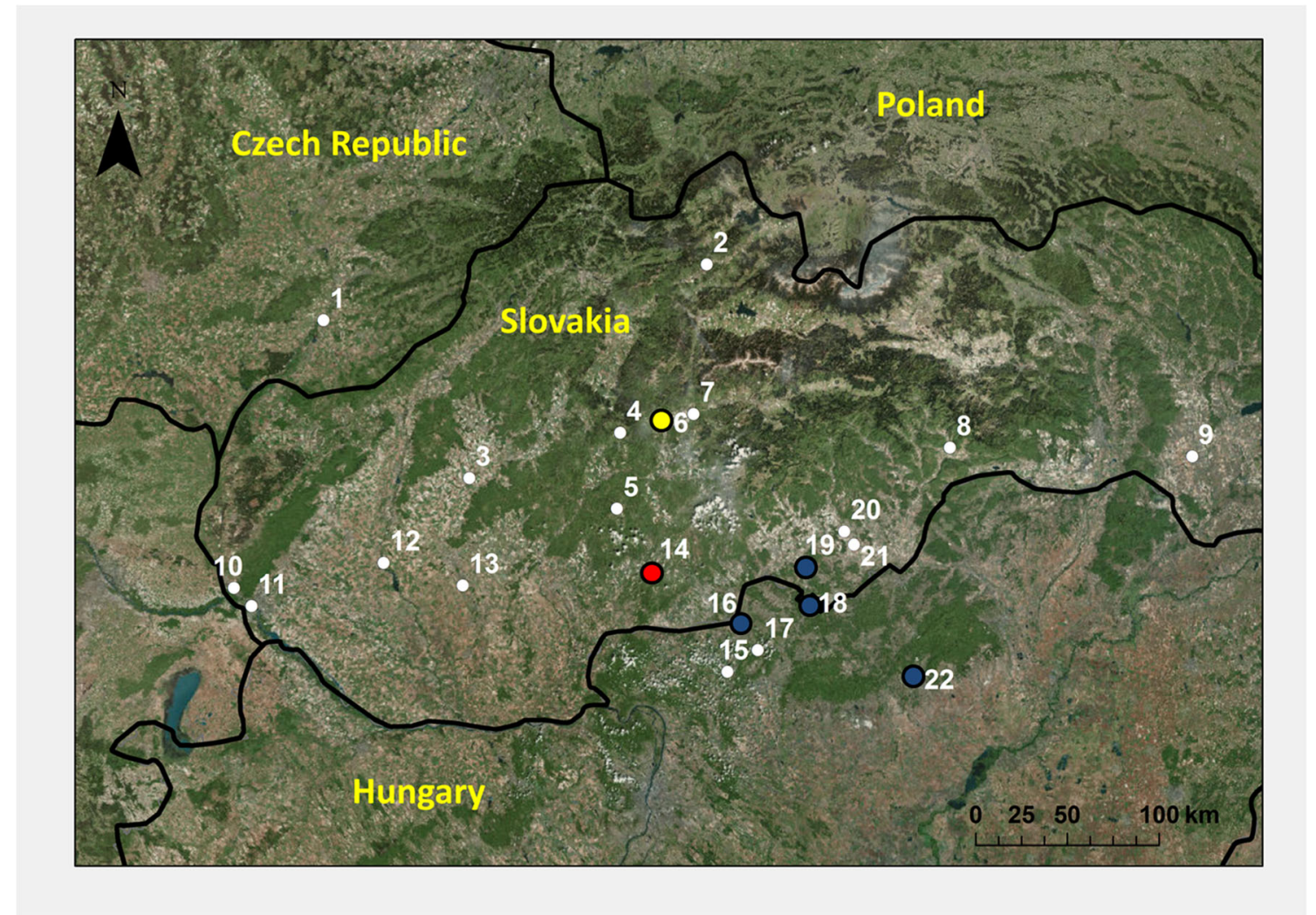

Fig. 2 Map showing the occurrences of the 'Besztercebánya/Banská Bystrica-type' stove tiles in the northern part of the Carpathian Basin and its surroundings (data collected by Emese Balogh-László). Investigated sites are highlighted by coloured dots (yellow dot - group 1, blue dots - group 2, red dot-group 3). 1: Magyarhradis/Uherské Hradiště, 2: Árva/Oravsky hrad, 3: Kistapolcsány/Topolčianky, 4: Körmöcbánya/Kremnica, 5: Selmecbánya/Banská Štiavnica, 6:
Besztercebánya/Banská Bystrica, 7: Zólyomlipcse/Slovenská L’upča, 8: Rozsnyó/Rožňava, 9: Tőketerebes/Trebišov, 10: Dévény/Devín, 11: Pozsony/Bratislava, 12: Sempte/Šintava, 13: Berencs/Branč, 14: Csábrág/Čabrad’, 15: Szanda, 16: Szécsény, 17: Hollókő, 18: Salgó, 19: Fülek/Fil'akovo, 20: Rimaszombat-Barátkút/Rimavská Sobota, 21: Jánosi/Rimavské Janovce, 22: Eger 
Fig. 3 Typical glazes of the examined stove tiles. a Eger, E_2 (yellow and green). b Salgó, S 2012.1.83.3 (green). c Csábrág/ Čabrad', Cs_2016.89.3 (white, blue and yellow). d Fülek/ Filakovo, F_2.589 (green, light brownish-white, brown). e Fülek/ Fil'akovo, F_1.602 (yellow and green). f Csábrág/Čabrad', Cs_ 2016.211.1 (dark green)
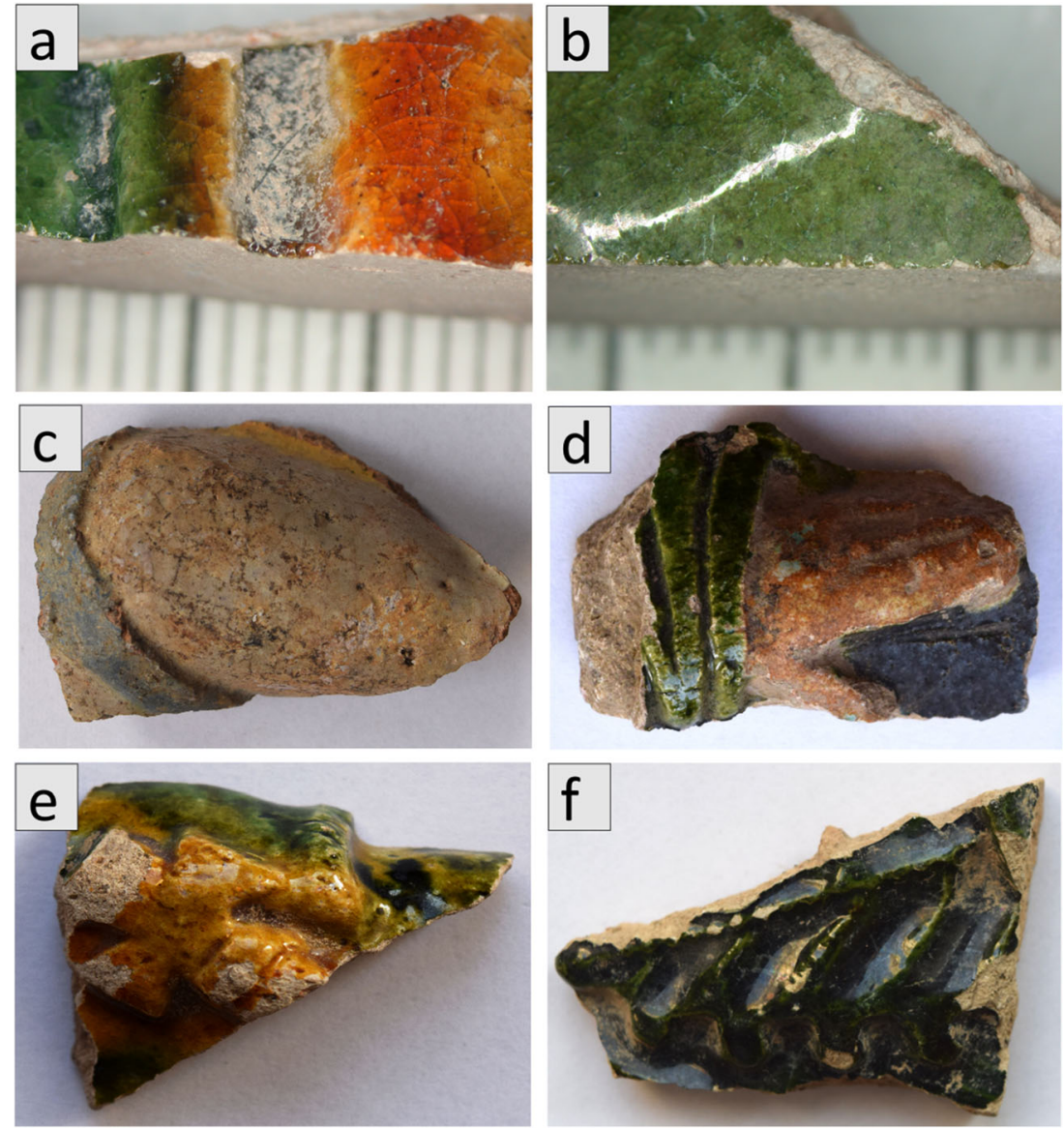

(excluding quartz and calcite) were selected for evaluation. The analytical totals of the matrix varied between 73.5 and $86.5 \mathrm{wt} \%$ because the ceramic body was porous. Totals were normalised to $100 \mathrm{wt} \%$ and averaged.

Phase composition of all ceramic bodies was determined on powdered samples by X-ray diffraction analysis to estimate the firing temperature of the tiles. Most of the samples were analysed using a Philips PW 1730 diffractometer with BraggBrentano alignment. Instrumental parameters were $\mathrm{CuK}_{\alpha}$ radiation, $45-\mathrm{kV}$ tension, 35-mA intensity, 0.05-0.01 ${ }^{\circ} 2 \Theta$ step size, 1 -s time constant, $1^{\circ}$ detector slit, $1^{\circ}$ divergence slit, PW1050/25 goniometer, graphite monochromator, and proportional counter detector. Samples from Fülek/Fil'akovo and Csábrág/Čabrad' were measured with a RIGAKU MINIFLEX 600 diffractometer with Bragg-Brentano alignment. Instrumental parameters were $\mathrm{CuK}_{\alpha}$ radiation, $40-\mathrm{kV}$ tension, $15-\mathrm{mA}$ intensity, $0.05^{\circ} 2 \Theta$ step size, $1^{\circ}$ detector slit, $1^{\circ}$ divergence slit, and graphite monochromator.

Raman microspectroscopy analysis was performed to identify crystals and colourants in the glazes of 13 stove tiles. A confocal HORIBA Labram HR (high resolution) 600 spectrometer with Nd:YAG laser $(\lambda=532 \mathrm{~nm})$ excitation, 1800gr/mm optical grating, a 50-200 $\mu \mathrm{m}$ confocal hole, 2-100-s acquisition time, and $\mathrm{a} \times 100$ objective $(\mathrm{NA}=0.9)$ was employed. The laser spot size (lateral) was measured to be
$1 \mu \mathrm{m}$, and the depth resolution was $\sim 1.5 \mu \mathrm{m}$ (using a $50-\mu \mathrm{m}$ confocal hole and $\times 100$ objective). The laser power was $130 \mathrm{~mW}$ at the source and $\sim 25 \mathrm{~mW}$ at the sample surface, which was occasionally reduced to $\sim 0.25-13 \mathrm{~mW}$ with optical filters to avoid photo-oxidation and heating of the samples. The spectral resolution was $3.0 \mathrm{~cm}^{-1}$ at $1398.5 \mathrm{~cm}^{-1}$ (full width at half maximum of one neon line). Data evaluation (background fitting and peak fitting using the GaussianLorentzian sum profiles) was performed using LabSpec 5 software. The mineral phases were identified based on their most intensive Raman bands, using the RRUFF database (rruff.info; Lafuente et al. 2015) as a guide.

\section{Results}

\section{Ceramic body}

The ceramic body was investigated by polarising microscopy and X-ray diffraction analysis for all the studied tiles. The petrographic characteristics (texture, grain size, type, and quantity of the typical non-plastic components) aid in the classification of the ceramic body of the analysed stove tiles into three primary groups (Fig. 4). Detailed descriptions of the 
Fig. 4 Polarising microscope images of the ceramic body of the studied stove tiles. a Group 1BB_Adam and Eve,

Besztercebánya/Banská Bystrica

(PPL). b Group 2-S

2012.1.83.3, Salgó (XP). c Group 2-S_2012.1.83.7, Salgó (XP). d Group 2-SZ Ornamental,

Szécsény (XP). e Group 3-CS 2016.89.4, Csábrág/Čabrad' (PPL). f E_Green-glazed grape tendrils 7, Eger (XP). ARF, argillaceous rock fragment; crf, carbonate rock fragment; kfs, Kfeldspar; pq, polycrystalline quartz; vrf, volcanic rock fragment; q, quartz
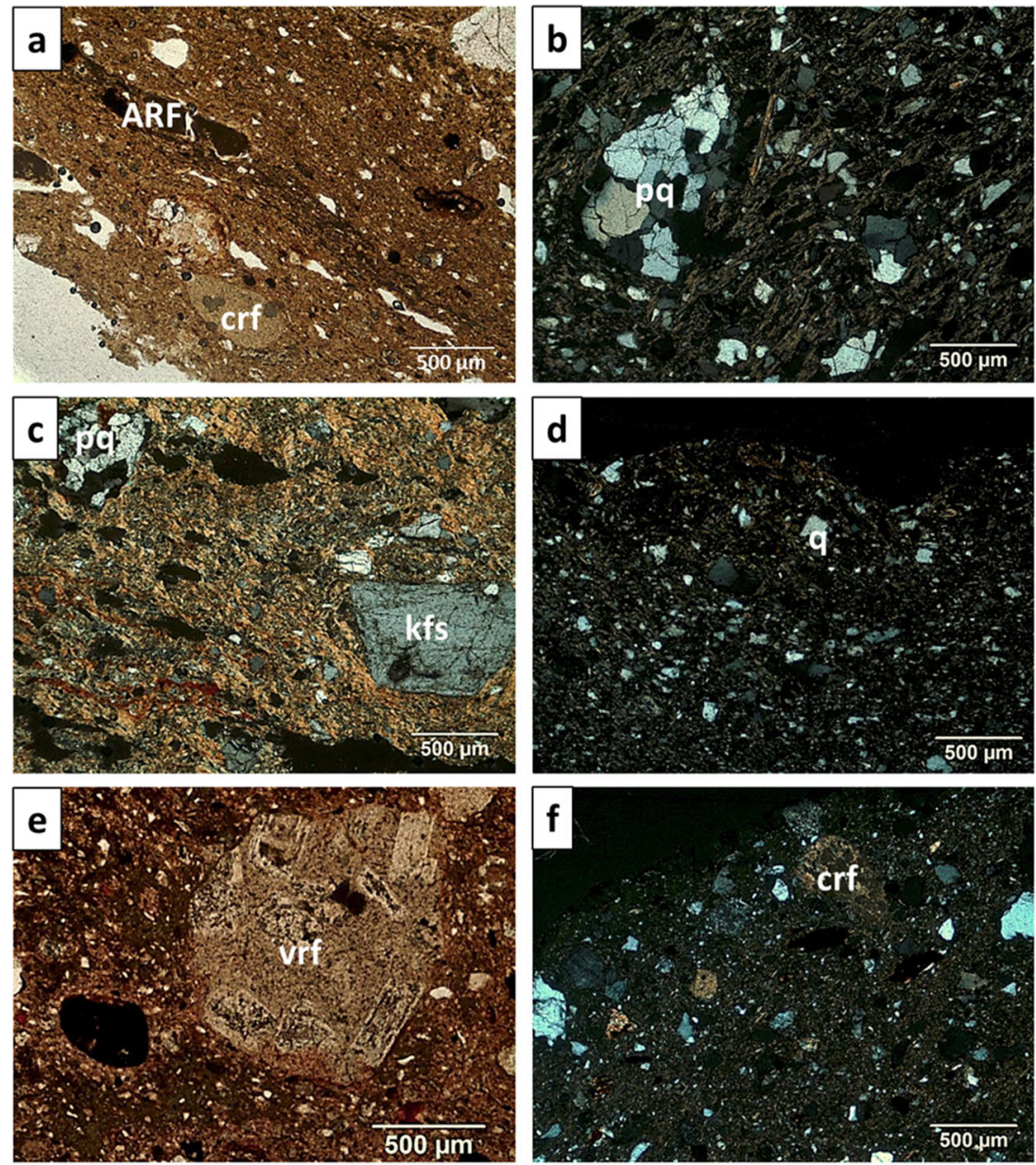

groups and subgroups were published by Györkös et al. (2018, in press). Here we provide a brief summary.

In addition to the medium silt to very fine sand-sized quartz, K-feldspar, plagioclase, micas (biotite, muscovite), calcite, and opaque minerals, the ceramic body of group 1 contains medium and coarse sand-sized sub-angular carbonate rock fragments, granitoid rock fragments, quartzite, and a single basic rock fragment. The percentage of carbonatic rock fragments varies between 1 and 15\%. In some samples, argillaceous rock fragments (ARF) also occur. All the examined tiles from Besztercebánya/Banská Bystrica form this group.

In contrast, samples from Northern Hungary and Fülek/ Fil'akovo comprise group 2 and lack carbonatic rock fragments. Grain sizes of the inclusions vary between medium silt and coarse sand. Mono- and polycrystalline quartz, K-feldspar, plagioclase, microline, micas, and opaque minerals occur in the medium silt to very fine and medium sand grain size fractions. Additionally, most of the tiles (subgroup 2A) contain large amounts (up to 35\%) of coarse sand-sized inclusions, with trimodal grain size distribution. Characteristic non-plastic inclusions are granitoid and micaceous quartzite fragments. Two independent subgroups (subgroups 2B and $2 \mathrm{C}$ ) consist of tile bodies with unimodal grain size distribution (medium silt to medium sand-sized inclusions) and different amounts of quartz, plagioclase, and K-feldspar grains. Additionally, mullite detected by XRD is present in some bodies of subgroup 2A and in all bodies of subgroup 2B. Group 3 is distinguished from the other two groups based on the appearance of coarse to very coarse sand-sized, hydrothermally altered volcanic rock fragments (1 to $10 \%$ ). These peculiar inclusions occur only in the ceramic body of the tiles from Csábrág/Čabrad'.

Accessory minerals are mainly ilmenite, titanium oxide, and zircon in all groups. In group 1 apatite, monacite and, in one sample, barite (connected to a granitoid rock fragment) were detected.

One tile from Eger (E_Green-glazed grape tendrils 7) is an outlier; however, it is similar to group 1 in the appearance of carbonates (calcite grains and micritic limestone fragments) in the body. Quartz, K-feldspar, plagioclase, altered micas (biotite), opaque minerals, and quartzite and limonitic fragments are present in bimodal grain size distribution.

Quantitative chemical analysis of the matrix of 15 ceramic bodies (representative samples for all petrographic groups and subgroups) (Table 1) reveals that small chemical differences occur between the tiles from different sites (Fig. 5). 
Table 1 Chemical composition of the matrix of the ceramic body of selected tiles from each petrographic group (in wt $\%$, normalised to $100 \%$, average and standard deviation (in parenthesis) of spot measurements performed by EPMA-WDS analysis. $n$ number of measurements, $B B$ Besztercebánya/Banská Bystrica, CS Csábrág/Čabrad', $E$ Eger, $F$ Fülek/ Fil’akovo, $S$ Salgó, $S Z$ Szécsény)

\begin{tabular}{|c|c|c|c|c|c|c|c|c|c|c|c|c|}
\hline & Sample & $n$ & $\mathrm{SiO}_{2}$ & $\mathrm{Al}_{2} \mathrm{O}_{3}$ & $\mathrm{TiO}_{2}$ & $\mathrm{FeO}$ & $\mathrm{MnO}$ & $\mathrm{MgO}$ & $\mathrm{CaO}$ & $\mathrm{Na}_{2} \mathrm{O}$ & $\mathrm{K}_{2} \mathrm{O}$ & $\mathrm{P}_{2} \mathrm{O}_{5}$ \\
\hline \multirow[t]{3}{*}{ Group 1} & BB_Adam and Eve & 44 & $\begin{array}{l}66.03 \\
(3.22)\end{array}$ & $\begin{array}{l}22.46 \\
(2.48)\end{array}$ & $\begin{array}{l}0.75 \\
(0.59)\end{array}$ & $\begin{array}{l}3.75 \\
(1.02)\end{array}$ & $\begin{array}{l}0.02 \\
(0.03)\end{array}$ & $\begin{array}{l}1.74 \\
(0.33)\end{array}$ & $\begin{array}{l}0.81 \\
(0.27)\end{array}$ & $\begin{array}{l}0.51 \\
(0.63)\end{array}$ & $\begin{array}{l}3.70 \\
(1.02)\end{array}$ & $\begin{array}{l}0.22 \\
(0.32)\end{array}$ \\
\hline & BB_St. Peter & 45 & $\begin{array}{l}66.59 \\
(2.96)\end{array}$ & $\begin{array}{l}21.36 \\
(2.24)\end{array}$ & $\begin{array}{l}0.74 \\
(0.57)\end{array}$ & $\begin{array}{l}3.61 \\
(1.15)\end{array}$ & $\begin{array}{l}0.06 \\
(0.23)\end{array}$ & $\begin{array}{l}2.29 \\
(0.39)\end{array}$ & $\begin{array}{l}0.83 \\
(0.21)\end{array}$ & $\begin{array}{l}0.37 \\
(0.13)\end{array}$ & $\begin{array}{l}4.05 \\
(1.15)\end{array}$ & $\begin{array}{l}0.09 \\
(0.03)\end{array}$ \\
\hline & BB_52.3757.1 & 39 & $\begin{array}{l}64.19 \\
(2.80)\end{array}$ & $\begin{array}{l}20.73 \\
(1.93)\end{array}$ & $\begin{array}{l}0.81 \\
(0.41)\end{array}$ & $\begin{array}{l}3.50 \\
(0.89)\end{array}$ & $\begin{array}{l}0.03 \\
(0.03)\end{array}$ & $\begin{array}{l}3.95 \\
(0.92)\end{array}$ & $\begin{array}{l}2.29 \\
(1.06)\end{array}$ & $\begin{array}{l}0.31 \\
(0.10)\end{array}$ & $\begin{array}{l}3.87 \\
(1.97)\end{array}$ & $\begin{array}{l}0.31 \\
(0.08)\end{array}$ \\
\hline \multirow[t]{8}{*}{ Group 2} & SZ_Ornamental & 25 & $\begin{array}{l}63.59 \\
(3.16)\end{array}$ & $\begin{array}{l}28.21 \\
(2.67)\end{array}$ & $\begin{array}{l}0.94 \\
(0.38)\end{array}$ & $\begin{array}{l}1.66 \\
(0.24)\end{array}$ & $\begin{array}{l}0.02 \\
(0.02)\end{array}$ & $\begin{array}{l}0.83 \\
(0.16)\end{array}$ & $\begin{array}{l}1.32 \\
(0.31)\end{array}$ & $\begin{array}{l}0.48 \\
(0.16)\end{array}$ & $\begin{array}{l}2.85 \\
(0.36)\end{array}$ & $\begin{array}{l}0.10 \\
(0.03)\end{array}$ \\
\hline & SZ_Coat of arms 1. & 17 & $\begin{array}{l}68.30 \\
(3.71)\end{array}$ & $\begin{array}{l}24.72 \\
(2.90)\end{array}$ & $\begin{array}{l}1.30 \\
(1.28)\end{array}$ & $\begin{array}{l}1.59 \\
(0.31)\end{array}$ & $\begin{array}{l}0.01 \\
(0.01)\end{array}$ & $\begin{array}{l}0.63 \\
(0.13)\end{array}$ & $\begin{array}{l}0.39 \\
(0.05)\end{array}$ & $\begin{array}{l}0.43 \\
(0.09)\end{array}$ & $\begin{array}{l}2.60 \\
(0.34)\end{array}$ & $\begin{array}{l}0.04 \\
(0.04)\end{array}$ \\
\hline & S_2012.1.83.1 & 52 & $\begin{array}{l}57.11 \\
(2.47)\end{array}$ & $\begin{array}{l}33.70 \\
(2.01)\end{array}$ & $\begin{array}{l}0.95 \\
(0.65)\end{array}$ & $\begin{array}{l}2.36 \\
(0.32)\end{array}$ & $\begin{array}{l}0.03 \\
(0.02)\end{array}$ & $\begin{array}{l}0.96 \\
(0.25)\end{array}$ & $\begin{array}{l}1.04 \\
(0.18)\end{array}$ & $\begin{array}{l}0.29 \\
(0.08)\end{array}$ & $\begin{array}{l}3.38 \\
(1.54)\end{array}$ & $\begin{array}{l}0.17 \\
(0.05)\end{array}$ \\
\hline & S_S6 & 33 & $\begin{array}{l}58.66 \\
(2.91)\end{array}$ & $\begin{array}{l}32.80 \\
(2.41)\end{array}$ & $\begin{array}{l}1.20 \\
(0.65)\end{array}$ & $\begin{array}{l}2.94 \\
(0.31)\end{array}$ & $\begin{array}{l}0.02 \\
(0.02)\end{array}$ & $\begin{array}{l}0.81 \\
(0.12)\end{array}$ & $\begin{array}{l}0.72 \\
(0.08)\end{array}$ & $\begin{array}{l}0.24 \\
(0.04)\end{array}$ & $\begin{array}{l}2.39 \\
(0.32)\end{array}$ & $\begin{array}{l}0.22 \\
(0.76)\end{array}$ \\
\hline & E_1 & 13 & $\begin{array}{l}60.20 \\
(4.46)\end{array}$ & $\begin{array}{l}31.58 \\
(3.65)\end{array}$ & $\begin{array}{l}0.80 \\
(0.21)\end{array}$ & $\begin{array}{l}2.31 \\
(0.30)\end{array}$ & $\begin{array}{l}0.02 \\
(0.02)\end{array}$ & $\begin{array}{l}0.81 \\
(0.20)\end{array}$ & $\begin{array}{l}0.60 \\
(0.10)\end{array}$ & $\begin{array}{l}0.27 \\
(0.05)\end{array}$ & $\begin{array}{l}3.38 \\
(0.65)\end{array}$ & $\begin{array}{l}0.04 \\
(0.02)\end{array}$ \\
\hline & E_2 & 54 & $\begin{array}{l}55.95 \\
(2.23)\end{array}$ & $\begin{array}{l}34.35 \\
(1.95)\end{array}$ & $\begin{array}{l}0.99 \\
(0.80)\end{array}$ & $\begin{array}{l}2.32 \\
(0.23)\end{array}$ & $\begin{array}{l}0.02 \\
(0.02)\end{array}$ & $\begin{array}{l}0.94 \\
(0.19)\end{array}$ & $\begin{array}{l}0.89 \\
(0.17)\end{array}$ & $\begin{array}{l}0.55 \\
(0.12)\end{array}$ & $\begin{array}{l}3.87 \\
(0.78)\end{array}$ & $\begin{array}{l}0.12 \\
(0.04)\end{array}$ \\
\hline & F_2.589 & 26 & $\begin{array}{l}58.05 \\
(3.29)\end{array}$ & $\begin{array}{l}33.00 \\
(2.32)\end{array}$ & $\begin{array}{l}0.88 \\
(0.49)\end{array}$ & $\begin{array}{l}2.23 \\
(0.39)\end{array}$ & $\begin{array}{l}0.01 \\
(0.02)\end{array}$ & $\begin{array}{l}0.91 \\
(0.43)\end{array}$ & $\begin{array}{l}0.66 \\
(0.21)\end{array}$ & $\begin{array}{l}0.24 \\
(0.07)\end{array}$ & $\begin{array}{l}3.93 \\
(1.71)\end{array}$ & $\begin{array}{l}0.08 \\
(0.04)\end{array}$ \\
\hline & F_3.20 & 21 & $\begin{array}{l}59.93 \\
(3.09)\end{array}$ & $\begin{array}{l}32.30 \\
(2.70)\end{array}$ & $\begin{array}{l}0.83 \\
(0.62)\end{array}$ & $\begin{array}{l}2.40 \\
(0.42)\end{array}$ & $\begin{array}{l}0.01 \\
0.02\end{array}$ & $\begin{array}{l}0.81 \\
0.29\end{array}$ & $\begin{array}{l}0.58 \\
0.16\end{array}$ & $\begin{array}{l}0.33 \\
0.08\end{array}$ & $\begin{array}{l}2.73 \\
0.82\end{array}$ & $\begin{array}{l}0.07 \\
(0.04)\end{array}$ \\
\hline \multirow[t]{3}{*}{ Group 3} & CS_2016.41.2 & 31 & $\begin{array}{l}69.19 \\
(3.42)\end{array}$ & $\begin{array}{l}19.77 \\
(2.58)\end{array}$ & $\begin{array}{l}0.74 \\
(1.30)\end{array}$ & $\begin{array}{l}3.13 \\
(0.81)\end{array}$ & $\begin{array}{l}0.17 \\
(0.24)\end{array}$ & $\begin{array}{l}1.39 \\
(0.38)\end{array}$ & $\begin{array}{l}0.53 \\
(0.38)\end{array}$ & $\begin{array}{l}0.91 \\
(0.98)\end{array}$ & $\begin{array}{l}4.06 \\
(0.58)\end{array}$ & $\begin{array}{l}0.10 \\
(0.09)\end{array}$ \\
\hline & CS_1 & 29 & $\begin{array}{l}64.96 \\
(3.68)\end{array}$ & $\begin{array}{l}21.74 \\
(2.43)\end{array}$ & $\begin{array}{l}0.38 \\
(0.23)\end{array}$ & $\begin{array}{l}4.02 \\
(0.97)\end{array}$ & $\begin{array}{l}0.08 \\
(0.05)\end{array}$ & $\begin{array}{l}1.61 \\
(0.56)\end{array}$ & $\begin{array}{l}0.70 \\
(0.30)\end{array}$ & $\begin{array}{l}1.20 \\
(0.80)\end{array}$ & $\begin{array}{l}5.22 \\
(1.26)\end{array}$ & $\begin{array}{l}0.10 \\
(0.09)\end{array}$ \\
\hline & CS_3 & 17 & $\begin{array}{l}64.40 \\
(3.84)\end{array}$ & $\begin{array}{l}23.58 \\
(3.17)\end{array}$ & $\begin{array}{l}0.55 \\
(0.41)\end{array}$ & $\begin{array}{l}3.97 \\
(1.67)\end{array}$ & $\begin{array}{l}0.09 \\
(0.09)\end{array}$ & $\begin{array}{l}1.62 \\
(0.37)\end{array}$ & $\begin{array}{l}0.62 \\
(0.17)\end{array}$ & $\begin{array}{l}0.71 \\
(0.16)\end{array}$ & $\begin{array}{l}4.33 \\
(1.63)\end{array}$ & $\begin{array}{l}0.13 \\
(0.04)\end{array}$ \\
\hline Outlier & $\begin{array}{l}\text { E_Green glazed } \\
\text { grape tendrils } 7\end{array}$ & 42 & $\begin{array}{l}70.64 \\
(2.66)\end{array}$ & $\begin{array}{l}21.51 \\
(1.96)\end{array}$ & $\begin{array}{l}0.58 \\
(0.24)\end{array}$ & $\begin{array}{l}2.57 \\
(0.42)\end{array}$ & $\begin{array}{l}0.02 \\
(0.02)\end{array}$ & $\begin{array}{l}1.00 \\
(0.15)\end{array}$ & $\begin{array}{l}0.90 \\
(0.36)\end{array}$ & $\begin{array}{l}0.34 \\
(0.27)\end{array}$ & $\begin{array}{l}2.30 \\
(1.01)\end{array}$ & $\begin{array}{l}0.14 \\
(0.31)\end{array}$ \\
\hline
\end{tabular}

The italicized values in parenthesis represent the standard deviation, i.e. within \pm 1 standard deviations from the average

Specifically, the matrix of the ceramic bodies from Besztercebánya/Banská Bystrica and Csábrág/Čabrad' contain either less $\mathrm{Al}_{2} \mathrm{O}_{3}$ or more $\mathrm{K}_{2} \mathrm{O}$ than those from Eger, Szécsény, Salgó, and Fülek/Fil'akovo, respectively. Furthermore, one sample from Csábrág/Čabrad' (CS_1) has a higher average $\mathrm{K}_{2} \mathrm{O}$ content than the other samples.

The $\mathrm{SiO}_{2}-\mathrm{Al}_{2} \mathrm{O}_{3}-\mathrm{Na}_{2} \mathrm{O}+\mathrm{K}_{2} \mathrm{O}$ and $\mathrm{SiO}_{2}-\mathrm{Al}_{2} \mathrm{O}_{3}-\mathrm{CaO}$ ternary plots show that the raw material of the studied ceramics is non-calcareous clay (Fig. 6).

\section{Glaze}

Glazes with different colours (light green, dark green, yellow, orange, white, blue, and brown) were analysed in order to determine their production technology and colourants. Based on their microstructure and chemical composition (Table 2), the glazes of the three petrographic groups can be characterised as follows.

\section{Chemical composition}

The glazes of the analysed tiles are of very high-lead ( $>$ $60 \mathrm{wt} \% \mathrm{PbO}$ ), high-lead (40-60 wt\% PbO), and tinopacified lead type (according to Matin et al. 2018, Salinas et al. 2019b).

Tiles from Besztercebánya/Banská Bystrica, Salgó, Szécsény, Eger, and Fülek/Filakovo (typical BSE images of the glazes are in Fig. 7) have high-lead and very high-leadtype glazes (48-72 wt\% $\mathrm{PbO}$ ) (Table 2). The average of the total alkali content $\left(\mathrm{Na}_{2} \mathrm{O}+\mathrm{K}_{2} \mathrm{O}\right)$ is between 0.4 and $2.1 \mathrm{wt} \%$, except the glaze of the 'BB Adam and Eve' tile from Besztercebánya/Banská Bystrica, which exhibits a higher $\mathrm{K}_{2} \mathrm{O}$ content (5.7 wt\%) (Table 2, Fig. 8). Glazes of the tiles from Eger have the lowest total alkali content. Most of the tiles have green glazes with an average of $1-6 \mathrm{wt} \% \mathrm{CuO}$ content. In the yellow glazes $2-2.3 \mathrm{wt} \% \mathrm{FeO}$ was detected. Glazes on Besztercebánya/Banská Bystrica tiles (group 1) contain 0.3- 
Fig. $5 \mathrm{~K} 2 \mathrm{O} / \mathrm{SiO}_{2}-\mathrm{Al}_{2} \mathrm{O}_{3} / \mathrm{SiO}_{2}$ discrimination diagram showing the matrix composition of the ceramic body of tiles measured by EPMA-WDS (according to the data shown in Table 1; error bars indicate standard deviation; $\mathrm{BB}$, Besztercebánya/Banská Bystrica; CS, Csábrág/Čabrad'; E, Eger; F, Fülek/Fil'akovo; S, Salgó; SZ, Szécsény; $\mathrm{m}$, mullite detected by XRD)

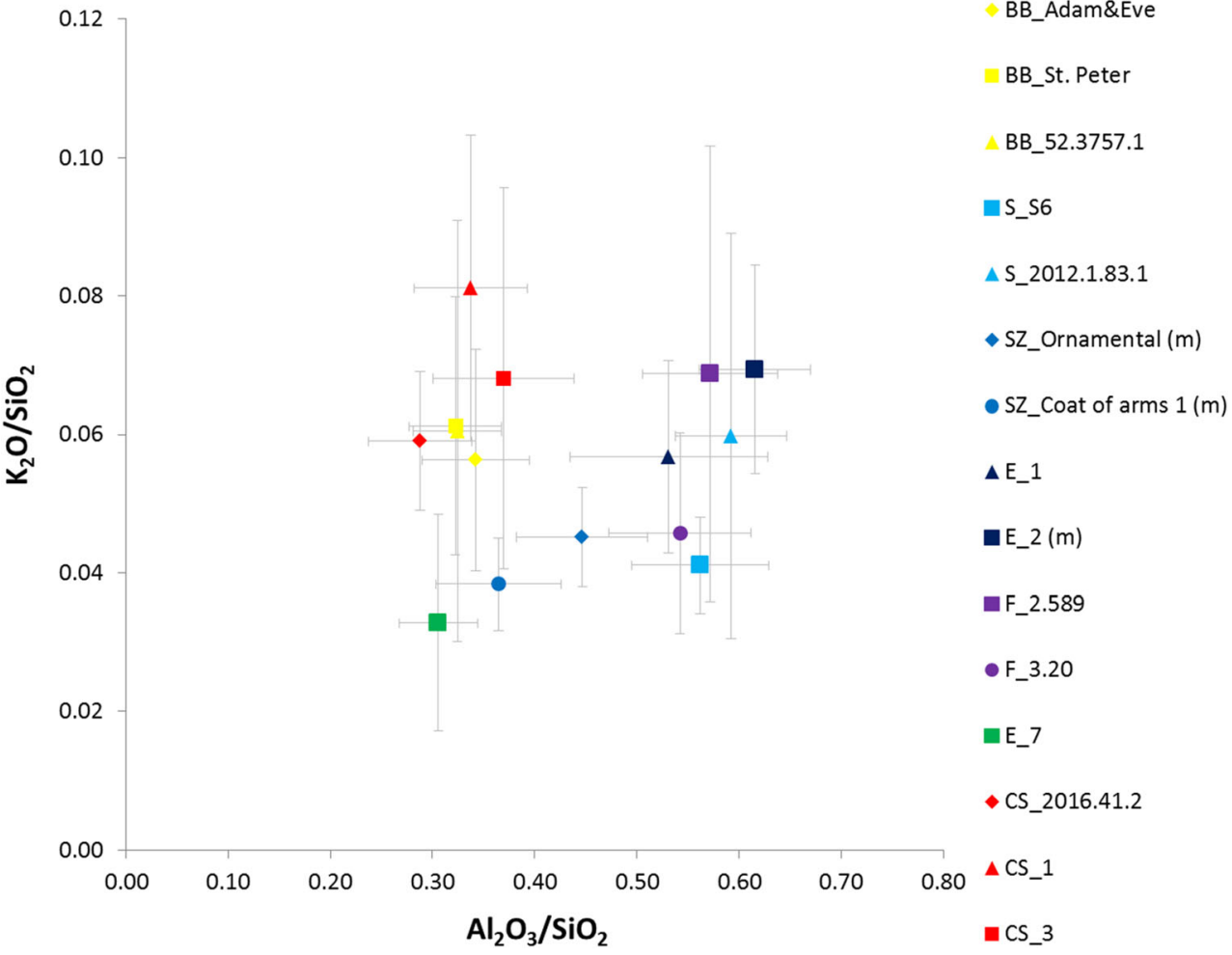

$1.4 \mathrm{wt} \% \mathrm{Sb}_{2} \mathrm{O}_{3}$ in the vitreous matrix of all tiles. Some tiles from Northern Hungary (Salgó, Szécsény, Eger) and Fülek/ Filakovo (group 2) are the exceptions: (i) The green glaze of one polychrome tile from Eger ('E_2', yellow and green glaze) contained dissolved tin $\left(5.3 \pm 3.0 \mathrm{wt} \% \mathrm{SnO}_{2}\right)$ and iron $(2.1 \pm 1 \mathrm{wt} \% \mathrm{FeO})$ in the vitreous matrix, without any discrete tin-bearing particles. (ii) One polychrome tile from Fülek/ Filakovo ('F_2.589' with green, brown, and light brownishwhite glazes) showed a significant amount of tin in the brown and light brownish-white glazes $\left(2-11 \mathrm{wt} \% \mathrm{SnO}_{2}\right.$, according to area EDS measurements). The highest tin content, in addition to some sodium, was measured in the light brownishwhite glaze. In the brown glaze, 5-7.3 wt\% $\mathrm{CuO}$ and 2.2$4 \mathrm{wt} \% \mathrm{FeO}$ were detected.

Fig. $6 \mathrm{SiO}_{2}-\mathrm{Al}_{2} \mathrm{O}_{3}-\mathrm{Na}_{2} \mathrm{O}+\mathrm{K}_{2} \mathrm{O}$ and $\mathrm{SiO}_{2}-\mathrm{Al}_{2} \mathrm{O}_{3}-\mathrm{CaO}$ ternary plots showing the matrix composition of the ceramic body of tiles measured by EPMA-WDS (according to the data shown in Table 1, after Schairer and Bowen 1947 and Heimann 2010)

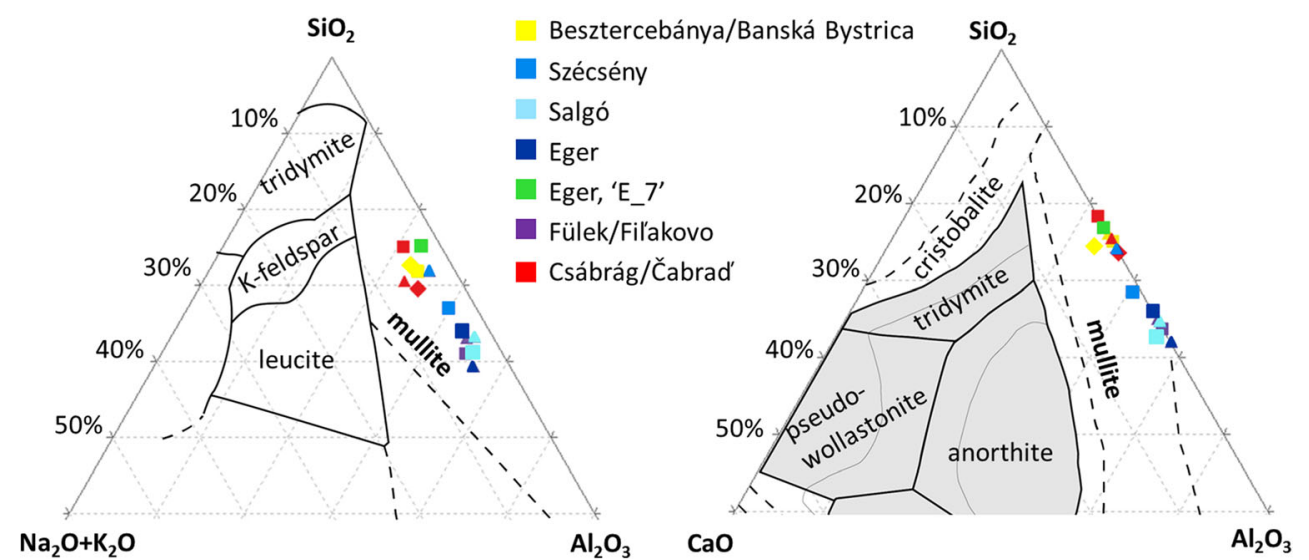

Tiles from Csábrág/Čabrad' are diverse both in colour and composition (group 3). Four tiles had tin-opacified high- and very high-lead glazes with 45 to $71 \mathrm{wt} \% \mathrm{PbO}$ and an average of $1.7-18 \mathrm{wt} \% \mathrm{SnO}_{2}$ content according to area EDS measurements (Fig. 9a-d, Table 2). The wide $\mathrm{SnO}_{2}$ concentration range is due to the significant difference between the different colours of the same tiles as white and blue glazes contain more tin than green glazes do (Table 2). The total alkali $\left(\mathrm{Na}_{2} \mathrm{O}+\right.$ $\mathrm{K}_{2} \mathrm{O}$ ) content varies between 0.4 and $4 \mathrm{wt} \%$. The total alkali content for yellow colours is often very low $(0.4-0.6 \mathrm{wt} \%)$, whereas the $\mathrm{Na}_{2} \mathrm{O}$ content in the white and blue glazes (e.g. 'CS_2016.89.3', 'CS_2016.117.3', and 'CS 2016.163.12') is higher (1.8 to $4 \mathrm{wt} \%)$ (Table 2, Fig. 8). In the green glazes, $0.6-2.5 \mathrm{wt} \% \mathrm{CuO}$, while in the blue glaze, $0.36 \mathrm{wt} \% \mathrm{CoO}$ is 
Table 2 Chemical composition of the glazes of each petrographic group (in wt\%, average of area and spot measurements performed by EDS, standard deviation in parenthesis, * - average of area measurements, $* *$ - average of spot measurements only, $\mathrm{c}-$ colour, $\mathrm{n}-$ number of measurements, Bl - blue glaze, $\mathrm{Br}$ - brown glaze, BW - light brownish-white glaze, DG - dark green glaze, $\mathrm{G}$ - green glaze, $\mathrm{W}$ - white glaze, Y - yellow glaze, BB - Besztercebánya/Banská Bystrica, CS - Csábrág/Čabrad', E - Eger, F - Fülek/Fil'akovo, S - Salgó, SZ - Szécsény)

\begin{tabular}{|c|c|c|c|c|c|c|c|c|c|c|c|c|c|c|c|c|c|c|}
\hline & Sample & $c$ & $n$ & $\mathrm{SiO}_{2}$ & $\mathrm{PbO}$ & $\mathrm{SnO}_{2}$ & $\mathrm{Na}_{2} \mathrm{O}$ & $\mathrm{K}_{2} \mathrm{O}$ & $\mathrm{CaO}$ & $\mathrm{MgO}$ & $\mathrm{Al}_{2} \mathrm{O}_{3}$ & $\mathrm{FeO}$ & $\mathrm{TiO}_{2}$ & $\mathrm{CuO}$ & $\mathrm{Sb}_{2} \mathrm{O}_{3}$ & $\mathrm{CoO}$ & $\mathrm{MnO}$ & Total \\
\hline \multirow[t]{27}{*}{$\begin{array}{c}\text { Group } \\
1\end{array}$} & $\begin{array}{l}\text { BB_Adam and } \\
\text { Eve }\end{array}$ & G & 17 & $\begin{array}{l}23.17 \\
(0.61)\end{array}$ & $\begin{array}{l}62.90 \\
(1.29)\end{array}$ & - & $\begin{array}{l}0.02 \\
(0.11)\end{array}$ & $\begin{array}{l}5.69 \\
(1.58)\end{array}$ & $\begin{array}{l}0.63 \\
(0.19)\end{array}$ & $\begin{array}{l}0.42 \\
(0.30)\end{array}$ & $\begin{array}{l}3.77 \\
(0.32)\end{array}$ & $\begin{array}{l}0.64 \\
(0.09)\end{array}$ & $\begin{array}{l}0.12 \\
(0.11)\end{array}$ & $\begin{array}{l}2.20 \\
(0.39)\end{array}$ & 1.43 & - & - & $\begin{array}{l}99.65 \\
(0.86)\end{array}$ \\
\hline & \multirow[t]{2}{*}{ BB_St. Peter } & $\mathrm{G}$ & 18 & 26.42 & 61.30 & - & 0.05 & 0.58 & 0.50 & 0.62 & 5.59 & 0.88 & 0.26 & 2.28 & 0.83 & - & - & 99.30 \\
\hline & & & & $(0.51)$ & $(0.66)$ & & $(0.09)$ & $(0.12)$ & $(0.05)$ & $(0.46)$ & $(0.30)$ & $(0.10)$ & $(0.23)$ & $(0.37)$ & $(0.26)$ & & & $(0.94)$ \\
\hline & BB_314 & G & 9 & 25.71 & 65.79 & - & 0.20 & 0.47 & 0.46 & 0.36 & 4.00 & 0.80 & 0.12 & 1.42 & 0.67 & - & - & 99.69 \\
\hline & & & & $(1.75)$ & (1.67) & & $(0.08)$ & (0.13) & $(0.11)$ & $(0.04)$ & $(0.54)$ & $(0.08)$ & $(0.05)$ & $(0.65)$ & $(0.15)$ & & & (1.04) \\
\hline & BB_316 & G & 15 & 32.69 & 53.30 & - & 0.29 & 0.99 & 0.65 & 0.52 & 5.25 & 1.14 & 0.24 & 2.87 & 1.39 & - & - & 99.33 \\
\hline & & & & $(1.30)$ & (1.13) & & $(0.05)$ & $(0.10)$ & $(0.08)$ & $(0.06)$ & $(0.60)$ & $(0.14)$ & $(0.05)$ & $(0.77)$ & (0.16) & & & $(0.60)$ \\
\hline & BB_3589a & G & 15 & 33.35 & 51.55 & - & 0.23 & 1.00 & 1.20 & 0.62 & 5.43 & 1.21 & 0.24 & 3.93 & 1.46 & - & - & 99.82 \\
\hline & & & & (1.79) & $(1.10)$ & & $(0.06)$ & $(0.12)$ & $(0.32)$ & $(0.09)$ & $(0.44)$ & $(0.12)$ & $(0.05)$ & $(1.81)$ & $(0.26)$ & & & $(1.14)$ \\
\hline & BB_3591 & G & 11 & 25.81 & 65.05 & - & 0.26 & 0.65 & 0.28 & 0.38 & 4.01 & 0.76 & 0.14 & 2.30 & 0.33 & - & - & 99.69 \\
\hline & & & & $(0.98)$ & (1.08) & & $(0.05)$ & $(0.11)$ & $(0.04)$ & $(0.03)$ & $(0.47)$ & $(0.06)$ & $(0.05)$ & $(0.65)$ & $(0.03$ & & & $(0.90)$ \\
\hline & BB_6544 & G & 22 & 30.11 & 56.93 & - & 1.43 & 0.73 & 0.77 & 0.39 & 4.28 & 0.81 & 0.24 & 3.17 & 0.99 & - & 0.90 & 98.51 \\
\hline & & & & $(2.58)$ & (1.59) & & $(1.78)$ & $(0.16)$ & $(0.10)$ & $(0.05)$ & $(0.76)$ & $(0.18)$ & $(0.13)$ & (1.12) & $(0.36)$ & & (1.51) & $(1.73)$ \\
\hline & BB_6545 & G & 13 & 30.97 & 57.54 & - & 0.18 & 0.67 & 1.07 & 0.46 & 3.98 & 1.15 & 0.18 & 2.22 & 1.14 & - & - & 99.56 \\
\hline & & & & $(1.76)$ & (1.44) & & $(0.06)$ & (0.07) & $(0.42)$ & (0.06) & $(0.41)$ & $(0.39)$ & $(0.06)$ & $(0.71)$ & (0.39) & & & $(0.66)$ \\
\hline & BB_6547 & G & 9 & 35.65 & 53.09 & - & 0.11 & 0.65 & 0.84 & 0.37 & 4.25 & 0.85 & 0.24 & 2.67 & 0.96 & - & - & 99.68 \\
\hline & & & & $(2.25)$ & $(2.48)$ & & $(0.07)$ & $(0.20)$ & $(0.54)$ & (0.04) & $(0.69)$ & $(0.11)$ & $(0.07)$ & (1.07) & (0.16) & & & $(0.83)$ \\
\hline & BB_6549 & G & 10 & 24.88 & 63.95 & - & 0.20 & 0.32 & 0.59 & 0.58 & 5.39 & 1.14 & 0.29 & 2.40 & - & - & - & 99.73 \\
\hline & & & & $(0.73)$ & $(2.23)$ & & $(0.13)$ & $(0.11)$ & $(0.12)$ & $(0.08)$ & $(0.17)$ & $(0.14)$ & $(0.08)$ & $(1.73)$ & & & & $(0.59)$ \\
\hline & BB_6554 & G & 11 & 23.32 & 67.15 & - & 0.19 & 0.52 & 0.60 & 0.40 & 4.28 & 0.79 & 0.15 & 1.55 & 1.14 & - & - & 100.10 \\
\hline & & & & (1.17) & $(0.82)$ & & $(0.06)$ & $(0.08)$ & $(0.05)$ & $(0.06)$ & $(0.32)$ & $(0.10)$ & $(0.07)$ & $(0.37)$ & $(0.38)$ & & & $(0.84)$ \\
\hline & BB_6555 & G & 15 & 27.15 & 63.64 & - & 0.14 & 0.45 & 0.38 & 0.43 & 3.97 & 1.23 & 0.18 & 2.25 & - & - & - & 99.83 \\
\hline & & & & (1.12) & $(1.53)$ & & $(0.05)$ & $(0.05)$ & $(0.05)$ & (0.04) & $(0.36)$ & $(0.46)$ & $(0.06)$ & $(0.35)$ & & & & $(0.58)$ \\
\hline & BB_6557 & G & 14 & 26.02 & 64.59 & - & 0.07 & 0.31 & 0.62 & 0.29 & 2.81 & 0.54 & 0.09 & 3.50 & 0.95 & - & - & 99.79 \\
\hline & & & & (1.01) & (1.09) & & $(0.05)$ & $(0.05)$ & $(0.08)$ & $(0.03)$ & $(0.34)$ & $(0.07)$ & $(0.07)$ & $(0.66)$ & $(0.24)$ & & & $(0.69)$ \\
\hline & BB_95.242.1 & G & 11 & 27.73 & 62.72 & - & 0.15 & 0.69 & 0.72 & 0.32 & 3.70 & 0.71 & 0.14 & 2.18 & 1.01 & - & - & 100.08 \\
\hline & & & & (1.12) & $(1.25)$ & & $(0.06)$ & $(0.38)$ & $(0.21)$ & (0.04) & $(0.47)$ & $(0.07)$ & $(0.06)$ & $(0.94)$ & $(0.18)$ & & & $(0.81)$ \\
\hline Group & SZ_Ornamental & G & 24 & 29.01 & 54.50 & - & 0.26 & 0.73 & 2.22 & 0.32 & 9.62 & 0.82 & 0.54 & 1.00 & - & - & - & 99.00 \\
\hline 2 & & & & $(3.06)$ & $(5.35)$ & & $(0.18)$ & $(0.49)$ & $(0.22)$ & $(0.05)$ & $(2.45)$ & $(0.14)$ & $(0.10)$ & $(0.28)$ & & & & $(0.65)$ \\
\hline & SZ_Coat of arms & G & 31 & 21.04 & 64.98 & - & 0.11 & 0.54 & 0.36 & 1.04 & 6.81 & 0.70 & 1.86 & 2.32 & - & - & - & 99.74 \\
\hline & 1 & & & (1.63) & $(1.83)$ & & $(0.08)$ & $(0.13)$ & $(0.16)$ & $(0.51)$ & $(0.40)$ & $(0.12)$ & $(0.34)$ & $(0.71)$ & & & & $(0.72)$ \\
\hline & SZ_Coat of arms & G & 21 & 19.47 & 70.56 & - & 0.10 & 0.42 & 0.20 & 0.20 & 5.46 & 0.50 & 0.38 & 2.77 & - & - & - & 100.06 \\
\hline & $\overline{2}$ & & & $(1.44)$ & $(0.86)$ & & $(0.10)$ & (0.03) & $(0.04)$ & (0.03) & $(0.56)$ & $(0.06)$ & $(0.05)$ & $(0.58)$ & & & & $(0.98)$ \\
\hline & SZ_St. Peter & G & 21 & 30.17 & 67.11 & 0.82 & 0.16 & 0.78 & 3.08 & 0.60 & 7.50 & 0.86 & 0.54 & 1.78 & - & - & - & 98.85 \\
\hline & & & & $(0.84)$ & $(1.06)$ & $(0.06)$ & $(0.11)$ & $(0.15)$ & $(0.31)$ & $(0.06)$ & $(0.42)$ & $(0.08)$ & $(0.10)$ & $(0.36)$ & & & & $(0.66)$ \\
\hline & SZ_Figure of a & G & 19 & 23.42 & 63.72 & - & 0.15 & 0.44 & 0.36 & 0.24 & 6.95 & 0.58 & 0.45 & 2.45 & - & - & - & 98.76 \\
\hline & Saint & & & (1.34) & $(1.17)$ & & $(0.10)$ & $(0.07)$ & $(0.07)$ & $(0.05)$ & $(0.32)$ & $(0.08)$ & $(0.07)$ & $(0.71)$ & & & & $(0.62)$ \\
\hline & SZ_Cloak & G & 12 & 21.96 & 67.11 & - & 0.34 & 0.46 & 0.24 & 0.24 & 5.69 & 0.55 & 0.38 & 1.88 & - & - & - & 98.85 \\
\hline & & & & (2.02) & (1.06) & & $(0.31)$ & (0.09) & $(0.04)$ & $(0.03)$ & (1.12) & $(0.07)$ & $(0.05)$ & $(0.34)$ & & & & $(0.66)$ \\
\hline & SZ_Hand holding & G & 14 & 25.25 & 58.57 & 0.56 & 0.01 & 0.30 & 0.68 & 0.46 & 7.55 & 0.87 & 0.51 & 5.05 & 0.10 & - & - & 99.88 \\
\hline & Globus & & & (1.41) & $(2.72)$ & $(0.06)$ & $(0.17)$ & $(0.26)$ & (0.07) & $(0.06)$ & (1.58) & $(0.11)$ & $(0.12)$ & $(1.08)$ & $(0.06)$ & & & (1.04) \\
\hline & SZ_S7 & G & 22 & 31.68 & 52.66 & 0.85 & 0.23 & 1.24 & 1.14 & 0.48 & 7.56 & 1.03 & 0.52 & 2.17 & 0.11 & - & - & 99.65 \\
\hline & & & & (1.64) & $(2.41)$ & $(0.14)$ & $(0.07)$ & $(0.28)$ & $(0.37)$ & $(0.08)$ & $(0.57)$ & $(0.16)$ & $(0.10)$ & $(0.72)$ & $(0.06)$ & & & $(0.75)$ \\
\hline & SZ_S8 & G & 21 & 27.12 & 59.23 & - & 0.48 & 0.30 & 1.02 & 0.42 & 7.33 & 0.73 & 0.40 & 2.74 & - & - & - & 100.15 \\
\hline & & & & (1.27) & $(1.23)$ & & $(0.13)$ & $(0.13)$ & $(0.15)$ & $(0.07)$ & (0.39) & $(0.07)$ & $(0.06)$ & $(0.45)$ & & & & (1.09) \\
\hline & SZ_S9 & G & 11 & 22.13 & 66.03 & - & 0.17 & 0.44 & 0.24 & 0.24 & 6.92 & 0.64 & 0.43 & 2.24 & - & - & - & 99.47 \\
\hline & & & & $(0.88)$ & $(0.56)$ & & $(0.06)$ & $(0.02)$ & (0.04) & $(0.03)$ & $(0.25)$ & $(0.07)$ & $(0.07)$ & $(0.51)$ & & & & $(0.74)$ \\
\hline & S_2012.1.83.3 & G & 29 & 29.96 & 58.09 & - & 0.22 & 0.60 & 0.30 & 0.24 & 6.84 & 0.68 & 0.58 & 1.73 & - & - & - & 99.25 \\
\hline & & & & (1.06) & $(2.30)$ & & $(0.06)$ & $(0.20)$ & $(0.07)$ & $(0.05)$ & (1.10) & $(0.15)$ & $(0.55)$ & $(0.34)$ & & & & $(0.82)$ \\
\hline & S_2012.1.83.5 & G & 11 & 24.90 & 63.09 & - & 1.01 & 0.34 & 0.38 & 0.49 & 6.38 & 0.68 & 0.29 & 1.95 & - & - & - & 99.26 \\
\hline & & & & $(2.64)$ & $(2.72)$ & & $(0.71)$ & $(0.19)$ & $(0.22)$ & $(0.29)$ & $(0.90)$ & $(0.15)$ & $(0.07)$ & $(0.46)$ & & & & $(1.02)$ \\
\hline & S_2012.1.83.13 & G & 15 & 19.77 & 70.43 & - & 0.02 & 0.41 & 0.44 & 0.24 & 5.46 & 1.02 & 0.26 & 2.43 & - & - & - & 100.45 \\
\hline & & & & $(0.41)$ & $(0.86)$ & & $(0.03)$ & $(0.04)$ & $(0.06)$ & $(0.03)$ & $(0.53)$ & $(0.08)$ & $(0.04)$ & $(0.17)$ & & & & $(0.69)$ \\
\hline & S_2012.1.83.14 & G & 12 & 20.20 & 68.61 & - & 0.07 & 0.42 & 0.45 & 0.24 & 5.45 & 0.93 & 0.27 & 3.39 & - & - & - & 100.03 \\
\hline & & & & $(0.72)$ & $(0.72)$ & & $(0.24)$ & $(0.08)$ & $(0.07)$ & $(0.04)$ & $(0.57)$ & $(0.10)$ & $(0.05)$ & $(0.61)$ & & & & $(0.90)$ \\
\hline & S_2012.1.84.19 & G & 14 & 28.83 & 60.61 & - & 0.19 & 0.48 & 0.17 & 0.21 & 5.98 & 0.55 & 0.31 & 2.72 & - & - & - & 100.04 \\
\hline & & & & $(0.86)$ & $(1.71)$ & & $(0.11)$ & $(0.08)$ & $(0.03)$ & $(0.04)$ & $(0.88)$ & $(0.07)$ & $(0.05)$ & $(0.87)$ & & & & $(0.60)$ \\
\hline & S_2012.1.84.20 & G & 16 & 26.97 & 64.49 & - & 0.23 & 0.42 & 0.17 & 0.16 & 4.69 & 0.51 & 0.38 & 1.82 & - & - & - & 99.84 \\
\hline & & & & $(0.77)$ & $(1.32)$ & & $(0.07)$ & (0.09) & $(0.11)$ & $(0.05)$ & $(0.97)$ & $(0.13)$ & $(0.23)$ & $(0.41)$ & & & & $(0.54)$ \\
\hline & S_2012.1.84.21 & G & 16 & 27.83 & 61.24 & - & 0.07 & 0.57 & 0.17 & 0.22 & 6.56 & 0.60 & 0.34 & 1.82 & - & - & - & 99.41 \\
\hline & & & & $(2.06)$ & $(1.72)$ & & $(0.06)$ & $(0.15)$ & $(0.06)$ & $(0.05)$ & $(0.52)$ & $(0.07)$ & $(0.05)$ & $(0.70)$ & & & & $(0.95)$ \\
\hline
\end{tabular}


Table 2 (continued)

\begin{tabular}{|c|c|c|c|c|c|c|c|c|c|c|c|c|c|c|c|c|c|}
\hline Sample & $c$ & $n$ & $\mathrm{SiO}_{2}$ & $\mathrm{PbO}$ & $\mathrm{SnO}_{2}$ & $\mathrm{Na}_{2} \mathrm{O}$ & $\mathrm{K}_{2} \mathrm{O}$ & $\mathrm{CaO}$ & $\mathrm{MgO}$ & $\mathrm{Al}_{2} \mathrm{O}_{3}$ & $\mathrm{FeO}$ & $\mathrm{TiO}_{2}$ & $\mathrm{CuO}$ & $\mathrm{Sb}_{2} \mathrm{O}_{3}$ & $\mathrm{CoO}$ & $\mathrm{MnO}$ & Total \\
\hline S_2012.1.84.23 & G & 21 & $\begin{array}{l}22.53 \\
(1.25)\end{array}$ & $\begin{array}{l}71.14 \\
(1.73)\end{array}$ & - & $\begin{array}{l}0.16 \\
(0.08)\end{array}$ & $\begin{array}{l}0.30 \\
(0.09)\end{array}$ & $\begin{array}{l}0.24 \\
(0.07)\end{array}$ & $\begin{array}{l}0.13 \\
(0.05)\end{array}$ & $\begin{array}{l}3.29 \\
(0.90)\end{array}$ & $\begin{array}{l}0.32 \\
(0.07)\end{array}$ & $\begin{array}{l}0.18 \\
(0.07)\end{array}$ & $\begin{array}{l}1.48 \\
(0.52)\end{array}$ & - & - & - & $\begin{array}{l}99.76 \\
(0.79)\end{array}$ \\
\hline S_2012.1.84.28 & G & 20 & $\begin{array}{l}22.98 \\
(0.88)\end{array}$ & $\begin{array}{l}67.20 \\
(0.84)\end{array}$ & - & $\begin{array}{l}0.09 \\
(0.12)\end{array}$ & $\begin{array}{l}0.56 \\
(0.09)\end{array}$ & $\begin{array}{l}0.23 \\
(0.04)\end{array}$ & $\begin{array}{l}0.21 \\
(0.04)\end{array}$ & $\begin{array}{l}5.62 \\
(0.41)\end{array}$ & $\begin{array}{l}1.34 \\
(0.08)\end{array}$ & $\begin{array}{l}0.26 \\
(0.05)\end{array}$ & $\begin{array}{l}1.70 \\
(0.46)\end{array}$ & - & - & - & $\begin{array}{l}100.18 \\
(0.86)\end{array}$ \\
\hline S_2012.1.85.2 & G & 9 & $\begin{array}{l}30.10 \\
(1.37)\end{array}$ & $\begin{array}{l}52.79 \\
(0.62)\end{array}$ & - & $\begin{array}{l}0.14 \\
(0.04)\end{array}$ & $\begin{array}{l}0.64 \\
(0.07)\end{array}$ & $\begin{array}{l}1.09 \\
(0.10)\end{array}$ & $\begin{array}{l}0.35 \\
(0.05)\end{array}$ & $\begin{array}{l}8.75 \\
(0.44)\end{array}$ & $\begin{array}{l}0.87 \\
(0.11)\end{array}$ & $\begin{array}{l}0.38 \\
(0.09)\end{array}$ & $\begin{array}{l}4.89 \\
(0.32)\end{array}$ & - & - & - & $\begin{array}{l}100.01 \\
(0.62)\end{array}$ \\
\hline S_2012.1.86.1 & G & 14 & $\begin{array}{l}23.28 \\
(1.01)\end{array}$ & $\begin{array}{l}64.34 \\
(0.80)\end{array}$ & - & $\begin{array}{l}0.02 \\
(0.06)\end{array}$ & $\begin{array}{l}0.43 \\
(0.07)\end{array}$ & $\begin{array}{l}0.64 \\
(0.06)\end{array}$ & $\begin{array}{l}0.30 \\
(0.05)\end{array}$ & $\begin{array}{l}7.21 \\
(0.64)\end{array}$ & $\begin{array}{l}1.06 \\
(0.07)\end{array}$ & $\begin{array}{l}0.40 \\
(0.21)\end{array}$ & $\begin{array}{l}1.67 \\
(0.20)\end{array}$ & - & - & - & $\begin{array}{l}99.36 \\
(0.94)\end{array}$ \\
\hline S_2012.1.86.3 & G & 10 & $\begin{array}{l}31.51 \\
(1.91)\end{array}$ & $\begin{array}{l}54.34 \\
(1.87)\end{array}$ & - & $\begin{array}{l}0.14 \\
(0.04)\end{array}$ & $\begin{array}{l}0.94 \\
(0.22)\end{array}$ & $\begin{array}{l}0.55 \\
(0.08)\end{array}$ & $\begin{array}{l}0.27 \\
(0.04)\end{array}$ & $\begin{array}{l}9.27 \\
(0.50)\end{array}$ & $\begin{array}{l}0.91 \\
(0.13)\end{array}$ & $\begin{array}{l}0.40 \\
(0.05)\end{array}$ & $\begin{array}{l}1.80 \\
(0.38)\end{array}$ & - & - & - & $\begin{array}{l}100.13 \\
(0.59)\end{array}$ \\
\hline S_2012.1.87.1 & $\mathrm{G}$ & 14 & $\begin{array}{l}20.73 \\
(1.77)\end{array}$ & $\begin{array}{l}70.04 \\
(2.08)\end{array}$ & - & $\begin{array}{l}0.08 \\
(0.06)\end{array}$ & $\begin{array}{l}0.49 \\
(0.07)\end{array}$ & $\begin{array}{l}0.63 \\
(0.10)\end{array}$ & $\begin{array}{l}0.21 \\
(0.04)\end{array}$ & $\begin{array}{l}4.57 \\
(0.39)\end{array}$ & $\begin{array}{l}1.35 \\
(0.16)\end{array}$ & $\begin{array}{l}0.18 \\
(0.04)\end{array}$ & $\begin{array}{l}1.30 \\
(0.27)\end{array}$ & - & - & - & $\begin{array}{l}99.58 \\
(0.71)\end{array}$ \\
\hline S_2012.1.88.4 & $\mathrm{G}$ & 15 & $\begin{array}{l}19.64 \\
(0.42)\end{array}$ & $\begin{array}{l}71.62 \\
(1.42)\end{array}$ & - & $\begin{array}{l}0.09 \\
(0.06)\end{array}$ & $\begin{array}{l}0.32 \\
(0.05)\end{array}$ & $\begin{array}{l}0.50 \\
(0.10)\end{array}$ & $\begin{array}{l}0.19 \\
(0.06)\end{array}$ & $\begin{array}{l}4.53 \\
(1.05)\end{array}$ & $\begin{array}{l}0.81 \\
(0.14)\end{array}$ & $\begin{array}{l}0.23 \\
(0.08)\end{array}$ & $\begin{array}{l}2.06 \\
(0.24)\end{array}$ & - & - & - & $\begin{array}{l}99.98 \\
(0.56)\end{array}$ \\
\hline S_S4 & G & 12 & $\begin{array}{l}20.17 \\
(0.38)\end{array}$ & $\begin{array}{l}69.58 \\
(0.72)\end{array}$ & - & $\begin{array}{l}0.11 \\
(0.04)\end{array}$ & $\begin{array}{l}0.44 \\
(0.05)\end{array}$ & $\begin{array}{l}0.31 \\
(0.05)\end{array}$ & $\begin{array}{l}0.23 \\
(0.05)\end{array}$ & $\begin{array}{l}5.65 \\
(0.22)\end{array}$ & $\begin{array}{l}1.07 \\
(0.05)\end{array}$ & $\begin{array}{l}0.25 \\
(0.04)\end{array}$ & $\begin{array}{l}2.01 \\
(0.19)\end{array}$ & - & - & - & $\begin{array}{l}99.81 \\
(0.79)\end{array}$ \\
\hline S_S5 & G & 23 & $\begin{array}{l}30.81 \\
(2.34)\end{array}$ & $\begin{array}{l}61.32 \\
(3.57)\end{array}$ & - & $\begin{array}{l}0.87 \\
(0.60)\end{array}$ & $\begin{array}{l}0.53 \\
(0.23)\end{array}$ & $\begin{array}{l}0.35 \\
(0.06)\end{array}$ & $\begin{array}{l}0.15 \\
(0.06)\end{array}$ & $\begin{array}{l}3.61 \\
(1.18)\end{array}$ & $\begin{array}{l}0.34 \\
(0.11)\end{array}$ & $\begin{array}{l}0.26 \\
(0.08)\end{array}$ & $\begin{array}{l}1.16 \\
(0.27)\end{array}$ & - & - & - & $\begin{array}{l}99.40 \\
(0.92)\end{array}$ \\
\hline E_1 & G & 10 & $\begin{array}{l}27.06 \\
(1.13)\end{array}$ & $\begin{array}{l}62.39 \\
(1.62)\end{array}$ & - & $\begin{array}{l}0.40 \\
(0.11)\end{array}$ & $\begin{array}{l}0.38 \\
(0.12)\end{array}$ & $\begin{array}{l}0.48 \\
(0.33)\end{array}$ & $\begin{array}{l}0.23 \\
(0.07)\end{array}$ & $\begin{array}{l}5.21 \\
(0.58)\end{array}$ & $\begin{array}{l}0.68 \\
(0.04)\end{array}$ & $\begin{array}{l}0.28 \\
(0.04)\end{array}$ & $\begin{array}{l}3.18 \\
(0.60)\end{array}$ & - & - & - & $\begin{array}{l}100.29 \\
(1.15)\end{array}$ \\
\hline & $\mathrm{Y}$ & 14 & $\begin{array}{l}26.68 \\
(1.07)\end{array}$ & $\begin{array}{l}66.18 \\
(2.44)\end{array}$ & - & $\begin{array}{l}0.56 \\
(0.33)\end{array}$ & $\begin{array}{l}0.40 \\
(0.10)\end{array}$ & $\begin{array}{l}0.17 \\
(0.21)\end{array}$ & $\begin{array}{l}0.19 \\
(0.06)\end{array}$ & $\begin{array}{l}4.81 \\
(1.01)\end{array}$ & $\begin{array}{l}0.52 \\
(0.11)\end{array}$ & $\begin{array}{l}0.15 \\
(0.08)\end{array}$ & - & $\begin{array}{l}2.23 \\
(2.67)\end{array}$ & - & - & $\begin{array}{l}100.29 \\
(0.69)\end{array}$ \\
\hline E_2 & G & 11 & $\begin{array}{l}23.43 \\
(0.76)\end{array}$ & $\begin{array}{l}64.14 \\
(3.99)\end{array}$ & $\begin{array}{l}5.26 \\
(3.01)\end{array}$ & $\begin{array}{l}0.00 \\
(0.15)\end{array}$ & $\begin{array}{l}0.30 \\
(0.15)\end{array}$ & $\begin{array}{l}0.58 \\
(0.25)\end{array}$ & $\begin{array}{l}0.26 \\
(0.07)\end{array}$ & $\begin{array}{l}3.40 \\
(1.04)\end{array}$ & $\begin{array}{l}2.08 \\
(0.95)\end{array}$ & $\begin{array}{l}0.15 \\
(0.06)\end{array}$ & $\begin{array}{l}5.64 \\
(2.84)\end{array}$ & - & - & - & $\begin{array}{l}99.25 \\
(0.50)\end{array}$ \\
\hline & $\mathrm{Y}$ & 4 & $\begin{array}{l}23.06 \\
(0.35)\end{array}$ & $\begin{array}{l}69.91 \\
(0.62)\end{array}$ & $\begin{array}{l}0.41 \\
(0.06)\end{array}$ & $\begin{array}{l}0.00 \\
(0.05)\end{array}$ & $\begin{array}{l}0.19 \\
(0.16)\end{array}$ & $\begin{array}{l}0.42 \\
(0.26)\end{array}$ & $\begin{array}{l}0.21 \\
(0.02)\end{array}$ & $\begin{array}{l}3.21 \\
(0.23)\end{array}$ & $\begin{array}{l}2.35 \\
(0.21)\end{array}$ & $\begin{array}{l}0.14 \\
(0.04)\end{array}$ & $\begin{array}{l}0.65 \\
(0.68)\end{array}$ & - & - & - & $\begin{array}{l}100.18 \\
(0.33)\end{array}$ \\
\hline E_3 & G & 23 & $\begin{array}{l}21.67 \\
(1.69)\end{array}$ & $\begin{array}{l}68.56 \\
(1.28)\end{array}$ & - & $\begin{array}{l}0.05 \\
(0.17)\end{array}$ & $\begin{array}{l}0.29 \\
(0.05)\end{array}$ & $\begin{array}{l}0.18 \\
(0.05)\end{array}$ & $\begin{array}{l}0.22 \\
(0.05)\end{array}$ & $\begin{array}{l}5.20 \\
(1.24)\end{array}$ & $\begin{array}{l}0.66 \\
(0.13)\end{array}$ & $\begin{array}{l}0.31 \\
(0.10)\end{array}$ & $\begin{array}{l}2.53 \\
(0.52)\end{array}$ & - & - & - & $\begin{array}{l}99.67 \\
(0.94)\end{array}$ \\
\hline E_ 4 & G & 21 & $\begin{array}{l}29.02 \\
(1.00)\end{array}$ & $\begin{array}{l}62.13 \\
(2.11)\end{array}$ & - & $\begin{array}{l}0.02 \\
(0.05)\end{array}$ & $\begin{array}{l}0.38 \\
(0.11)\end{array}$ & $\begin{array}{l}0.20 \\
(0.03)\end{array}$ & $\begin{array}{l}0.18 \\
(0.03)\end{array}$ & $\begin{array}{l}4.81 \\
(1.45)\end{array}$ & $\begin{array}{l}0.45 \\
(0.07)\end{array}$ & $\begin{array}{l}0.20 \\
(0.08)\end{array}$ & $\begin{array}{l}1.99 \\
(0.40)\end{array}$ & - & - & - & $\begin{array}{l}99.35 \\
(0.80)\end{array}$ \\
\hline E_ 5 & G & 21 & $\begin{array}{l}25.59 \\
(0.60)\end{array}$ & $\begin{array}{l}65.15 \\
(1.14)\end{array}$ & - & $\begin{array}{l}0.58 \\
(0.09)\end{array}$ & $\begin{array}{l}0.35 \\
(0.08)\end{array}$ & $\begin{array}{l}0.26 \\
(0.04)\end{array}$ & $\begin{array}{l}0.23 \\
(0.05)\end{array}$ & $\begin{array}{l}5.03 \\
(0.60)\end{array}$ & $\begin{array}{l}0.51 \\
(0.07)\end{array}$ & $\begin{array}{l}0.24 \\
(0.08)\end{array}$ & $\begin{array}{l}1.92 \\
(0.30)\end{array}$ & - & - & - & $\begin{array}{l}99.84 \\
(1.02)\end{array}$ \\
\hline E_6 & G & 9 & $\begin{array}{l}23.80 \\
(1.41)\end{array}$ & $\begin{array}{l}66.68 \\
(0.97)\end{array}$ & - & $\begin{array}{l}0.02 \\
(0.13)\end{array}$ & $\begin{array}{l}0.19 \\
(0.07)\end{array}$ & $\begin{array}{l}0.38 \\
(0.08)\end{array}$ & $\begin{array}{l}0.20 \\
(0.05)\end{array}$ & $\begin{array}{l}3.48 \\
(0.40)\end{array}$ & $\begin{array}{l}0.93 \\
(0.63)\end{array}$ & $\begin{array}{l}0.24 \\
(0.07)\end{array}$ & $\begin{array}{l}3.66 \\
(0.45)\end{array}$ & - & - & - & $\begin{array}{l}99.37 \\
(0.74)\end{array}$ \\
\hline E_6 & $\mathrm{Y}$ & 9 & $\begin{array}{l}22.72 \\
(1.42)\end{array}$ & $\begin{array}{l}70.21 \\
(1.47)\end{array}$ & - & $\begin{array}{l}0.07 \\
(0.08)\end{array}$ & $\begin{array}{l}0.24 \\
(0.04)\end{array}$ & $\begin{array}{l}0.24 \\
(0.04)\end{array}$ & $\begin{array}{l}0.17 \\
(0.05)\end{array}$ & $\begin{array}{l}3.26 \\
(0.53)\end{array}$ & $\begin{array}{l}2.21 \\
(0.21)\end{array}$ & $\begin{array}{l}0.23 \\
(0.07)\end{array}$ & $\begin{array}{l}0.10 \\
(0.08)\end{array}$ & - & - & - & $\begin{array}{l}99.44 \\
(0.76)\end{array}$ \\
\hline E_8 & $\mathrm{G}$ & 8 & $\begin{array}{l}25.43 \\
(0.80)\end{array}$ & $\begin{array}{l}61.61 \\
(0.52)\end{array}$ & $\begin{array}{l}1.11 \\
(0.19)\end{array}$ & $\begin{array}{l}0.10 \\
(0.03)\end{array}$ & $\begin{array}{l}0.24 \\
(0.06)\end{array}$ & $\begin{array}{l}0.53 \\
(0.11)\end{array}$ & $\begin{array}{l}0.26 \\
(0.04)\end{array}$ & $\begin{array}{l}4.50 \\
(0.29)\end{array}$ & $\begin{array}{l}1.49 \\
(0.12)\end{array}$ & $\begin{array}{l}0.20 \\
(0.03)\end{array}$ & $\begin{array}{l}5.79 \\
(0.42)\end{array}$ & - & - & - & $\begin{array}{l}100.57 \\
(1.09)\end{array}$ \\
\hline E_9 & G & 11 & $\begin{array}{l}27.92 \\
(0.78)\end{array}$ & $\begin{array}{l}63.81 \\
(1.08)\end{array}$ & - & $\begin{array}{l}0.14 \\
(0.18)\end{array}$ & $\begin{array}{l}0.28 \\
(0.04)\end{array}$ & $\begin{array}{l}0.27 \\
(0.07)\end{array}$ & $\begin{array}{l}0.16 \\
(0.06)\end{array}$ & $\begin{array}{l}4.56 \\
(1.22)\end{array}$ & $\begin{array}{l}0.56 \\
(0.10)\end{array}$ & $\begin{array}{l}0.22 \\
(0.06)\end{array}$ & $\begin{array}{l}2.21 \\
(0.37)\end{array}$ & - & - & - & $\begin{array}{l}100.13 \\
(0.78)\end{array}$ \\
\hline E_10 & G & 13 & $\begin{array}{l}23.28 \\
(0.74)\end{array}$ & $\begin{array}{l}67.16 \\
(1.44)\end{array}$ & $\begin{array}{l}1.30 \\
(0.88)\end{array}$ & $\begin{array}{l}0.06 \\
(0.07)\end{array}$ & $\begin{array}{l}0.23 \\
(0.05)\end{array}$ & $\begin{array}{l}0.29 \\
(0.04)\end{array}$ & $\begin{array}{l}0.26 \\
(0.04)\end{array}$ & $\begin{array}{l}3.98 \\
(0.41)\end{array}$ & $\begin{array}{l}0.47 \\
(0.06)\end{array}$ & $\begin{array}{l}0.19 \\
(0.07)\end{array}$ & $\begin{array}{l}2.94 \\
(0.49)\end{array}$ & $\begin{array}{l}0.08 \\
(0.06)\end{array}$ & - & - & $\begin{array}{l}100.04 \\
(1.42)\end{array}$ \\
\hline E_11 & G & 13 & $\begin{array}{l}25.33 \\
(0.40)\end{array}$ & $\begin{array}{l}69.16 \\
(1.05)\end{array}$ & - & $\begin{array}{l}0.01 \\
(0.06)\end{array}$ & $\begin{array}{l}0.31 \\
(0.05)\end{array}$ & $\begin{array}{l}0.16 \\
(0.03)\end{array}$ & $\begin{array}{l}0.15 \\
(0.03)\end{array}$ & $\begin{array}{l}3.83 \\
(0.80)\end{array}$ & $\begin{array}{l}0.33 \\
(0.09)\end{array}$ & $\begin{array}{l}0.16 \\
(0.06)\end{array}$ & $\begin{array}{l}1.42 \\
(0.24)\end{array}$ & - & - & - & $\begin{array}{l}100.86 \\
(0.78)\end{array}$ \\
\hline E_12 & $\mathrm{G}$ & 12 & $\begin{array}{l}31.82 \\
(1.34)\end{array}$ & $\begin{array}{l}60.99 \\
(3.00)\end{array}$ & - & $\begin{array}{l}0.50 \\
(0.29)\end{array}$ & $\begin{array}{l}0.61 \\
(0.22)\end{array}$ & $\begin{array}{l}0.36 \\
(0.03)\end{array}$ & $\begin{array}{l}0.19 \\
(0.05)\end{array}$ & $\begin{array}{l}4.22 \\
(1.62)\end{array}$ & $\begin{array}{l}0.40 \\
(0.10)\end{array}$ & $\begin{array}{l}0.26 \\
(0.08)\end{array}$ & $\begin{array}{l}1.25 \\
(0.35)\end{array}$ & - & - & - & $\begin{array}{l}100.62 \\
(1.03)\end{array}$ \\
\hline E_13 & G & 18 & $\begin{array}{l}24.45 \\
(0.87)\end{array}$ & $\begin{array}{l}67.88 \\
(0.93)\end{array}$ & $\begin{array}{l}0.30 \\
(0.10)\end{array}$ & $\begin{array}{l}0.02 \\
(0.05)\end{array}$ & $\begin{array}{l}0.26 \\
(0.05)\end{array}$ & $\begin{array}{l}0.46 \\
(0.05)\end{array}$ & $\begin{array}{l}0.15 \\
(0.04)\end{array}$ & $\begin{array}{l}2.41 \\
(0.64)\end{array}$ & $\begin{array}{l}0.47 \\
(0.08)\end{array}$ & $\begin{array}{l}0.16 \\
(0.05)\end{array}$ & $\begin{array}{l}2.86 \\
(0.34)\end{array}$ & - & - & - & $\begin{array}{l}99.41 \\
(0.69)\end{array}$ \\
\hline$F_{-} 1.602$ & $\mathrm{G}$ & 8 & $\begin{array}{l}31.35 \\
(1.53)\end{array}$ & $\begin{array}{l}60.78 \\
(1.23)\end{array}$ & - & $\begin{array}{l}0.04 \\
(0.04)\end{array}$ & $\begin{array}{l}0.32 \\
(0.03)\end{array}$ & $\begin{array}{l}0.42 \\
(0.04)\end{array}$ & $\begin{array}{l}0.35 \\
(0.07)\end{array}$ & $\begin{array}{l}4.35 \\
(0.39)\end{array}$ & $\begin{array}{l}0.69 \\
(0.29)\end{array}$ & $\begin{array}{l}0.16 \\
(0.08)\end{array}$ & $\begin{array}{l}1.56 \\
(0.18)\end{array}$ & - & - & - & $\begin{array}{l}99.93 \\
(0.61)\end{array}$ \\
\hline & $\mathrm{Y}$ & 14 & $\begin{array}{l}26.52 \\
(1.36)\end{array}$ & $\begin{array}{l}64.42 \\
(1.56)\end{array}$ & - & $\begin{array}{l}0.07 \\
(0.04)\end{array}$ & $\begin{array}{l}0.35 \\
(0.05)\end{array}$ & $\begin{array}{l}0.48 \\
(0.16)\end{array}$ & $\begin{array}{l}0.31 \\
(0.05)\end{array}$ & $\begin{array}{l}4.87 \\
(0.36)\end{array}$ & $\begin{array}{l}1.96 \\
(0.36)\end{array}$ & $\begin{array}{l}0.24 \\
(0.05)\end{array}$ & $\begin{array}{l}0.06 \\
(0.05)\end{array}$ & - & - & - & $\begin{array}{l}99.28 \\
(0.73)\end{array}$ \\
\hline F_2.589* & $\mathrm{Br}$ & 4 & $\begin{array}{l}31.54 \\
(1.11)\end{array}$ & $\begin{array}{l}49.05 \\
(0.63)\end{array}$ & $\begin{array}{l}2.13 \\
(0.43)\end{array}$ & $\begin{array}{l}0.10 \\
(0.02)\end{array}$ & $\begin{array}{l}0.53 \\
(0.06)\end{array}$ & $\begin{array}{l}0.88 \\
(0.10)\end{array}$ & $\begin{array}{l}0.45 \\
(0.03)\end{array}$ & $\begin{array}{l}5.90 \\
(0.20)\end{array}$ & $\begin{array}{l}2.40 \\
(0.23)\end{array}$ & $\begin{array}{l}0.32 \\
(0.02)\end{array}$ & $\begin{array}{l}6.06 \\
(1.06)\end{array}$ & $\begin{array}{l}0.15 \\
(0.08)\end{array}$ & - & - & $\begin{array}{l}99.51 \\
(0.50)\end{array}$ \\
\hline & G & 10 & $\begin{array}{l}30.80 \\
(2.35)\end{array}$ & $\begin{array}{l}55.23 \\
(5.43)\end{array}$ & $\begin{array}{l}1.42 \\
(1.94)\end{array}$ & $\begin{array}{l}0.13 \\
(0.31)\end{array}$ & $\begin{array}{l}0.49 \\
(0.07)\end{array}$ & $\begin{array}{l}0.55 \\
(0.28)\end{array}$ & $\begin{array}{l}0.33 \\
(0.11)\end{array}$ & $\begin{array}{l}5.95 \\
(0.52)\end{array}$ & $\begin{array}{l}0.82 \\
(0.44)\end{array}$ & $\begin{array}{l}0.35 \\
(0.08)\end{array}$ & $\begin{array}{l}3.74 \\
(0.28)\end{array}$ & $\begin{array}{l}0.19 \\
(0.12)\end{array}$ & - & - & $\begin{array}{l}99.86 \\
(0.95)\end{array}$ \\
\hline & BW & 5 & $\begin{array}{l}34.80 \\
(1.54)\end{array}$ & $\begin{array}{l}48.19 \\
(1.99)\end{array}$ & $\begin{array}{l}10.90 \\
(2.06)\end{array}$ & $\begin{array}{l}2.40 \\
(0.90)\end{array}$ & $\begin{array}{l}0.43 \\
(0.09)\end{array}$ & $\begin{array}{l}0.38 \\
(0.03)\end{array}$ & $\begin{array}{l}0.28 \\
(0.05)\end{array}$ & $\begin{array}{l}1.83 \\
(0.64)\end{array}$ & $\begin{array}{l}0.43 \\
(0.15)\end{array}$ & $\begin{array}{l}0.05 \\
(0.04)\end{array}$ & $\begin{array}{l}0.30 \\
(0.28)\end{array}$ & $\begin{array}{l}0.45 \\
(0.08)\end{array}$ & - & - & $\begin{array}{l}100.40 \\
(0.50)\end{array}$ \\
\hline F_3. 20 & $\mathrm{Y}$ & 8 & $\begin{array}{l}24.52 \\
(1.10)\end{array}$ & $\begin{array}{l}66.15 \\
(1.98)\end{array}$ & - & $\begin{array}{l}0.03 \\
(0.08)\end{array}$ & $\begin{array}{l}0.32 \\
(0.03)\end{array}$ & $\begin{array}{l}0.30 \\
(0.04)\end{array}$ & $\begin{array}{l}0.23 \\
(0.03)\end{array}$ & $\begin{array}{l}4.60 \\
(0.46)\end{array}$ & $\begin{array}{l}1.97 \\
(0.35)\end{array}$ & $\begin{array}{l}0.28 \\
(0.05)\end{array}$ & $\begin{array}{l}0.68 \\
(0.37)\end{array}$ & - & - & - & $\begin{array}{l}99.07 \\
(1.97)\end{array}$ \\
\hline & G & 15 & $\begin{array}{l}26.97 \\
(0.24)\end{array}$ & $\begin{array}{l}62.75 \\
(0.35)\end{array}$ & - & $\begin{array}{l}0.01 \\
(0.05)\end{array}$ & $\begin{array}{l}0.43 \\
(0.12)\end{array}$ & $\begin{array}{l}0.34 \\
(0.02)\end{array}$ & $\begin{array}{l}0.26 \\
(0.03)\end{array}$ & $\begin{array}{l}5.38 \\
(0.24)\end{array}$ & $\begin{array}{l}1.63 \\
(0.46)\end{array}$ & $\begin{array}{l}0.33 \\
(0.10)\end{array}$ & $\begin{array}{l}1.89 \\
(0.25)\end{array}$ & - & - & - & $\begin{array}{l}99.99 \\
(1.50)\end{array}$ \\
\hline & DG & 7 & $\begin{array}{l}27.03 \\
(1.69)\end{array}$ & $\begin{array}{l}63.05 \\
(2.03)\end{array}$ & - & $\begin{array}{l}0.03 \\
(0.09)\end{array}$ & $\begin{array}{l}0.39 \\
(0.21)\end{array}$ & $\begin{array}{l}0.34 \\
(0.06)\end{array}$ & $\begin{array}{l}0.23 \\
(0.06)\end{array}$ & $\begin{array}{l}5.38 \\
(0.70)\end{array}$ & $\begin{array}{l}1.65 \\
(0.53)\end{array}$ & $\begin{array}{l}0.34 \\
(0.10)\end{array}$ & $\begin{array}{l}2.12 \\
(0.67)\end{array}$ & - & - & - & $\begin{array}{l}100.50 \\
(0.88)\end{array}$ \\
\hline F_007 & G & 17 & 26.56 & 61.33 & - & 0.08 & 0.64 & 0.21 & 0.21 & 6.85 & 0.72 & 0.29 & 2.57 & - & - & - & 99.47 \\
\hline
\end{tabular}


Table 2 (continued)

\begin{tabular}{|c|c|c|c|c|c|c|c|c|c|c|c|c|c|c|c|c|c|c|}
\hline & Sample & $c$ & $n$ & $\mathrm{SiO}_{2}$ & $\mathrm{PbO}$ & $\mathrm{SnO}_{2}$ & $\mathrm{Na}_{2} \mathrm{O}$ & $\mathrm{K}_{2} \mathrm{O}$ & $\mathrm{CaO}$ & $\mathrm{MgO}$ & $\mathrm{Al}_{2} \mathrm{O}_{3}$ & $\mathrm{FeO}$ & $\mathrm{TiO}_{2}$ & $\mathrm{CuO}$ & $\mathrm{Sb}_{2} \mathrm{O}_{3}$ & $\mathrm{CoO}$ & $\mathrm{MnO}$ & Total \\
\hline & & & & $(0.53)$ & $(0.76)$ & & $(0.04)$ & (0.09) & (0.06) & (0.04) & (0.58) & $(0.11)$ & $(0.05)$ & $(0.41)$ & & & & $(0.89)$ \\
\hline & F_012 & $\mathrm{G}$ & 11 & 22.96 & 63.38 & - & 0.10 & 0.35 & 0.73 & 0.36 & 5.74 & 1.40 & 0.36 & 4.32 & - & - & - & 99.70 \\
\hline & & & & (1.63) & (1.02) & & $(0.06)$ & $(0.05)$ & (0.04) & $(0.05)$ & (0.19) & $(0.16)$ & $(0.06)$ & (0.98) & & & & $(0.51)$ \\
\hline & & $\mathrm{Y}$ & 11 & 21.86 & 68.52 & - & 0.14 & 0.44 & 0.31 & 0.24 & 5.99 & 0.43 & 0.13 & 0.65 & 0.96 & - & - & 99.63 \\
\hline & & & & $(0.75)$ & $(0.70)$ & & $(0.09)$ & (0.13) & $(0.10)$ & $(0.05)$ & $(0.17)$ & $(0.08)$ & $(0.04)$ & $(0.20)$ & $(0.73)$ & & & $(0.94)$ \\
\hline & F_014/1 & $\mathrm{G}$ & 23 & 27.96 & 58.29 & - & 0.08 & 0.45 & 0.80 & 0.42 & 6.98 & 1.39 & 0.37 & 2.67 & - & - & - & 99.43 \\
\hline & & & & $(2.58)$ & $(3.48)$ & & $(0.07)$ & $(0.22)$ & $(0.37)$ & (0.17) & $(0.52)$ & $(0.50)$ & $(0.04)$ & $(0.39)$ & & & & $(0.73)$ \\
\hline & F_017/1* & $\mathrm{G}$ & 5 & 31.11 & 51.69 & 1.63 & 0.10 & 0.55 & 0.64 & 0.36 & 6.01 & 2.41 & 0.26 & 5.00 & - & - & - & 99.75 \\
\hline & & & & (1.23) & $(2.42)$ & $(0.60)$ & $(0.05)$ & (0.07) & $(0.05)$ & $(0.02)$ & $(0.44)$ & (0.09) & $(0.02)$ & (0.88) & & & & (1.18) \\
\hline & & $\mathrm{Y}$ & 8 & 29.92 & 59.25 & 0.71 & 0.07 & 0.49 & 0.52 & 0.28 & 5.54 & 2.13 & 0.22 & 0.78 & 0.52 & - & - & 99.94 \\
\hline & & & & (2.92) & (4.29) & $(0.62)$ & $(0.03)$ & (0.12) & $(0.12)$ & $(0.03)$ & (1.06) & $(0.24)$ & $(0.05)$ & (0.63) & $(0.20)$ & & & (1.34) \\
\hline & F_017/2 & G & 16 & 30.49 & 59.06 & - & 0.09 & 0.31 & 0.51 & 0.26 & 5.11 & 0.60 & 0.30 & 2.94 & - & - & - & 99.68 \\
\hline & & & & $(1.30)$ & (1.13) & & $(0.06)$ & $(0.09)$ & $(0.04)$ & $(0.05)$ & $(0.98)$ & $(0.13)$ & $(0.23)$ & $(0.58)$ & & & & $(0.90)$ \\
\hline \multirow{38}{*}{$\begin{array}{c}\text { Group } \\
3\end{array}$} & CS_2016.41.2* & $\mathrm{G}$ & 3 & 32.94 & 55.54 & 6.73 & 0.75 & 0.22 & 0.47 & 0.37 & 1.52 & 0.16 & 0.04 & 0.60 & 0.41 & - & - & 99.77 \\
\hline & & & & $(1.48)$ & $(1.17)$ & $(1.02)$ & $(0.10)$ & $(0.05)$ & $(0.06)$ & $(0.04)$ & $(0.05)$ & $(0.03)$ & $(0.04)$ & $(0.10)$ & $(0.10)$ & & & $(1.55)$ \\
\hline & & DG & 3 & 37.48 & 55.11 & 0.13 & 0.42 & 0.39 & 0.52 & 0.68 & 4.29 & 0.39 & 0.12 & 0.81 & 0.05 & - & - & 99.77 \\
\hline & & & & $(2.44)$ & $(2.60)$ & $(0.40)$ & $(0.30)$ & $(0.05)$ & $(0.03)$ & $(0.06)$ & $(0.20)$ & $(0.13)$ & $(0.05)$ & $(0.26)$ & $(0.03)$ & & & $(1.55)$ \\
\hline & & $\mathrm{Y}$ & 3 & 23.58 & 69.18 & 3.45 & 0.36 & 0.14 & 0.12 & 0.20 & 1.15 & 0.19 & 0.00 & - & 2.59 & - & - & 101.02 \\
\hline & & & & (2.77) & $(5.04)$ & (2.67) & $(0.19)$ & $(0.07)$ & (0.08) & $(0.05)$ & $(0.39)$ & $(0.06)$ & $(0.04)$ & & $(1.26)$ & & & (0.09) \\
\hline & & W & 4 & 31.73 & 53.29 & 10.30 & 1.49 & 0.36 & 0.29 & 0.28 & 1.16 & 0.23 & 0.01 & 0.03 & 0.73 & - & 0.15 & 99.92 \\
\hline & & & & $(2.21)$ & (7.01) & (4.53) & $(0.53)$ & $(0.11)$ & $(0.04)$ & $(0.05)$ & $(0.18)$ & (0.09) & $(0.02)$ & (0.09) & $(0.11)$ & & & $(0.34)$ \\
\hline & CS_2016.89.3* & $\mathrm{B} 1$ & 4 & 26.39 & 49.87 & 18.27 & 2.30 & 0.37 & 0.47 & 0.29 & 0.68 & 0.41 & 0.03 & 0.02 & 0.79 & 0.33 & - & 100.10 \\
\hline & & & & $(1.56)$ & $(1.74)$ & (2.89) & $(0.18)$ & $(0.06)$ & $(0.02)$ & $(0.03)$ & $(0.04)$ & $(0.04)$ & $(0.06)$ & $(0.02)$ & $(0.13)$ & $(0.04)$ & & (1.11) \\
\hline & & $\mathrm{W}^{* *}$ & 5 & 25.11 & 54.81 & 15.30 & 1.49 & 0.31 & 0.48 & 0.23 & 0.72 & 0.33 & 0.00 & 0.03 & 0.68 & - & - & 99.48 \\
\hline & & & & (1.34) & $(3.68)$ & $(5.34)$ & $(0.05)$ & $(0.05)$ & $(0.07)$ & $(0.04)$ & $(0.11)$ & $(0.06)$ & $(0.07)$ & $(0.11)$ & $(0.32)$ & & & $(0.89)$ \\
\hline & & $\mathrm{Y}$ & 4 & 18.63 & 70.56 & 4.38 & 0.43 & 0.15 & 0.09 & 0.25 & 1.71 & 0.55 & 0.08 & 0.04 & 2.38 & - & - & 99.23 \\
\hline & & & & (1.87) & $(0.84)$ & (1.29) & $(0.12)$ & $(0.08)$ & $(0.05)$ & $(0.05)$ & $(0.45)$ & $(0.05)$ & $(0.06)$ & $(0.05)$ & $(0.66)$ & & & $(0.98)$ \\
\hline & CS_2016.117.3* & G & 4 & 30.31 & 56.22 & 5.95 & 0.65 & 0.42 & 0.72 & 0.34 & 2.39 & 0.74 & 0.05 & 2.48 & 0.49 & - & - & 100.75 \\
\hline & & & & $(2.30)$ & $(2.15)$ & (1.85) & $(0.22)$ & $(0.08)$ & $(0.13)$ & $(0.02)$ & $(0.44)$ & $(0.05)$ & $(0.04)$ & $(0.42)$ & $(0.16)$ & & & $(0.68)$ \\
\hline & & $\mathrm{Y}$ & 7 & 22.29 & 71.19 & 2.59 & 0.20 & 0.19 & 0.04 & 0.22 & 2.32 & 0.34 & 0.04 & 0.11 & 0.93 & - & - & 99.72 \\
\hline & & & & (3.57) & $(1.71)$ & (2.91) & $(0.06)$ & $(0.14)$ & $(0.12)$ & $(0.07)$ & $(0.92)$ & $(0.06)$ & $(0.07)$ & $(0.11)$ & (0.09) & & & $(0.97)$ \\
\hline & & W & 3 & 30.27 & 46.68 & 15.40 & 3.42 & 0.54 & 0.61 & 0.28 & 1.12 & 0.47 & 0.02 & 0.00 & 0.65 & - & - & 99.43 \\
\hline & & & & $(0.55)$ & $(0.41)$ & $(0.35)$ & $(0.37)$ & $(0.04)$ & $(0.08)$ & $(0.02)$ & $(0.18)$ & $(0.10)$ & $(0.04)$ & $(0.04)$ & (0.09) & & & $(0.17)$ \\
\hline & $\mathrm{CS}$ & G & 8 & 30.75 & 58.58 & 1.67 & 0.37 & 0.82 & 0.70 & 0.40 & 3.06 & 0.76 & 0.19 & 2.34 & 0.20 & - & - & 99.84 \\
\hline & $\overline{2016.163 .12 *}$ & & & $(1.15)$ & $(2.53)$ & (1.32) & $(0.28)$ & $(0.12)$ & $(0.15)$ & $(0.04)$ & $(0.46)$ & $(0.26)$ & $(0.08)$ & $(0.53)$ & $(0.12)$ & & & $(0.56)$ \\
\hline & & W & 6 & 35.76 & 45.11 & 10.89 & 1.74 & 1.33 & 0.66 & 0.35 & 2.17 & 0.59 & 0.03 & 0.70 & 0.61 & - & - & 99.92 \\
\hline & & & & (3.31) & $(3.71)$ & (0.67) & $(0.73)$ & $(0.35)$ & $(0.13)$ & $(0.07)$ & $(0.17)$ & $(0.11)$ & $(0.05)$ & $(0.65)$ & (0.13) & & & $(0.68)$ \\
\hline & CS_2016.204.1* & G & 9 & 31.09 & 60.10 & - & 0.12 & 0.44 & 0.71 & 0.30 & 3.37 & 0.46 & 0.11 & 2.55 & - & - & - & 99.23 \\
\hline & & & & $(0.80)$ & (1.64) & & $(0.10)$ & $(0.10)$ & $(0.06)$ & $(0.07)$ & $(0.71)$ & $(0.09)$ & $(0.03)$ & $(0.51)$ & & & & $(0.94)$ \\
\hline & & $\mathrm{Y}$ & 8 & 22.02 & 67.46 & 3.06 & 0.22 & 0.30 & 0.08 & 0.24 & 3.32 & 0.33 & 0.14 & 0.05 & 2.31 & - & - & 99.45 \\
\hline & & & & $(0.95)$ & $(0.17)$ & $(0.87)$ & $(0.03)$ & $(0.08)$ & $(0.04)$ & $(0.05)$ & $(0.42)$ & $(0.02)$ & $(0.02)$ & & $(0.68)$ & & & $(0.12)$ \\
\hline & CS_2016.211.1* & G & 2 & 24.28 & 65.96 & - & 0.53 & 0.42 & 0.62 & 0.44 & 4.34 & 1.03 & 0.14 & 2.75 & - & - & - & 100.49 \\
\hline & & & & $(0.33)$ & $(0.34)$ & & $(0.00)$ & $(0.06)$ & $(0.00)$ & $(0.01)$ & $(0.03)$ & $(0.12)$ & $(0.01)$ & $(0.58)$ & & & & $(0.04)$ \\
\hline & CS_2016.220.7 & G & 10 & 19.34 & 69.82 & - & 0.37 & 0.80 & 0.19 & 0.52 & 6.35 & 1.87 & 0.25 & 1.44 & - & - & - & 99.94 \\
\hline & & & & (1.49) & (1.32) & & (0.08) & (0.07) & (0.04) & $(0.06)$ & $(0.61)$ & $(0.12)$ & $(0.05)$ & $(0.29)$ & & & & (1.67) \\
\hline & CS_1 & G & 11 & 30.97 & 57.17 & - & 1.30 & 0.63 & 0.81 & 0.43 & 4.22 & 1.32 & 0.26 & 2.80 & - & - & - & 99.92 \\
\hline & & & & (0.17) & $(0.79)$ & & $(0.07)$ & $(0.05)$ & (0.03) & (0.06) & $(0.41)$ & $(0.14)$ & $(0.06)$ & $(0.29)$ & & & & $(0.82)$ \\
\hline & CS_2 & $\mathrm{G}$ & 23 & 33.71 & 52.76 & 0.69 & 1.04 & 1.16 & 0.55 & 0.46 & 5.51 & 1.34 & 0.26 & 2.29 & - & - & - & 99.76 \\
\hline & & & & (4.53) & $(8.82)$ & (0.19) & $(0.09)$ & (1.44) & $(0.08)$ & (0.07) & (3.94) & $(0.21)$ & $(0.03)$ & $(0.61)$ & & & & (1.04) \\
\hline & CS_3 & G & 10 & 19.76 & 68.96 & - & 0.41 & 0.34 & 0.23 & 0.52 & 6.29 & 0.80 & 0.25 & 1.40 & - & - & - & 98.96 \\
\hline & & & & $(0.43)$ & $(0.82)$ & & $(0.06)$ & $(0.25)$ & $(0.04)$ & (0.04) & (0.19) & (0.07) & $(0.05)$ & $(0.26)$ & & & & (1.13) \\
\hline \multirow[t]{2}{*}{ Outlier } & E_Green-glazed & G & 10 & 34.99 & 52.81 & - & 0.27 & 0.63 & 2.19 & 0.44 & 5.14 & 1.10 & 0.21 & 2.03 & - & - & - & 99.80 \\
\hline & grape tendrils 7 & & & (1.18) & (2.32) & & $(0.12)$ & $(0.11)$ & $(0.05)$ & $(0.06)$ & $(0.79)$ & $(0.13)$ & $(0.05)$ & $(0.53)$ & & & & $(1.05)$ \\
\hline
\end{tabular}

The italicized values in parenthesis represent the standard deviation, i.e. within \pm 1 standard deviations from the average

detected. Glazes of the polychrome tiles contain less $\mathrm{Al}_{2} \mathrm{O}_{3}$ than all the other studied tiles. In contrast, five green-glazed stove tiles ('CS 2016.211.1', 'CS 2016.220.7', 'CS 1', 'CS_2', 'CS_3') and one yellow- and green-glazed stove tile ('CS_2016.204.1') from Csábrág/Čabrad' have high- and very high-lead glazes (52.8-69.8 wt \% PbO, 0.5-1.9 wt\% $\mathrm{Na}_{2} \mathrm{O}+$ $\mathrm{K}_{2} \mathrm{O}$ ), without any tin content. For these tiles, the green glaze contains an average of 1.4-2.8 wt\% $\mathrm{CuO}$.

The green glaze of 'Green-glazed grape tendrils 7' from Eger differs from all the other tiles, because it shares some common 
characteristics with the Besztercebánya/Banská Bystrica glazes (group 1). The green glaze is high-lead type, with $53 \mathrm{wt} \% \mathrm{PbO}$, $0.9 \mathrm{wt} \%$ total alkali $\left(\mathrm{Na}_{2} \mathrm{O}+\mathrm{K}_{2} \mathrm{O}\right), \sim 2 \mathrm{wt} \% \mathrm{CaO}$, and $\sim 2 \mathrm{wt} \%$ $\mathrm{CuO}$ content (Fig. 9e-f, Table 2).

Spot and line EDS measurements from the ceramic bodyglaze interface to the outer edge of the glaze revealed a slight, gradual depletion in $\mathrm{SiO}_{2}$ (up to $3 \mathrm{wt} \%$ ) and $\mathrm{Al}_{2} \mathrm{O}_{3}$ (up to $2 \mathrm{wt} \%$ ) concentrations, as well as a slight, gradual enrichment in $\mathrm{PbO}$ content (up to $4 \mathrm{wt} \%$ ) in all the examined tiles.

\section{Microstructure and colourants}

All the glazes are single-layered, homogenous and thin (up to $200 \mu \mathrm{m}$ in thickness) layers with cracks, and a few large (up to $100 \mu \mathrm{m}$ ) bubbles (Figs. 7 and 9).

In the green glazes of Besztercebánya/Banská Bystrica, angular and rhombohedral-like lead- and antimony-bearing crystals up to $20-\mu \mathrm{m}$ size occur sparsely (except in tiles 'BB_6549' and 'BB_6555' tiles). The chemical composition of these lead antimonate crystals varies between 52.3 and $63.4 \mathrm{wt} \% \mathrm{PbO}$ and 17.0 and $35.5 \mathrm{wt} \% \mathrm{Sb}_{2} \mathrm{O}_{3}$, respectively. Furthermore, the crystals contain more iron (2.15$3.6 \mathrm{wt} \% \mathrm{FeO}$ ) than the iron content of the vitreous matrix (0.5-1.2 wt\% FeO). The Raman microspectroscopic analysis indicates that lead antimonate crystals have characteristic bands at 67.5-71, (128), 137-141, 287-298, 320-329, 446-459, and 506-510 $\mathrm{cm}^{-1}$, with some additional bands compared to the spectra of the natural mineral bindheimite $\left(\mathrm{Pb}_{2} \mathrm{Sb}_{2} \mathrm{O}_{6} \mathrm{O}\right)$ (Fig. 10a). One tile ('BB_3591') also contains lead- and antimony-bearing crystals of the same size and shape. Their chemical composition, however, is different, with high amounts of $\mathrm{SnO}_{2}(62 \pm 1 \mathrm{wt} \% \mathrm{PbO}, 19 \pm$ $2.2 \mathrm{wt} \% \mathrm{Sb}_{2} \mathrm{O}_{5}, 11.7 \pm 1.8 \mathrm{wt} \% \mathrm{SnO}_{2}$ ) indicating lead-tin antimonate (see below).

One tile from Eger ('E_1') also contains sporadic angular lead antimonate crystals, which are less than $15 \mu \mathrm{m}$ in size, but in the yellow, rather than in the green glaze (Fig. 7c). The chemical composition of the lead antimonate crystals is $62-$ $65 \mathrm{wt} \% \mathrm{PbO}, 23.5-28 \mathrm{wt} \% \mathrm{Sb}_{2} \mathrm{O}_{3}$, and $1.9-3 \mathrm{wt} \% \mathrm{FeO}$, where the $\mathrm{FeO}$ content in the lead antimonate crystals exceeds that of $\mathrm{FeO}$ in the glaze $(0.5 \mathrm{wt} \%)$. The Raman spectrum of the lead antimonate crystal in the ' $E \_1$ ' glaze is the same as that of the lead antimonate crystals in the Besztercebánya/Banská Bystrica (group 1) glazes (Fig. 10a).

In the green glaze of the monochrome tile 'E_10', lead-, tin-, and antimony-bearing aggregates (62.4-66.7 wt\% $\mathrm{PbO}$, $15-25.5 \mathrm{wt} \% \mathrm{SnO}_{2}, 0-3.35 \mathrm{wt} \% \mathrm{Sb}_{2} \mathrm{O}_{3}$ ) up to $60 \mu \mathrm{m}$ in size occur sparsely. They exhibit a different Raman spectrum compared to the lead antimonate particles present in the glazes of the 'E_1' tile and the Besztercebánya/Banská Bystrica tiles (Fig. 10b, see below).

Two tiles from Fülek also contain angular lead-, tin-, and antimony-bearing crystals less than $10 \mu \mathrm{m}$ in size in the yellow glaze ('F_012') and in the green and yellow glazes ('F $017 / 1$ '). The shape and size of these crystals are similar to those of the lead antimonate crystals in the yellow glaze of the aforementioned ' $\mathrm{E} \_1$ ', but the chemical composition is different (60.6-61 wt \% PbO, 3.7-9.7 wt\% $\mathrm{SnO}_{2}, 21-$ $\left.21.4 \mathrm{wt} \% \mathrm{Sb}_{2} \mathrm{O}_{3}, 2.15 \mathrm{wt} \% \mathrm{FeO}\right)$. Rounded tin oxide particles were detected in the green and yellow glazes of the ' $\mathrm{F} \_017 / 1$ ' tile, as well (Fig. 7f).

The green glaze of one of the yellow- and green-glazed polychrome tiles from Eger ('E_2') has a relatively high copper content $(5.6 \mathrm{wt} \% \mathrm{CuO})$, accompanied by the presence of newly formed, 10-30 $\mu \mathrm{m}$ long, tabular copper-rich crystals (82-97 wt\% CuO) (Fig. 7d). Raman microspectroscopy showed that the crystals are tenorite $(\mathrm{CuO})$.

The light brownish-white glaze of ' $\mathrm{F}$ 2.589' tile contains rounded tin oxide (Raman microspectroscopy detects cassiterite) crystals that are less than $5 \mu \mathrm{m}$ in size, as well as subrounded quartz particles (up to $90 \mu \mathrm{m}$ ). Needle-like copperrich crystals $(<10 \mu \mathrm{m})$ appear sporadically in the green and brown glazes.

The polychrome glazes of the tiles from Csábrág/Čabrad' (except 'CS_2016.204.1') contain varying amounts of tin oxide (cassiterite) particles (Fig. 9a) that present as less than $10-\mu \mathrm{m}$-sized inclusions and aggregates up to $50 \mu \mathrm{m}$ in size. Additionally, undissolved, sub-angular, $10-100-\mu \mathrm{m}$-sized mineral particles (mainly quartz and in lower amounts, Kfeldspar) occur in some of the green, yellow, white, and blue glazes (Fig. 9d).

Diverse, mostly rounded, heterogeneous lead-, tin-, and antimony-bearing grain aggregates up to $30 \mu \mathrm{m}$ in size occur in all the yellow glazes of the tiles from Csábrág/Čabrad' (61.5-66 wt\% PbO, 3.7-24 wt\% $\mathrm{SnO}_{2}, 4.5-11$ wt $\% \mathrm{Sb}_{2} \mathrm{O}_{3}$ ) (Fig. 9b, c). The Raman spectra of these lead-tin antimonate aggregates are similar to that of the particles found in the glaze of the ' $\mathrm{E} \_10$ ' tile, with characteristic bands at 66-69, 133137, (251-260), 318-329, (359), 443-457, (490-499) $\mathrm{cm}^{-1}$ (Fig. 10b).

\section{Ceramic body-glaze interface}

Newly formed crystals developed at the ceramic body-glaze interface. This interface layer has a variable thickness up to $40 \mu \mathrm{m}$, depending on the samples investigated. Crystals with size smaller than $10 \mu \mathrm{m}$ are nucleated at the interface in most of the samples. Several crystals, however, are as long as $35 \mu \mathrm{m}$ (e.g. 'BB_St. Peter' (Fig. 7a), 'E_2' (Fig. 7d), 'SZ_Ornamental'). Tiles from Csábrág/Čabrad' have the thinnest body-glaze interface layer (Fig. 9a-d). Newly formed crystals are mostly tabular and needle-like lead feldspars with various chemical compositions including potassium (22$44.6 \mathrm{wt} \% \mathrm{PbO}, 32-49 \mathrm{wt} \% \mathrm{SiO}_{2}, 17-26 \mathrm{wt} \% \mathrm{Al}_{2} \mathrm{O}_{3}, 2.5$ $\left.8.6 \mathrm{wt} \% \mathrm{~K}_{2} \mathrm{O}\right)$. Calcium silicate crystals $(12-35 \mathrm{wt} \% \mathrm{CaO}$, 4.5-15 wt $\% \mathrm{Al}_{2} \mathrm{O}_{3}, 40-45 \mathrm{wt} \% \mathrm{SiO}_{2}$ ) developed in four tiles 
Fig. 7 Microstructure and inclusions of groups 1 and 2 glazes (BSE images). a, b Greenglazed tiles from Besztercebánya/ Banská Bystrica with lead antimonate particles and newly formed lead feldspar and Ca silicate crystals-'BB_St. Peter', 'BB_6547'. c Yellow glaze of 'E 1 '' with lead antimonate colourant particles. d Green glaze of 'E_2' with newly formed copper oxide (tenorite) crystals. e Green glaze of 'F_2.589' with tin oxide particles and newly formed copper oxide and lead feldspar crystals. f Yellow glaze of ' $F$ 017/1' with tin oxide and lead-tin antimonate particles. Newly formed lead feldspar crystals occur at the ceramic body-glaze interface in all tiles

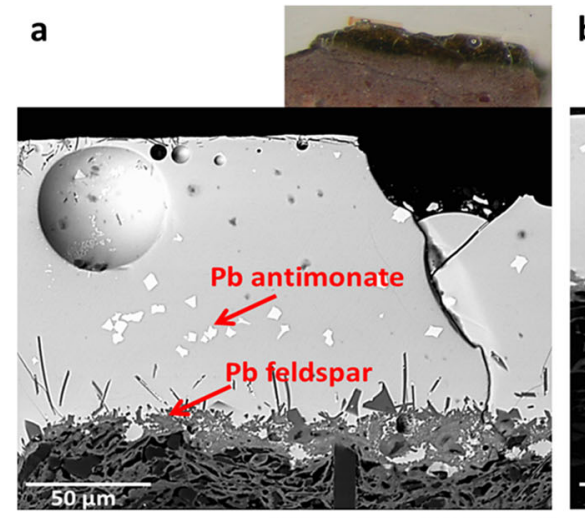

b

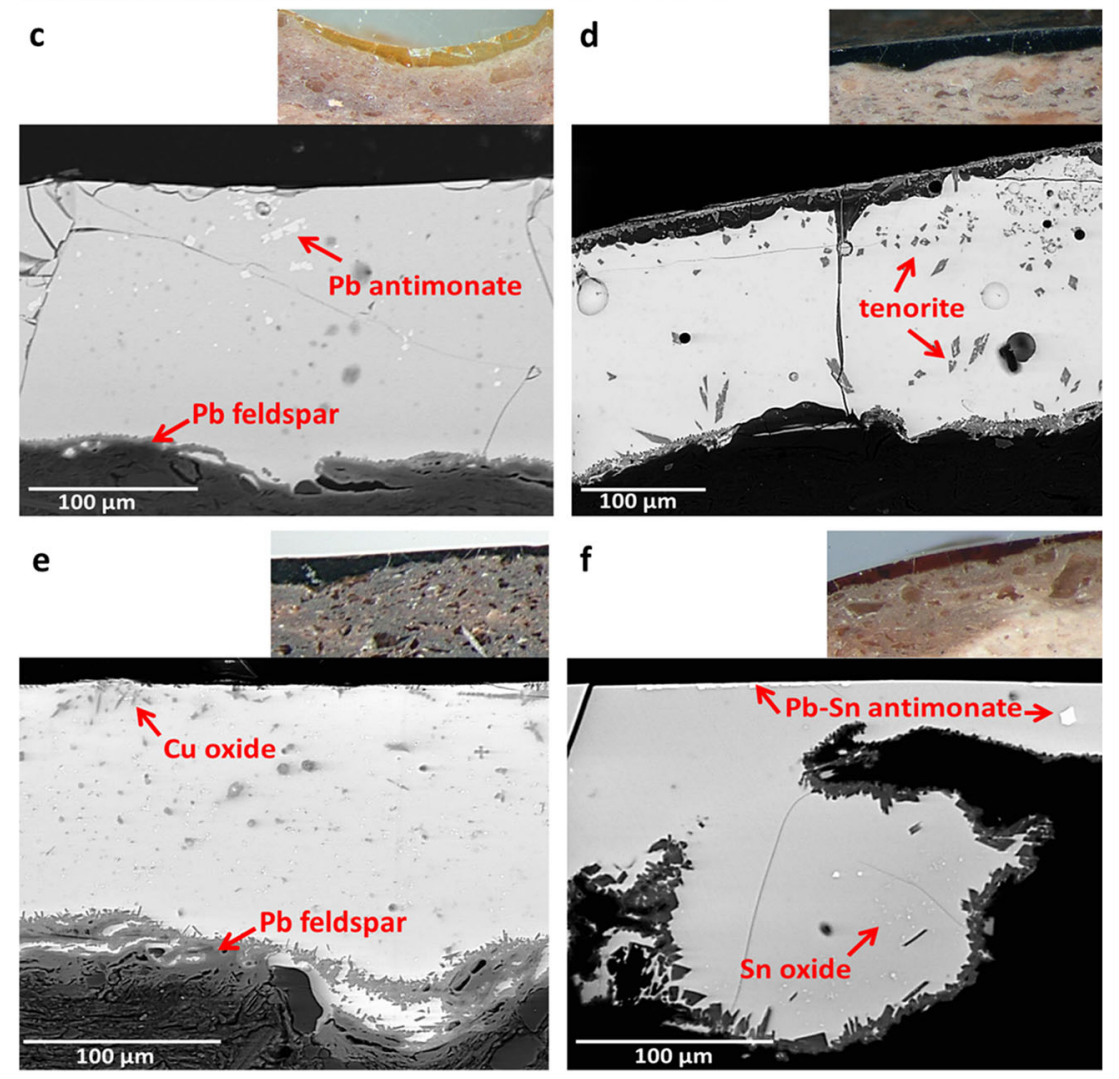

from Besztercebánya ('BB_3589a', 'BB_6547', 'BB_6554', BB_6557'), as well (Fig. 7b). Furthermore, the unique tile from Eger ('E_7') contains newly formed tabular magnesium-calcium silicates $(3.5-25$ wt $\% \mathrm{CaO}, 12.5-$ 47 wt $\% \mathrm{MgO}, 2.5-10$ wt $\% \mathrm{Al}_{2} \mathrm{O}_{3}, 39-50.3 \mathrm{wt} \% \mathrm{SiO}_{2}$, Fig. 9f) up to $15 \mu \mathrm{m}$ (detected as diopside by Raman microspectroscopy) and calcium-iron aluminosilicates (1530 wt $\% \mathrm{CaO}, 2.3-12 \mathrm{wt} \% \mathrm{FeO}, 25.5-28.5$ wt $\% \mathrm{Al}_{2} \mathrm{O}_{3}$, $34.4-40.4 \mathrm{wt} \% \mathrm{SiO}_{2}$ ) in addition to the lead feldspar crystals.

In the case of six tiles from Csábrág/Čabrad' (five are polychrome tiles_'CS_2016.41.2', 'CS_2016.89.3', 'CS_2016.117.3', 'CS 2016.163.12', 'CS_2016.204.1'—and one is a monochrome green-glazed tile-'CS_2016.211.1') displayed diffusion of lead into the top of the ceramic body (with a maximum depth of $100 \mu \mathrm{m})$ (Fig. 9c).

\section{Discussion}

\section{Ceramic body}

Petrographic features and the phase composition of the ceramic body of the 'Besztercebánya/Banská Bystrica-type' stove tiles indicate that all the tiles were made from non-calcareous illitic clay. However, the mullite phase detected in the ceramic body of all samples from Szécsény and in some samples from Salgó (group 2) (Györkös et al. 2018, in press) may possibly indicate the use of illitic-kaolinitic clay as a raw material. Several studies document the formation of mullite in ceramics as a result of increasing temperature. Mullite formation is affected not only by temperature but also by the composition of the raw material as well as by the duration of the heating 


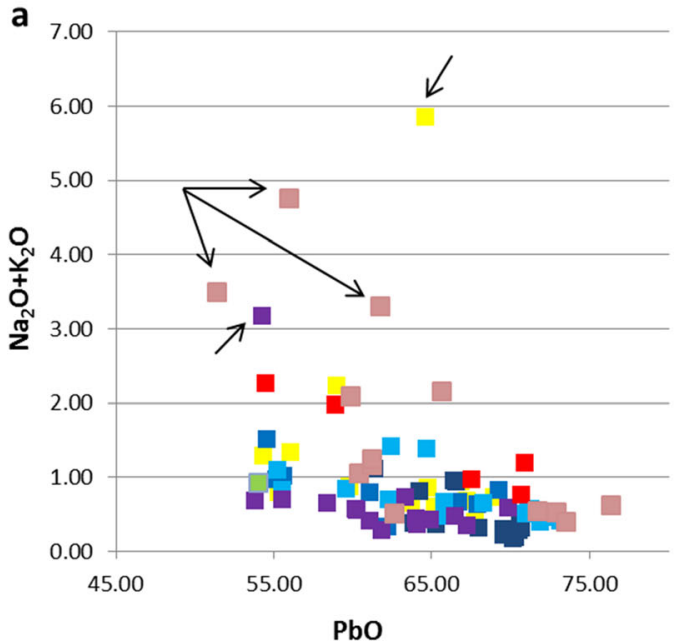

Fig. 8 Chemical composition of the glazes from each site. a $\mathrm{Na}_{2} \mathrm{O}+\mathrm{K}_{2} \mathrm{O}$ versus $\mathrm{PbO}$. b $\mathrm{Na}_{2} \mathrm{O}+\mathrm{K}_{2} \mathrm{O}$ versus $\mathrm{Al}_{2} \mathrm{O}_{3}$ after subtracting the glaze colourants and opacifiers. Glazes marked with arrows are BB_Adam

and cooling events. Mullite forms at temperatures above $1100{ }^{\circ} \mathrm{C}$ from pure kaolinite (Heimann 2010). However, if $\mathrm{K}$-feldspar, plagioclase, or quartz are present, mullite begins to grow at a lower temperature, at about $900-1100{ }^{\circ} \mathrm{C}$ (Heimann 2010). Mullite forms while mica-rich clays are fired, due to the transformation of muscovite/illite to mullite at about $900-1000{ }^{\circ} \mathrm{C}$ (Cultrone et al. 2001, 2014). If alkali and alkaline earth ions as well as water are present, then mullite starts to form at lower temperatures, i.e. at $800-900{ }^{\circ} \mathrm{C}$ (Rodriguez-Navarro et al. 2003). In this study, EPMA-WDS measurements confirmed the presence of potassium (up to $5 \mathrm{wt} \% \mathrm{~K}_{2} \mathrm{O}$ ) in the plastic matrix of the ceramic body. There is, however, no difference in the chemical composition of the matrix between the ceramic bodies that contain mullite ('E_2', 'SZ_Ornamental', 'SZ_Coat of arms 1') and those that lack mullite (Fig. 5). The chemical differences detected in the matrix of the ceramic bodies (e.g. most of the tiles from Salgó, Eger, Szécsény, and Fülek/Fil'akovo were enriched in alumina compared to the tiles of Besztercebánya/Banská Bystrica and Csábrág/Čabrad') are rather related to the different clayey raw materials used. Thus, we conclude that the raw material is illitic clay in all cases and that the presence of mullite in some of the bodies is due to the different firing conditions, such as temperature.

The presence of the mullite phase in the subgroups $2 \mathrm{~A}$ and $2 \mathrm{~B}$ tiles indicates that they reached $850{ }^{\circ} \mathrm{C}$ firing temperatures (Heimann 2010; Rodriguez-Navarro et al. 2003; Cultrone et al. 2001, 2014; Quinn 2013). Tiles (group 1, subgroup $2 \mathrm{C}$, and E_7) containing primary calcite and carbonate rock fragments (with reaction rims), detected by petrography and XRD, imply a lower firing temperature, between 650 and $700{ }^{\circ} \mathrm{C}$ (Maggetti 1982; Cultrone et al. 2001). This temperature range is also supported by the absence of other newly formed high-temperature (silicate) phases. The presence of illite and carbonate (in small amount), as well as the lack of newly formed high-temperature minerals, indicates that the maximum firing temperature of group 3 is $650-700{ }^{\circ} \mathrm{C}$.

Petrography showed that most of the analysed stove tiles from Salgó, Eger, Szécsény, and Fülek/Fil'akovo contain large quantities of mainly angular, coarse, and very coarse sandsized, non-plastic inclusions (primarily granitoid and quartzite rock fragments), which is evidence of intentional tempering. The presence of argillaceous rock fragments and the absence of coarse sand-sized fragments in some samples (subgroups $2 \mathrm{~B}$ and $2 \mathrm{C}$ ) suggest that clay mixing was used for those bodies. The Besztercebánya/Banská Bystrica tiles characterised by the presence of sub-angular magmatic, metamorphic, and calcareous rock fragments in the matrix, which may be original components of the raw material, or alternatively, may indicate intentional tempering as well. Ceramic bodies of the unglazed Bothár house (BB_52.3757.1) stove tile and its negative from the Ébner house (BB_52.3758.1) are also very similar, indicating that both the tile and the negative, although found in different parts of the same city, were produced from the same raw material, and likely in the same workshop. The ceramic body of the Csábrág/Čabrad' tiles forms an independent group (group 3), containing large, rounded (in some cases sub-angular), hydrothermally altered volcanic rock fragments most probably as temper.

\section{Glaze}

\section{Preparation technique of the glazes applied to the tiles}

Microchemical and microstructural analyses of most of the examined glazes show they are single-layered, transparent, high-lead, and very high-lead glazes (majority of the tiles from Besztercebánya/Banská Bystrica, Eger, Salgó, Szécsény, and 
Fig. 9 Microstructure and inclusions of group3 glazes and of the green glaze of an outlier (BSE images). a, b Tin oxide and leadtin antimonate inclusions in the white ('CS_2016.117.3') and yellow glazes ('CS_2016.204.1') of tiles from Csábrág/Čabrad'. c Lead-tin antimonate inclusions in the yellow glaze (' $\mathrm{CS}$

2016.117.3') and diffusion of lead into the top of the ceramic body. $\mathbf{d}$ Undissolved quartz particles in the blue glaze ('CS_2016.89.3') of tiles from Csábrág/Čabrad'. e, $\mathbf{f}$ Green glaze of 'Eger_Greenglazed grape tendrils 7 ' tile with newly formed lead feldspar and magnesium-calcium silicate crystals (diopside)
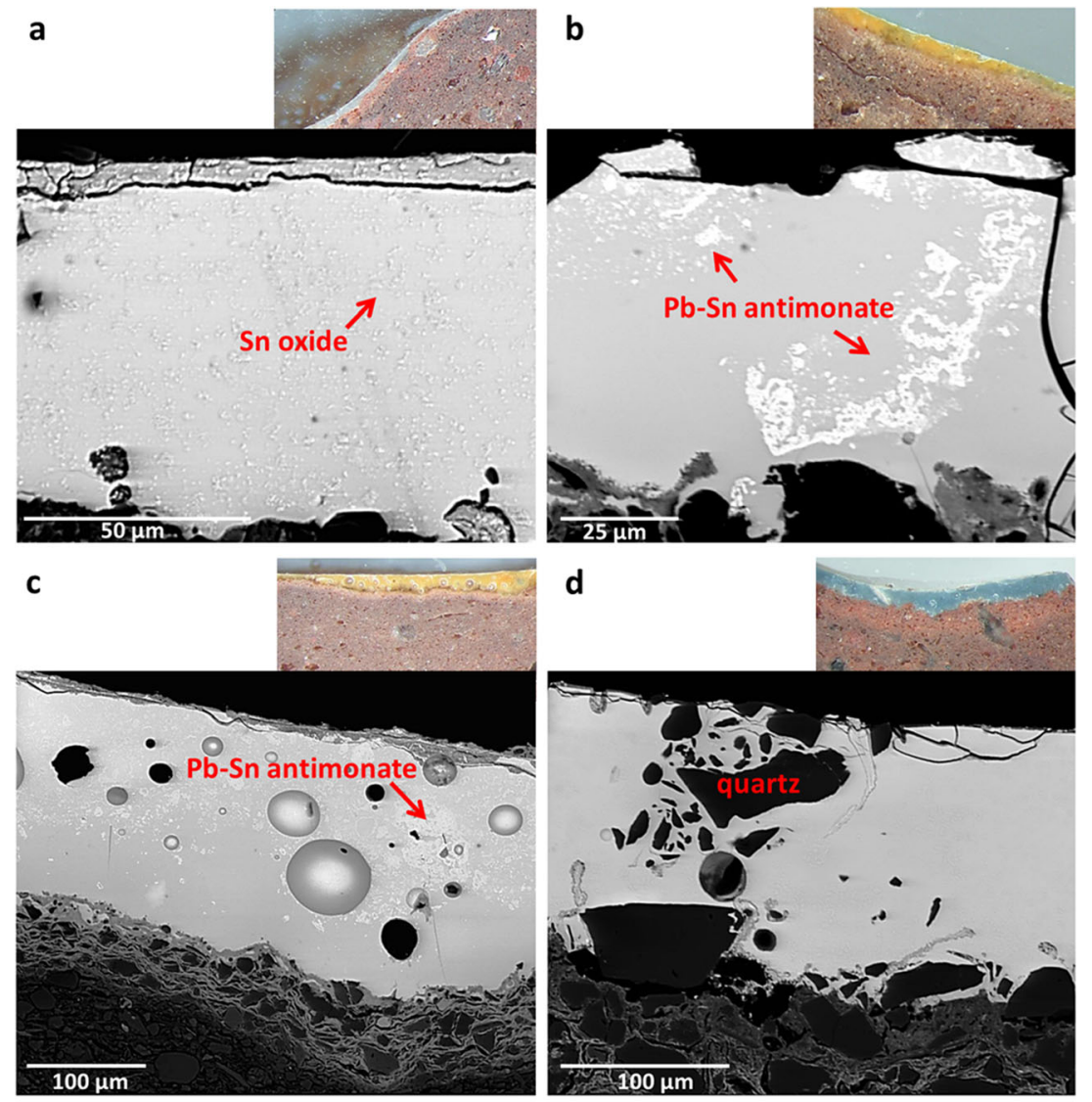

e
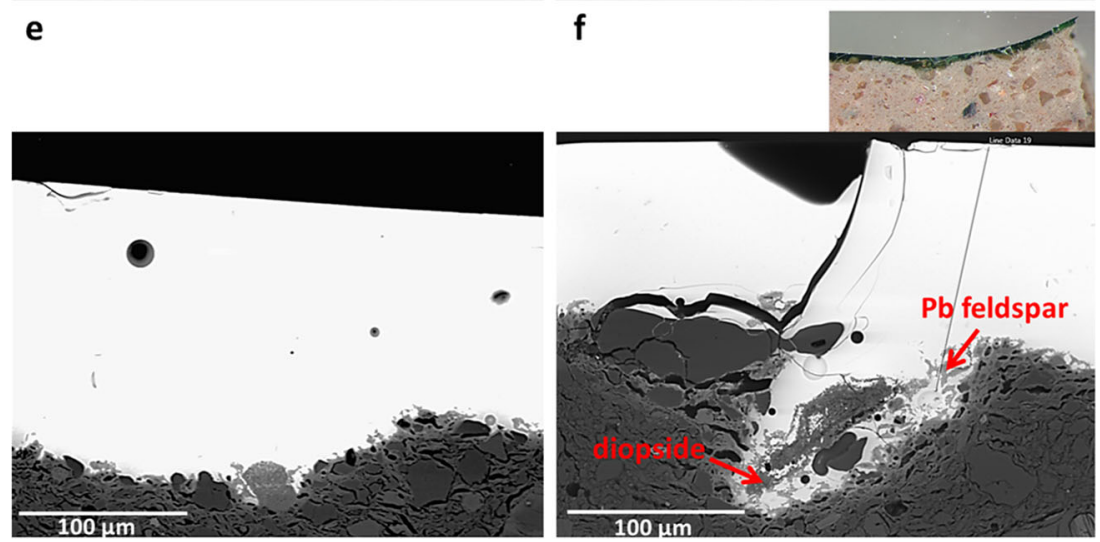

Fülek/Fil'akovo and the monochrome tiles from Csábrág/ Čabrad').

Two basic methods can be used to produce high-lead glazes (Tite et al. 1998; Walton and Tite 2010). First, a lead compound (e.g. lead oxide) can be directly applied to the ceramic body, and the glaze is formed by a reaction with the ceramic body. Second, a lead compound can be mixed with silica (quartz particles, quartz sand, or siliceous clay) and applied directly to the body, in which case, the glaze is formed directly, and then, a subsequent reaction occurs with the body. The lead compound and the silica may have been melted together first, producing a 'frit', which is then ground and applied to the ceramic body. In both methods, a small amount of clay can be added to the glaze suspension, which is then applied to a leather hard, a dry unfired, or a biscuit-fired body.

In the case of lead glazes, comparison of the ceramic body's chemical composition with that of the glaze may reveal the glaze preparation method, such as the use of lead compound by itself or mixed with silica. Using the equation proposed by Walton and Tite (2010), lead oxide and any intentional colourants or opacifiers (tin oxide, copper oxide, and antimony oxide) are subtracted from the original chemical composition of the glazes, after which the rest $\left(\mathrm{SiO}_{2}+\right.$ $\mathrm{Al}_{2} \mathrm{O}_{3}+\mathrm{Na}_{2} \mathrm{O}+\mathrm{K}_{2} \mathrm{O}+\mathrm{CaO}+\mathrm{MgO}+\mathrm{FeO}+\mathrm{TiO}_{2}$ ) is renormalised to $100 \%$ total. The renormalised $\mathrm{SiO}_{2}, \mathrm{Al}_{2} \mathrm{O}_{3}$, $\mathrm{FeO}$, and $\mathrm{CaO}$ concentrations of the glazes are compared with 


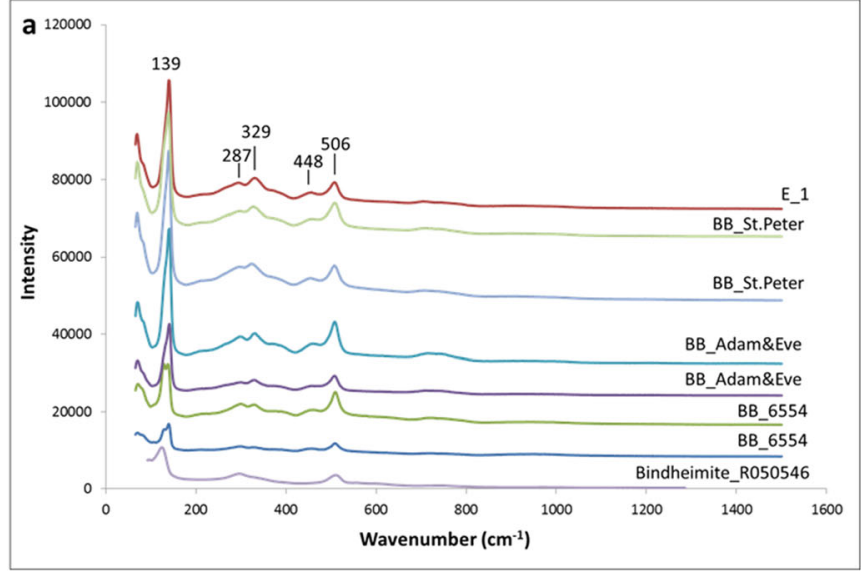

Fig. 10 Raman spectra of colourants. a Lead antimonate in the glaze of Besztercebánya/Banská Bystrica tiles (group 1) and in 'E_1' tile (group 2). Reference is bindheimite (R050546) from the RRUFF database. b

the concentrations of the similar elements in the matrix of the tile bodies (Fig. 11). The glazes contain more normalised silica than do the bodies, whereas nearly all alumina data points fall below the unity slope line. This suggests that a mixture of lead oxide and silica was used during the preparation of these glazes. The glaze also contains a higher $\mathrm{CaO}$ than does the matrix of the ceramic body, potentially indicating the use of non-calcareous clayey raw materials. Some glazes of group 2 tiles have more iron oxide than does the ceramic body, which shows that an iron colourant was intentionally added to the glaze (Fig. 11). The data points of silica and alumina of three tiles (SZ_Ornamental, SZ_Coat of arms 1, CS_3) fall on or very close to the unity slope line, suggesting that the master applied lead oxide by itself directly to the surface of the ceramic body.

Polychrome tiles from Csábrág/Čabrad' as well as three tiles from Northern Hungary ('E_2', 'F_2.589', 'F_017/1') have tinopacified high- and very high-lead glazes. During the production of tin-opacified glazes, first lead and tin metal are first melted together to form a mixture of lead-tin oxide (lead-tin calx) (Matin 2019). Then, silica, and/or alkali and/or other fluxes, can be added to the glaze suspension using various methods (such as the glaze preparation methods of the Italian maiolica and French faience, Mason and Tite 1997; Tite et al. 2008; Tite 2009; Maggetti 2012). Alkali and sand are fritted together, and then, the frit is mixed with the lead-tin calx, after which this mixture can be fritted further. Alternatively, sand, alkali, and additional lead flux are added directly to the lead-tin calx, and then, the mixture can be fritted and applied to the ceramic body. The tinopacified glazes of the tiles from Csábrág/Čabrad' and Northern Hungary are of high- and very high-lead type, with less $\mathrm{Al}_{2} \mathrm{O}_{3}$ and minimal alkali (up to $1.5 \mathrm{wt} \% \mathrm{Na}_{2} \mathrm{O}+\mathrm{K}_{2} \mathrm{O}$, except up to 4 wt $\% \mathrm{Na}_{2} \mathrm{O}+\mathrm{K}_{2} \mathrm{O}$ in white glazes). This may indicate that primarily (impure) sand and lead flux were added to the glazing mixture, instead of alkali, with the exception of white glazes, which contain a small amount of alkali. Polychrome and green

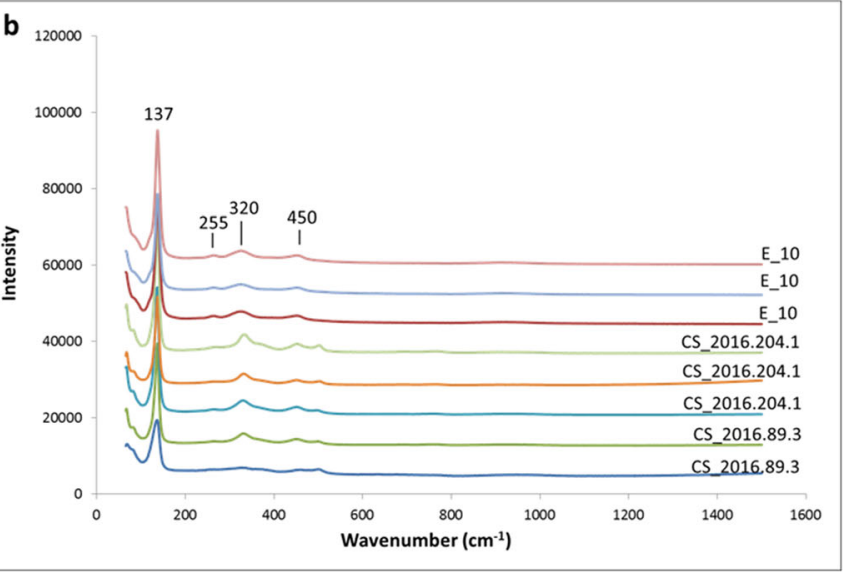

Lead-tin antimonate in the glaze of the Csábrág/Čabrad' tiles (group 3) and in 'E_10' tile (group 2) (BB, Besztercebánya/Banská Bystrica; CS, Csábrág/Čabrad'; E, Eger)

glazes of several tiles from Csábrág/Čabrad' have sub-angular-torounded quartz and K-feldspar grains in the glaze, evidencing that sand was used to prepare the glaze suspension. This suggests that the glaze suspension was not fritted prior to application on the ceramic body or that the glazing suspension was fritted, after which more sand was added to it (e.g. Italian maiolica) (Tite 2009).

Studies show that the interface layer, as a result of the reaction between the ceramic body and the glaze, is generally larger and that the concentration of newly formed crystals is generally higher when the glaze is applied to an unfired body, rather than to a biscuit-fired body. However, several other factors can influence the concentration of the crystals, such as ceramic body composition, firing temperature, duration of firing, and cooling rate (Tite et al. 1998; Molera et al. 2001; Walton and Tite 2010). Moreover, the element diffusion from the body into the glaze can also help to distinguish between the two application techniques: the diffusion is significantly greater in the unfired body than in the biscuit-fired body (e.g. Palamara et al. 2016). In our research, a considerable difference exists between the thickness of the interaction zone and the amount of newly formed crystals in group 1 and group 2 tiles and 'E_7' tiles compared to that of group 3 tiles. In group 1 , group 2, and the 'E_7' tiles, the wide (up to $40 \mu \mathrm{m}$ ) interaction zone at the ceramic body-glaze interface, where newly formed tabular lead feldspar, calcium silicate, calcium-iron aluminosilicate, or diopside crystals developed, indicates that the glaze may have been reacted with the unfired clay paste (Figs. 7 and 9e, f). In the polychrome glazes of group 3, a thin $(<10 \mu \mathrm{m})$ interaction zone with newly formed tabular lead feldspar crystals at the ceramic body-glaze interface (Fig. 9a-d), usually implies the use of a biscuit-fired body. However, the diffusion of lead into the ceramic body, in addition to the gradual diffusion of the elements from the ceramic body into the glaze suggest that the ceramic body was either unfired or lightly fired before the glaze was applied. 


\section{Glaze colourants and opacifiers}

Most of the examined stove tiles have a traditional green glaze, with different shades resulting from varying amounts of dissolved copper.

All but two tiles from Besztercebánya/Banská Bystrica are characterised by the presence of angular-rhombohedral lead antimonate crystals in the green glaze. Although the chemical composition of these crystals is not stoichiometric (58 wt\% $\mathrm{PbO}, 42 \mathrm{wt} \% \mathrm{Sb}_{2} \mathrm{O}_{5}$ with a $\mathrm{PbO} / \mathrm{Sb}_{2} \mathrm{O}_{5}$ ratio of 1.38 , Shortland 2000; Molina et al. 2014) and their Raman spectra are slightly different from that of the natural mineral bindheimite $\left(\mathrm{Pb}_{2} \mathrm{Sb}_{2} \mathrm{O}_{7}\right)$, they can be identified as the common artificial colourant Naples yellow. This colourant was a wellknown colourant for yellow glass since about $1500 \mathrm{BC}$ until the end of the Roman period (Wainwright et al. 1986; Mass et al. 2002; Shortland 2002) and was used again from the late fifteenth-early sixteenth centuries onward during glass, maiolica, and faience production, as well as in paintings (Dik et al. 2005; Tite 2009; Maggetti et al. 2009; Chiarantini et al. 2015). The earliest recipes for lead antimonate yellow were recorded by Cipriano Piccolpasso in his treatise Li tre libri dell'arte del Vasaio, written in c. 1557 (Lightbown and Caiger-Smith 2007). Various proportions of lead oxide, antimony oxides, and alkali flux, such as salt or wine lees, were mixed together and roasted (Wainwright et al. 1986; Dik et al. 2005). Iron may have been incorporated into the crystalline structure of lead antimonate. The iron content of the lead antimonate was greater than that of the vitreous matrix of the glaze covering the Besztercebánya/Banská Bystrica tiles, indicating that iron was intentionally added to the colourant. As evidenced in Piccolpasso's treatise, iron was likely added to the mixture of lead and antimony in the form of iron scale (Lightbown and Caiger-Smith 2007).

The types, chemical compositions, and production techniques of lead antimonate colourant used in both glasses and

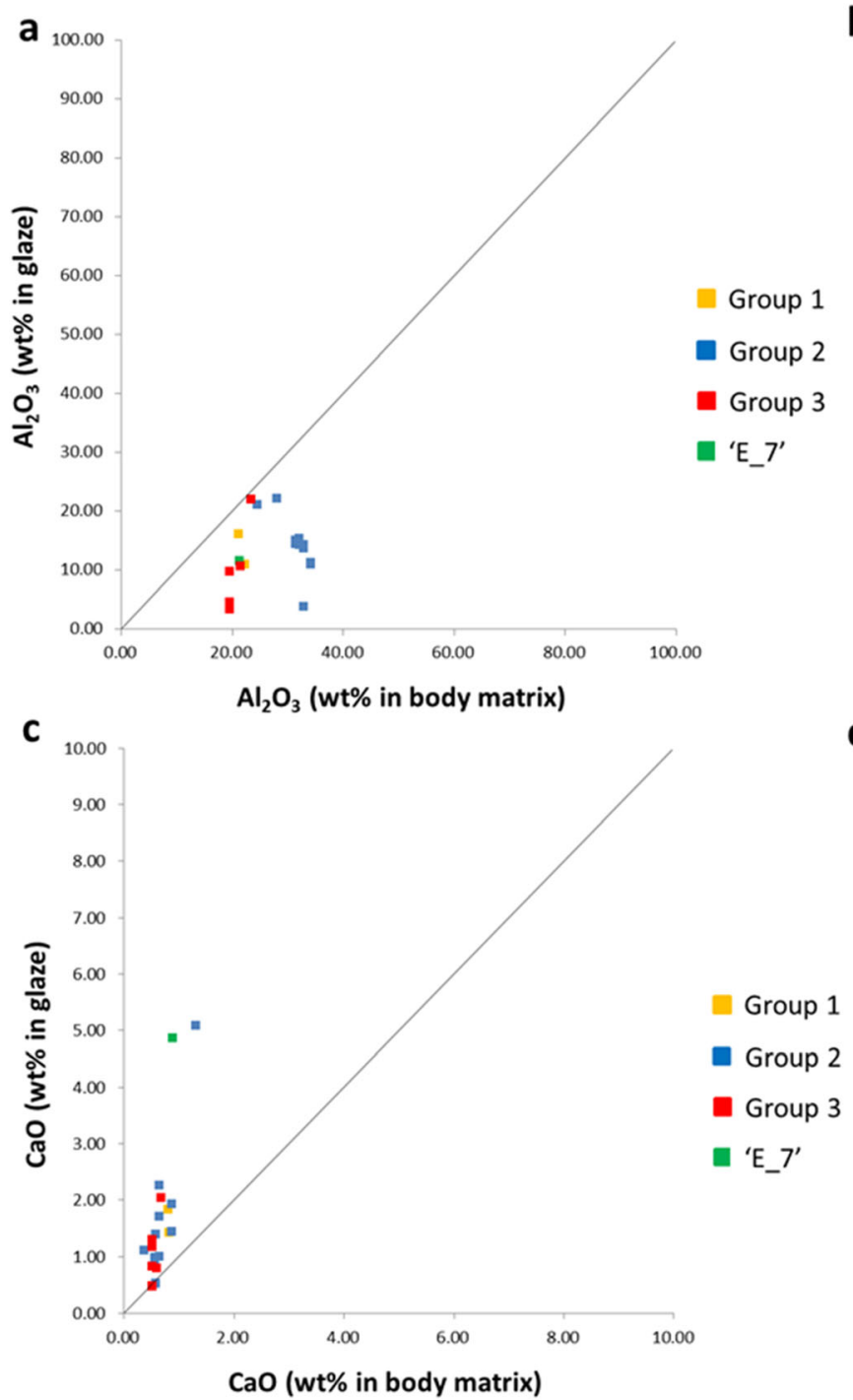

b

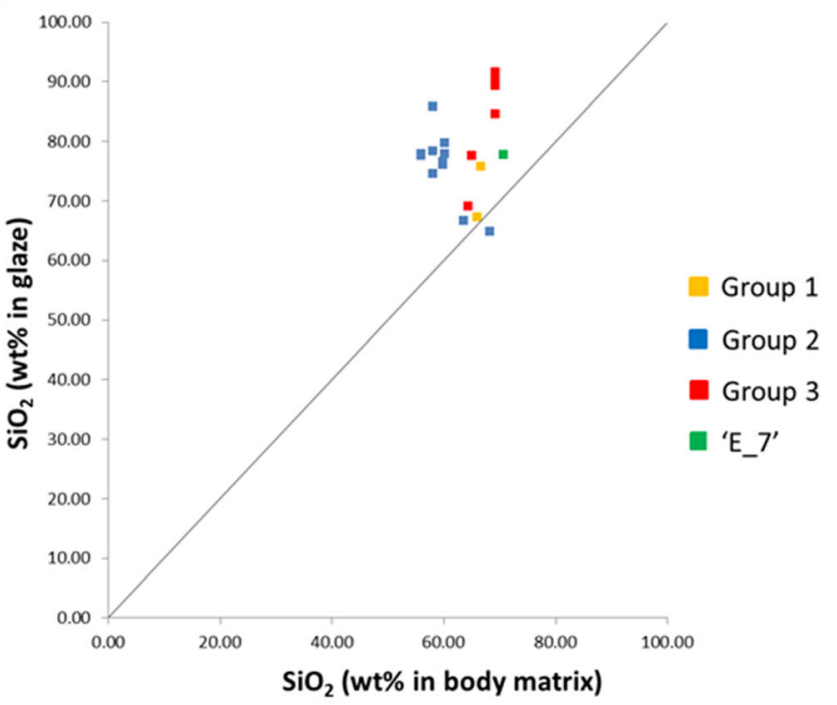

d

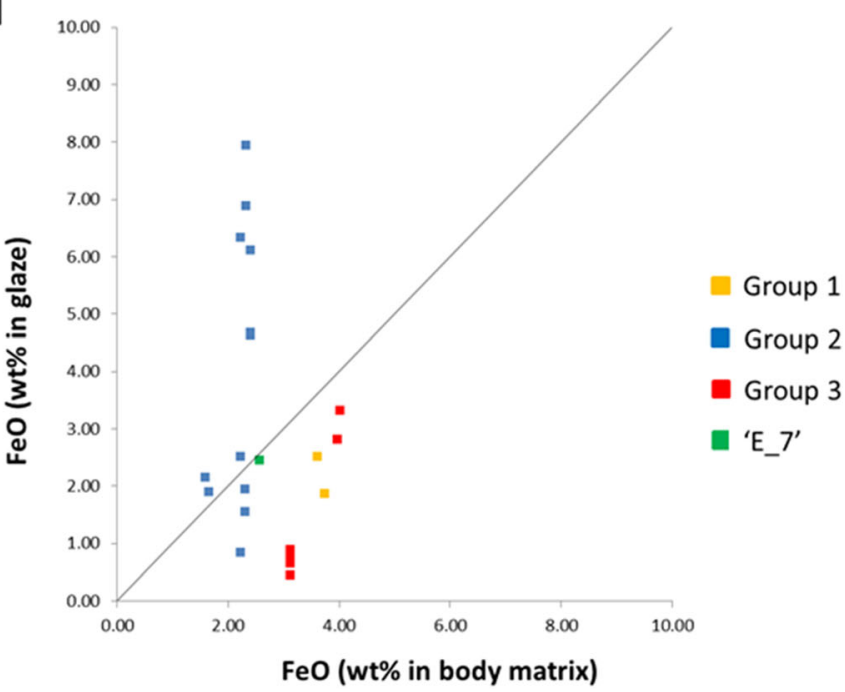

Fig. 11 Plots of normalised glaze composition vs. ceramic body composition of the same tiles for $\mathbf{a} \mathrm{Al}_{2} \mathrm{O}_{3}, \mathbf{b ~} \mathrm{SiO}_{2}, \mathbf{c ~} \mathrm{CaO}$, and $\mathbf{d ~ F e O}$ 
glazes have been researched (e.g. Mass et al. 1996, 1998; Shortland 2002; Lahlil et al. 2008; Maggetti et al. 2009; Molina et al. 2014). Based on these studies, the rhombohedral shape, small size (up to $20 \mu \mathrm{m}$ ), and rare occurrence of the lead antimonate crystals in the Besztercebánya/Banská Bystrica green glazes may be due to in situ crystallisation during firing, although lead antimonate colourant may have been intentionally added to the glaze, as well. A small amount of antimony in the lead oxide (prepared from the galena ore) may be responsible for the in situ formation of crystals. A similar conclusion (i.e. newly formed crystals were caused by impurities from the raw material) was made by Ting et al. (2019) to explain the presence of lead-antimony particles in Islamic glazes, because of their shape, rare occurrence, and small size $(<10 \mu \mathrm{m})$. Lead oxide mixed with antimony was also found by Salinas et al. (2019a) in the glaze of Islamic ceramics and was interpreted to be related to galena ores. Its appearance, however, is completely different, as it consists of small spherical precipitates. Another explanation is that lead antimonate colourant was intentionally added to the green glaze. This is supported by the higher iron content of the crystals than of the vitreous matrix of the glaze. The possibility of recrystallisation can be rejected due to the high stability of the crystals up to $1100{ }^{\circ} \mathrm{C}$ (Dik et al. 2005). Regardless, the presence of lead antimonate crystals is a defining characteristic of the Besztercebánya/Banská Bystrica tiles. The masters potentially knew that the addition of small amounts of antimonate yellow colourant to the glaze produced a fresh green colour with a yellowish tone. Piccolpasso described two types of green: 'burnt copper' and 'mixed green' in the Italian maiolica. 'Mixed green', a prefired mixture composed of copper oxide, lead oxide, and antimony oxide, was used to achieve a green colour that lacked a bluish tint (Tite 2009).

Yellow glazes from Eger are coloured with iron ('E_2', 'E_6') and, in one tile, lead antimonate ('E_1', the same as in the tiles of Besztercebánya/Banská Bystrica). For these cases, the colourants were intentionally added to the yellow glazes. For the tiles from Csábrág/Čabrad', one tile from Eger (E_10), and two tiles from Fülek/Fil'akovo ('F_012', 'F_017/ $\left.1^{\prime}\right)$, the yellow colourant is lead-tin antimonate, in the form of grain aggregates. Lead-tin antimonate was not the most common colourant for yellow glazes, although it was found in sixteenth century Italian maiolica and nineteenth century French faience glazes (Sandalinas et al. 2006; Rosi et al. 2011; Maggetti et al. 2009). The pyrochlore structure of lead-tin antimonate resembles the prevalent yellow colourants, lead-tin yellow types I and II (lead stannate), and lead antimonate, which may be the reason why this colourant was not identified until the middle of the twentieth century (Sandalinas and Ruiz-Moreno 2004). Another challenge is that tin from the glaze can be incorporated into the pyrochlore structure of lead antimonate as well, making it difficult to separate the two colourants. The earliest recipes for lead-tin antimonate are connected to the Murano glass industry. Most of the recipes entail the mixing of lead, tin, antimony, and silica together (Sandalinas and Ruiz-Moreno 2004). In our samples, the shape, size, and Raman spectra of the lead-tin antimonate aggregates in the yellow glaze of the Csábrág/ Čabrad' tiles and in the green glaze of one Eger ('E_10') tile differ significantly to the Raman spectra of the aforementioned lead antimonate crystals. Thus, the colourant was intentionally added to the glaze. The precise method, however, is less certain. One possibility is that the yellow colourant was synthesised using lead-, tin-, and antimony-bearing compounds, and was then added to the glaze. Another possibility is that the reaction between lead antimonate and tin oxide particles in the glaze resulted in the incorporation of the tin into the colourant structure (Pradell et al. 2013). The irregular shape of the aggregates suggests the first method, i.e. the intentional addition of a lead-tin antimonate colourant.

Tin oxide (in green, white, and blue glazes) or lead-tin antimonate (in yellow glazes) was detected in the polychrome glazes of the Csábrág/Čabrad' tiles. This suggests they were intentionally added as opacifiers. The light blue colour was achieved with a cobalt colourant in the tin-opacified glaze.

\section{Distinction between workshop products}

Our analyses show that the raw materials, production technologies, and recipes of the ceramic bodies and glazes of the stove tiles differed between sites (Table 3).

The macroscopic appearance, as well as the chemical composition of the matrix, is similar for the ceramic body of the Besztercebánya/Banská Bystrica and Csábrág/Čabrad’ tiles, but the characteristic non-plastic components in the bodies are different. From a macroscopic, petrographic, and chemical point of view, the ceramic bodies from the Hungarian sites (Salgó, Szécsény, Eger) and from Fülek/Fil'akovo form an independent group. We conclude that the differences in the body characteristics result from the different raw materials and paste preparation techniques, and thus from different workshop practices.

Although the ceramic bodies are different, the production technology of the lead glazes (mixing lead compound with silica and possibly applying to an unfired ceramic body) is similar for the Besztercebánya/Banská Bystrica, the Hungarian, and the Fülek/Fil'akovo tiles. The simplest and cheapest green glaze, coloured with copper, was usually applied to the stove tiles. The Besztercebánya/Banská Bystrica tiles are an exception, as lead antimonate is present in the green glaze of most of the tiles. This important technological difference indicates that the master(s) of the Besztercebánya/ Banská Bystrica tiles may have known that a small amount of lead antimonate colourant in the glaze generates a yellowish tone. Alternatively, they may have had access to another raw 


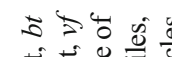

苛言尽:

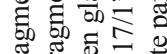

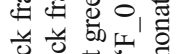

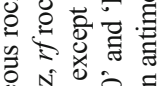

串

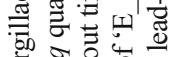

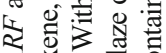

की

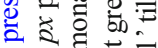

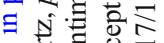

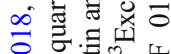

กे.

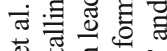

:

究

o

एँ

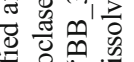

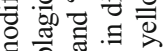

5 on

论 흘

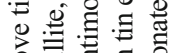

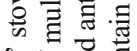

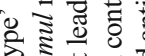

o 言焉

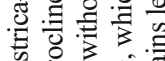

家.

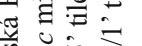

$\cong$ in

लำ 00

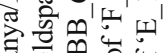

त ए

记

क कo

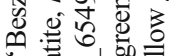

क्ष

究

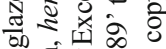

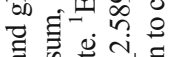

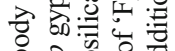

的数

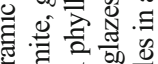

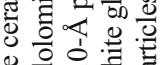

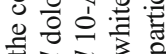

낭 횽 취

के ई

을 옹

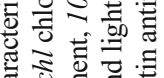

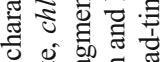

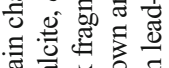

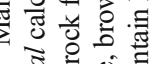

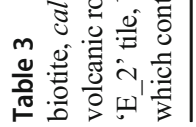

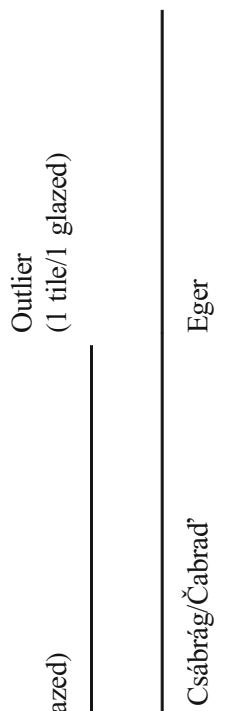

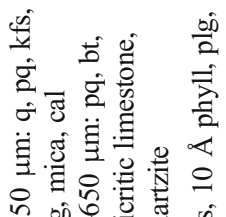

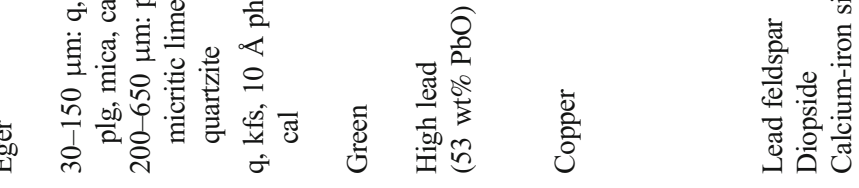

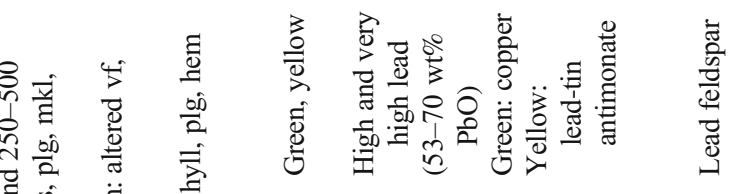

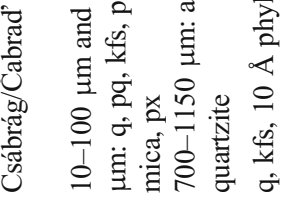

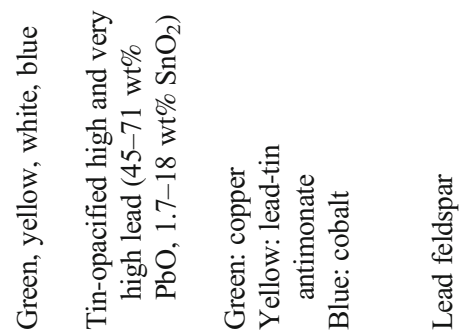

웅 리

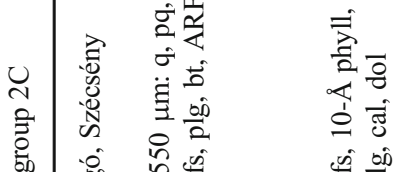

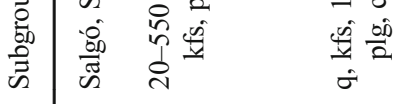

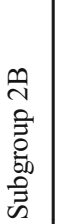

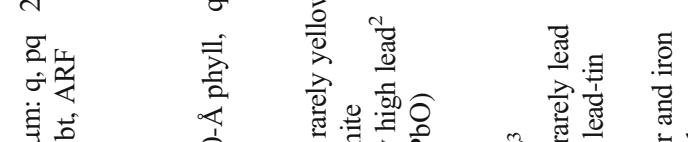

离

i⿱

选

产 $\bar{\Xi}$

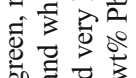

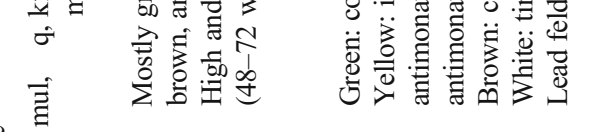

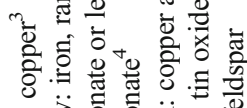

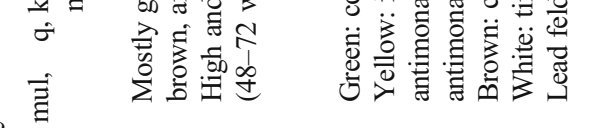

$\underline{4}$

की

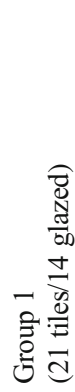

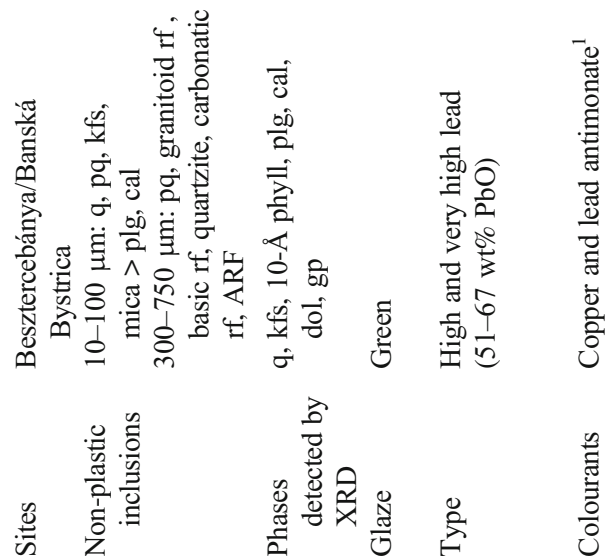

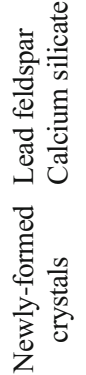


material that contained antimony. The few polychrome tiles were connected to the castles of Eger and Fülek/Filakovo.

One outlier tile from Eger (green-glazed grape tendrils 7) was similar to Besztercebánya/Banská Bystrica tiles (e.g. similar non-plastic components in the body); its green glaze did not contain lead antimonate.

In contrast, the polychrome (tin-opacified high lead) and the monochrome (high lead) glazes of the stove tiles at the castle of Csábrág/Čabrad' were produced by different technologies, indicating that the master(s) was/were highly skilled and used the exact materials that they needed. To achieve a more vivid and opaque green, yellow, white, or blue glaze on the polychrome tiles, tin oxide or lead-tin antimonate were added as a colourant and opacifier. The simpler method of a traditional lead glaze was used for the monochrome green glazes, as opacity was not necessary to achieve the desired colour and tone.

Based on the geology of the Banská Bystrica region, the raw materials for the glazes (such as lead, copper, tin, and cobalt) are both available in the vicinity. Written sources, however, only mention lead and copper mines in the region during the researched time period. There is only indirect evidence for tin mines in the Early Middle Ages, and no evidence for them in the Late Middle Ages (fifteenth-sixteenth centuries) (Batizi 2018). The origin of cobalt is also uncertain, as the presence of cobalt in the region does not imply that the masters used local cobalt sources at that time. From the thirteenth to the eighteenth centuries, the utilised cobalt sources in Central Europe were located in the Erzgebirge region (Gratuze et al. 1996; Zucchiatti et al. 2006), which may be the source of the cobalt used in the glaze.

The complex craft organisation of tile production in the medieval Carpathian Basin is still unknown. Technological features indicate that each workshop had its own clay source in the vicinity, as well as its own recipe and methodology for the glaze. Exchange of knowledge may have occurred between the workshops producing the tiles from Besztercebánya/ Banská Bystrica (group 1), from the Hungarian sites and from Fülek/Fil'akovo (group 2). Due to the geographical proximity of the Hungarian sites with Fülek/Fil'akovo, the similarities in the glazing technologies may indicate that one workshop manufactured the tiles. Furthermore, the considerable similarities in the size and motifs of these tiles suggest technological transfer between the workshops, possibly through the exchange of negatives (Györkös et al. in press).

Some tiles from Besztercebánya/Banská Bystrica ('BB_6549', 'BB_6555'), Eger ('E_2', 'E_10'), and Fülek/ Fil'akovo ('F_2.589', 'F_012', 'F_017/1') are exceptions. These exceptions are primarily due to (i) the presence of tin in dissolved or discrete form and/or lead-tin antimonate in the high-lead glazes of Eger and Fülek/Fil'akovo tiles, or (ii) due to the absence of lead antimonate in the green glaze of two Besztercebánya/Banská Bystrica tiles. Possible explanations for these findings include the intent of the master, the preparation of glazes in different proportions, a shortage of raw materials, additional tile pieces added during repair of the stove, tiles produced for the margin or for the back of the stove, tiles forming part of another stove, tiles as archetypes for the masters, tiles produced by different masters of the same workshop, or tiles produced by different workshops.

\section{Conclusion}

As a result of the first systematic study on the raw materials and production technologies of the ceramic body and the glaze, the peculiar late medieval 'Besztercebánya/Banská Bystrica-type' stove tiles were classified into three primary groups: (1) tiles from Besztercebánya/Banská Bystrica, (2) tiles from the Hungarian sites and Fülek/Fil'akovo, and (3) tiles from the castle of Csábrág/Čabrad'. Tiles found at several archaeological sites in the northern part of the Carpathian Basin, and its surrounding areas were produced in at least three different workshops in the region, from where they were distributed to customers. The exchange of negatives is potential evidence that knowledge was shared between the workshops.

The glazing technique showed that the technological expertise of the master(s) who produced the polychrome tiles from Csábrág/Čabrad' was higher than that of the master(s) who produced the tiles from other sites, pointing towards the Italian maiolica glazing tradition (e.g. use of tin oxide as an opacifier in the glaze). Expensive raw materials, such as tin and cobalt, in the polychrome tile glazes suggest a rich, high-status customer. At that time, the Csábrág/Čabrad' castle was likely owned by Archbishop Tamás Bakócz, who may have also been the customer of the decorative stove (Rakonczay 2018, 2020).

The location of the workshops as well as their existence through time is still uncertain. Previous archaeological and art historical studies suggest that the first workshop(s) may have been situated in Besztercebánya/Banská Bystrica, from where the illustrations later spread throughout the region. Tiles became higher in quality, more colourful, and more expensive, perhaps due to greater skill or at the behest of wealthy customers. Alternatively, the first workshop that produces highquality polychrome stove tiles may have been located in around Csábrág/Čabrad'. Thus, due to the transmission of the new fashion, the raw materials and production technology of the tiles simplified with time. Examination of the 'Besztercebánya/Banská Bystrica-type' stove tiles from other archaeological sites and new discoveries of future excavations may refine these theories and confirm the exchange of technological knowledge between workshops.

Supplementary Information The online version of this article (https:// doi.org/10.1007/s12520-020-01221-z) contains supplementary material, which is available to authorized users. 
Acknowledgements The Department of Mineral Sciences of the Smithsonian Institution (USA) is acknowledged for providing glass standards (Smithsonian Microbeam Standards) for the EDS analysis. We gratefully thank Emese Balogh-László, Rita Rakonczay, and Anna Anderko (Department of Hungarian Medieval and Early Modern Archaeology, Eötvös Loránd University in Budapest, Hungary), as well as Diána Radványi and Kornélia Hajtó (Museum of Applied Arts, Budapest, Hungary) for providing the stove tiles and the archaeological background. For the art historical background, we are grateful for Emese Mezei's expertise. We would also like to thank Judit Szabó (Geographical Institute, RCAES, Budapest, Hungary) for her contribution. We are grateful to Ariana Gugora (Institute for Geological and Geochemical Research, RCAES, Budapest, Hungary) for proofreading.

Funding Open access funding provided by ELKH Research Centre for Astronomy and Earth Sciences. This research was supported by the European Union and the State of Hungary, and co-financed by the European Regional Development Fund in the project of GINOP-2.3.215-2016-00009 'ICER'. László E. Aradi's work was granted by the ELTE Institutional Excellence Program (1783-3/2018/FEKUTSRAT), which is supported by the Hungarian Ministry of Human Capacities.

Open Access This article is licensed under a Creative Commons Attribution 4.0 International License, which permits use, sharing, adaptation, distribution and reproduction in any medium or format, as long as you give appropriate credit to the original author(s) and the source, provide a link to the Creative Commons licence, and indicate if changes were made. The images or other third party material in this article are included in the article's Creative Commons licence, unless indicated otherwise in a credit line to the material. If material is not included in the article's Creative Commons licence and your intended use is not permitted by statutory regulation or exceeds the permitted use, you will need to obtain permission directly from the copyright holder. To view a copy of this licence, visit http://creativecommons.org/licenses/by/4.0/.

\section{References}

Adams AE, Mackenzie WS, Guilford C (1984) Atlas of sedimentary rocks under the microscope. Longman, Harlow

Adlington LW (2017) The Corning Archaeological Reference Glasses: new values for "old" compositions. Pap Inst Archaeol 27(1):1-8

Anderko A (in press) A „besztercebányai kör” kályhacsempéi Fülek várában (Stove tiles of the "Besztercebánya collection" in the castle of Fülek). In: Fiatal Középkoros Régészek 9. Konferenciájának Tanulmánykötete (Proceedings of the IX. Young Medieval Archaeologists Conference)

Balogh-László E, Györkös D, Bajnóczi B, Tóth M (2018) Besztercebányai jellegü kályhacsempék régészeti és archeometriai kutatása (Archaeological and archaeometric investigation of the Besztercebánya type stove tiles). In: Ringer I (ed.) Fiatal Középkoros Régészek 8. Konferenciájának Tanulmánykötete (Proceedings of the VIII. Young Medieval Archaeologists Conference). Sátoraljaújhely, pp 303-318

Batizi I (2018) Mining in medieval Hungary. In: Laszlovszky J, Nagy B, Szabó P, Vadas A (eds) The Economy of Medieval Hungary. Brill, Leiden, pp 166-181

Bauer D, Ben Amara A, Cantin N (in press) La céramique de poele du site Berg Armo (XVI s., Sainte-Marie-aux-Mines, France): matériaux et techniques de fabrication. ArcheoSciences - Revue d'Archéométrie
Bodnár K (1988) Kályhacsempék Nógrád megyéből I. Szécsény mezőváros XV-XVI. századi kályhacsempéi (Stove tiles from Nógrád county). Nógrád Megyei Múzeumok Évkönyve 14:9-25

Chiarantini L, Gallo F, Rimondi V, Benvenuti M, Costagliola P, Dini A (2015) Early Renaissance production recipes for Naples yellow pigment: a mineralogical and lead isotope study of Italian majolica from Montelupo (Florence). Archaeometry 57:879-896

Cultrone G, Rodriguez-Navarro C, Sebastian E, Cazalla O, De La Torre MJ (2001) Carbonate and silicate phase reactions during ceramic firing. Eur J Mineral 13:621-634

Cultrone G, Molina E, Arizzi A (2014) The combined use of petrographic, chemical and physical techniques to define the technological features of Iberian ceramics from the Canto Tortoso area (Granada, Spain). Ceram Int 40:10803-10816

de Langhe K, Vekemans B, de Clercq W, Vincze L, Vandenabeele P (2015) Heating the house. An archaeological and archaeometrical investigation into the tile-stoves of late-medieval Flanders, Belgium (14-17th centuries). Post-Medieval Archaeol 49(2):291-312

Dik J, Hermens E, Peschar R, Schenk H (2005) Early production recipes for lead antimonate yellow in Italian art. Archaeometry 47:593-607

Ernée M, Hanykýř V, Maryška M (2004) Výsledky přírodovědných analýz gotických kamnových kachlů z Českého Krumlova (Ergebnisse naturwissenschaftlicher Analysen der gotischen Ofenkacheln aus Český Krumlov). Památky Archeologické XCV: 175-222

Feld I, Balogh-László E, Tóth B (2013) Régészeti kutatások a salgói várban (Archäologische Forschungen in der Burg Salgó). Archaeológiai Értesítő 138:239-264

Gratuze B, Soulier I, Blet M, Vallauri L (1996) De l' origine du cobalt: du verre á la céramique. Revue d' Archéométrie 20:77-94

Gruia A-M (2007) Sex on the stove. A fifteen-century tile from Banská Bystrica. Studia Patzinaka 4:85-122

Gruia A-M (2013) Religious representations on stove tiles from the medieval kingdom of Hungary. Editura Mega, Cluj-Napoca

Györkös D, Bajnóczi B, Gy Szakmány, Balogh-László E, Szabó M, Tóth M (2018) A besztercebányai (Banská Bystrica) típusú kályhacsempék archeometriai kutatásának előzetes eredményei (Preliminary results of the archaeometric investigation on the socalled Besztercebánya/Banská Bystrica type stove tiles). Archeometriai Mühely 15(1):45-56

Györkös D, Bajnóczi B, Szakmány Gy, Balogh-László E, Szabó M, Tóth $M$ (in press) Petrographic and XRD analyses of the ceramic body of medieval Besztercebánya/Banská Bystrica type stove tiles. ArcheoSciences - Revue d'Archéométrie

Heimann RB (2010) Classic and advanced ceramics. Wiley-VCH Verlag GMBH \& Co., Weinheim

Hoššo J (2005) Kachliarstvo v stredovekom meste Bratislava a v bratislavskom regióne. In: Chovanec J (ed.) Gotické a renesančné kaclice v Karpatoch. Trebišov, pp 131-148

Kocsis E (2018) A kályha története a középkori és kora újkori Magyarországon (History of stoves in the late Medieval and early Modern period of Hungary). In: Kiss V, Spekner E, Végh A (ed.) Szívmelengető középkor. Kályhák és kályhacsempék a középkori Magyarországon, 14-16. század: kiállítási katalógus (Heartwarming Middle Ages. Stoves and Stove-tiles in Medieval Hungary, 14th16th century: exhibition catalogue). Budapesti Történeti Múzeum, Budapest, pp 25-31

Kocsis E, Laszlovszky J, Sabján T, Takács M, Tomka G (2003) Medieval material culture-medieval archaeology. In: Visy Zs (ed) Hungarian archaeology at the turn of the Millennium. Ministry of National Cultural Heritage, Budapest, pp 397-405

Kreiter A, Pánczél P (2016) Petrographic analysis of ceramics from the Ottoman-Turkish Fort at Barcs (Hungary) and the neighboring settlements. In: Kovács Gy, Zatykó Cs (eds.) "per sylvam et per lacus nimios". The Medieval and Ottoman period in Southern 
Transdanubia, Southwest Hungary: the contribution of the natural sciences. Institute of Archaeology RCH HAS, Budapest, pp 95-132

Kvietok M, Mácelová M (2013) Krása kachlíc (Beautiful tiles). Katalóg výstavy. Stredoslovenské múzeum, Banská Bystrica

Lafuente B, Downs R, Yang H, Stone N (2015) The power of databases: the RRUFF project. In: Armbruster T, Danisi RM (eds) Highlights in mineralogical crystallography. De Gruyter, Berlin, pp 1-30

Lahlil S, Biron I, Galoisy L, Morin G (2008) Rediscovering ancient glass technologies through the examination of opacifier crystals. Appl Phys A92:109-116

László E (2012) A salgói vár kályhaszem-és kályhacsempe elemei (Stove Eye and Tile Finds in the Fortress of Salgó). Neograd 2011 Nógrád Megyei Múzeumok Évkönyve 35:179-206

Lightbown R, Caiger-Smith A (2007) Cipriano Piccolpasso's The Three Books of the Potter's Art (I tre libri dell'arte del vasaio): a facsimile of the manuscript in the Victoria and Albert Museum, London, 2nd edition, Editions La Revue de la céramique et du verre. Vendil-leViel, France

Mácelová M (2005) Ikonografia gotických kachlíc z banskobystrickej radnice. In: Chovanec J (ed.) Gotické a renesančné kaclice v Karpatoch. Trebišov, pp 205-216

Mácelová M (2006) Atribútý svätca na gotických kachliciach stredoslovenskej banskej oblasti. In Kožiak R, Nemeš J (ed.) Svätec a jeho funkcie v spoločnosti I. Bratislava, pp 369-382

Mácelová M (2009) Nepublikovaný súbor neskorogotických kachlíc z Dolnej ulice v Banskej Bystrici. Archaeologia Historica 34:399-443

Maggetti M (1982) Phase analysis and its significance for technology and origin. In: Olin JS, Franklin AD (eds) Archaeological Ceramic. Smithsonian Institution, Washington, pp 121-133

Maggetti M (2012) Technology and provenancing of French faience. In: Herrero JM, Vendrell M (eds) International Seminar on Archaeometry and cultural heritage: the contribution of mineralogy, Bilbao, 27 de Junio 2012. Seminarios del la Sociedad Española de Mineralogía 09:41-64

Maggetti M (2016) Technologische Analyse der Scharffeuerfarben dreier Kachelöfen von der Reichengasse 59 in Freiburg/Schweiz. Bulletin de la Société Fribourgeoise des Sciences Naturelles=Bulletin der Naturforschenden Gesellschaft Freiburg 105:91-119

Maggetti M (2019) Der Rüdlinger Kachelofen - Naturwissenschaftliche Analyse des 1681/1682 datierten Ofens des Winterthurer Hafners Abraham Pfau. Keramik-Freunde der Schweiz Mitteilungsblatt 133: $15-66$

Maggetti M, Serneels V (2016) Chemische und technologische Analyse einiger Fayencekacheln eines JOHANN BAPTIST NUOFFER zugeschriebenen Kachelofens von ca. 1780-85. Bulletin de la Société Fribourgeoise des Sciences Naturelles=Bulletin der Naturforschenden Gesellschaft Freiburg 105:32-90

Maggetti M, Neururer CH, Rosen J (2009) Antimonate opaque glaze colours from the faience manufacture of Le Bois d'Épense (19th century, Northeastern France). Archaeometry 51/5:791-807

Maggetti M, Galetti G, Torche-Julmy M-T (2014) Analyses scientifiques de quelques catelles de poêles fribourgeois du XVIIIe s. Bulletin de la Société Fribourgeoise des Sciences Naturelles=Bulletin der Naturforschenden Gesellschaft Freiburg 103:59-82

Mason RB, Tite MS (1997) The beginnings of tin-opacification of pottery glazes. Archaeometry 39:41-58

Mass JL, Stone RE, Wypyski MT (1996) An investigation of the antimony-containing minerals used by the Romans to prepare opaque colored glasses. Mater Res Soc Proc 462:193-206

Mass J, Stone R, Wypyski M (1998) The mineralogical and metallurgical origins of Roman opaque colored glasses. In: McCray P and Kingery WD (eds.) The prehistory and history of glassmaking technology, Ceramics and Civilisation VIII, The American Ceramics Society, Westerville, Ohio, pp 121-144
Mass JL, Wypyski MT, Stone RE (2002) Malkata and Lisht glassmaking technologies: towards a specific link between second millennium BC metallurgists and glassmakers. Archaeometry 44:67-82

Matin M (2019) Tin-based opacifiers in archaeological glass and ceramic glazes: a review and new perspectives. Archaeol Anthropol Sci 11: 1155-1167

Matin M, Tite M, Watson O (2018) On the origins of tin-opacified ceramic glazes: new evidence from early Islamic Egypt, the Levant, Mesopotamia, Iran, and Central Asia. J Archaeol Sci 97:42-66

Mezei E (2013) Az Ebner-csempék. Egy késő középkori kályháról (The Ebner stove tiles). In: J Újváry Zs (ed.) Győzteseink szárnypróbálásai. A PPKE BTK bölcsészhallgatóinak győztes dolgozatai a XXXI. OTDK HUMÁN Szekcióban. Pázmány Péter Katolikus Egyetem Bölcsészet- és Társadalomtudományi Kar, Piliscsaba, pp 271-300

Mezei E (2016) Késő középkori kályhacsempék Besztercebányáról (Late Medieval stove tiles from Besztercebánya). In: Simonyi E, Tomka G (ed.) „A cserép igazat mond, ha helyette nem mi akarunk beszélni”. Regionalitás a középkori és kora újkori kerámiában. A Magyar Nemzeti Múzeumban 2013. január 9-11. között rendezett konferencia előadásai. Opuscula Hungarica IX, Magyar Nemzeti Múzeum, Budapest, pp 313-318.

Molera J, Pradell T, Salvado N, Vendrell-Saz M (2001) Interactions between clay bodies and lead glazes. J Am Ceram Soc 84:1120-1128

Molina G, Odin GP, Pradell T, Shortland AJ, Tite MS (2014) Production technology and replication of lead antimonate yellow glass from New Kingdom Egypt and the Roman Empire. J Archaeol Sci 41: 171-184

Mordovin M (2015) New results of the excavations at the Saint James' Pauline friary and at the Castle Čabrad'. Dissertationes Archaeol Ser 3(3):269-283

Palamara E, Zacharias N, Xanthopoulou M, Kasztovszky Zs, Kovács I, Palles D, Kamitsos EI (2016) Technology issues of Byzantine glazed pottery from Corinth, Greece. Microchem J 129:137-150

Parádi N (1984) A besztercebányai kályhacsempék lelőhelyéről (Über die Fundstelle der Ofenkacheln von Besztercebánya (Banská Bystrica)). Folia Archeol 35:175-184

PCRG (2010) The study of prehistoric pottery: general policies and guidelines for analysis and publication. Prehistoric Ceramics Research Group: Occasional Papers Nos 1 and 2. 3rd edition revised.

Pradell T, Molina G, Molera J, Pla J, Labrador A (2013) The use of micro-XRD for the study of glaze color decorations. Appl Phys A111:121-127

Quinn PS (2013) Ceramic petrography. The interpretation of archaeological pottery \& related artefacts in thin section. Archaeopress, Oxford

Rakonczay R (2018) “Der Kachelofen des Erzbischofs". Ofenkacheln aus der Burg Čabrad' (Slowakei) und der Kachelkreis von Neusohl um 1500. Burgen und Schlösser 4:216-225

Rakonczay R (2020) "The Stove of the Archbishop". Figural stove tiles from the Castle of Csábrág (Čabrad'). Hung Archaeol E-J 9/2:3848. https://doi.org/10.36338/ha.2020.2.1

Rodriguez-Navarro C, Cultrone G, Sanchez-Navas A, Sebastian E (2003) TEM study of mullite growth after muscovite breakdown. Am Mineral 88:713-724

Rosi F, Manuali V, Grygar T, Bezdicka P, Brunetti BG, Sgamellotti A, Burgio L, Seccaroni C, Miliani C (2011) Raman scattering features of lead pyroantimonate compounds: implications for the noninvasive identification of yellow pigments on ancient ceramics. Part II. In situ characterisation of Renaissance plates by portable micro-Raman and XRF studies. J Raman Spectrosc 42:407-414

Roth-Heege E (2018) Az európai cserépkályhák korai története (Early history of the stoves in Europe). In: Kiss V, Spekner E, Végh A (ed.) Szívmelengető középkor. Kályhák és kályhacsempék a középkori Magyarországon, 14-16. század: kiállítási katalógus (Heartwarming Middle Ages. Stoves and Stove-tiles in Medieval 
Hungary, 14th-16th century: exhibition catalogue). Budapesti Történeti Múzeum, Budapest, pp 33-39

S. Cserey É (1974) Adatok a Besztercebányai (Banská Bystrica) kályhacsempékhez ( $\mathrm{Zu}$ den Ofenkacheln von Besztercebánya (Banská Bystrica)). Folia Archaeologica 25:205-217

Salinas E, Pradell T, Molera J (2019a) Glaze production at an early Islamic workshop in al-Andalus. Archaeol Anthropol Sci 11: 2201-2213

Salinas E, Pradell T, Matin M, Tite MS (2019b) From tin- to antimonybased yellow opacifiers in the early Islamic Egyptian glazes: regional influences and ruling dynasties. J Archaeol Sci Rep 26:101923

Sandalinas C, Ruiz-Moreno S (2004) Lead-tin-antimony yellow: historical manufacture, molecular characterization and identification in seventeenth-century Italian paintings. Stud Conserv 49(1):41-52

Sandalinas C, Ruiz-Moreno S, López-Gil A, Miralles J (2006) Experimental confirmation by Raman spectroscopy of a $\mathrm{Pb}-\mathrm{Sn}-\mathrm{Sb}$ triple oxide yellow pigment in sixteenth-century Italian pottery. J Raman Spectrosc 37:1146-1153

Schairer JF, Bowen NL (1947) Melting relations in the systems $\mathrm{Na}_{2} \mathrm{O}$ $\mathrm{Al}_{2} \mathrm{O}_{3}-\mathrm{SiO}_{2}$ and $\mathrm{K}_{2} \mathrm{O}-\mathrm{Al}_{2} \mathrm{O}_{3}-\mathrm{SiO}_{2}$. Am J Sci 245:193-204

Shortland AJ (2000) Vitreous materials at Amarna: the production of glass and faience in 18th Dynasty Egypt. British Archaeological Reports International Series, 827, Archaeopress, Oxford

Shortland AJ (2002) The use and origin of antimonate colorants in Early Egyptian glass. Archaeometry 44(4):517-530

Ting C, Lichtenberger A, Raja R (2019) The technology and production of glazed ceramics from Middle Islamic Jerash, Jordan. Archaeometry 61(6):1296-1312
Tite MS (2009) The production technology of Italian maiolica: a reassessment. J Archaeol Sci 36:2065-2080

Tite MS, Freestone I, Mason R, Molera J, Vendrell-Saz M, Wood N (1998) Lead glazes in antiquity-methods of production and reasons for use. Archaeometry 40:241-260

Tite M, Pradell T, Shortland A (2008) Discovery, production and use of tin-based opacifiers in glasses, enamels and glazes from the Late Iron Age onwards: a reassessment. Archaeometry 50:67-84

Vecstaudža J, Jakovḷevs D, Bērziṇa-Cimdina L, Stikāne V (2013) XRD and SEM studies of archaeological stove tile ceramics of Turaida Castle. Mater Sci Appl Chem 29:40-45

Wainwright INM, Taylor JM, Harley RD (1986) Lead antimonate yellow. In: Feller RL (ed.) Artists' pigments. A Handbook of their History and Characteristics, Vol 1. Cambridge University Press, Cambridge and National Gallery of Art, Washington, pp 219-254.

Walton WS, Tite MS (2010) Production technology of Roman leadglazed pottery and its continuance into Late Antiquity. Archaeometry 52:733-759

Zucchiatti A, Bouquillon A, Katona I, D'Alessandro A (2006) The 'Della Robbia Blue': a case study for the use of cobalt pigments in ceramics during the Italian Renaissance. Archaeometry 48(1):131-152

Publisher's note Springer Nature remains neutral with regard to jurisdictional claims in published maps and institutional affiliations. 